\title{
Notes on Seismic Source Models in Elastostatics
}

TECHNICAL Notes

\author{
Deyu Ming* \\ Department of Statistical Science \\ University College London \\ London, UK
}

July 29, 2020

\begin{abstract}
Seismic source models in elastostatics lay the foundations for the popular earthquake deformation formula derived in Okada (1992), which forms key components for many studies in earthquake displacement simulations, earthquake source inversions and ground-motion predictions. However, despite of their importance, we found that the assumptions and proofs of some important theorems about the topic are incomplete in the literature, causing difficulties for beginners to understand the theoretical basis of Okada (1992) and its extensions. This note fills these gaps, and conveys the mathematics of relevant concepts in a consistent (in terms of notations) and rigorous (in terms of assumptions and deviations) manner. The note could be used as a complement to the more general introduction to the quantitative seismology provided in Moczo (2006) and Aki (2009).

This note is a non-peer reviewed EarthArXiv preprint. These notes are meant for a general audience who want to understand in depth the mathematical proofs of some important theorems involved in seismic source models in elastostatics and their implicit assumptions. The main sources of the material are Moczo (2006) and Aki (2009). Please consult listed references for more details of the topics discussed. Please feel free to contact the author for any feedback.
\end{abstract}

Keywords Constitutive equation, equation of motion, Green's tensor, principle of superposition, uniqueness theorems, reciprocity theorem, representation theorem

\section{Displacement}

The displacement can be defined as the movement of individual points from their original positions due to various external loads. The standard unit of displacement is meter $(m)$. Mathematically, assume there is a Cartesian coordinate system in a continuum and the position vector $\mathbf{r}$ is denoted by

$$
\mathbf{r}=(x, y, z)^{\top}=x \mathbf{e}^{1}+y \mathbf{e}^{2}+z \mathbf{e}^{3},
$$

where $\mathbf{e}^{1}, \mathbf{e}^{2}$ and $\mathbf{e}^{3}$ are unit vectors in $x, y$ and $z$ directions respectively. Suppose there is a point at position $\mathbf{r}$, then the displacement of the point is given by

$$
\mathbf{u}(\mathbf{r})=\left(u_{x}(\mathbf{r}), u_{y}(\mathbf{r}), u_{z}(\mathbf{r})\right)^{\top}=u_{x}(\mathbf{r}) \mathbf{e}^{1}+u_{y}(\mathbf{r}) \mathbf{e}^{2}+u_{z}(\mathbf{r}) \mathbf{e}^{3} .
$$

${ }^{*}$ Corresponding author: deyu.ming.16@ucl.ac.uk. 
The displacement $\mathbf{u}(\mathbf{r})$ of any arbitrary point at the position $\mathbf{r}=(x, y, z)^{\top}$ can be treated as a vector-valued function, $\mathbf{u}(\mathbf{r}): \mathbb{R}^{3} \rightarrow \mathbb{R}^{3}$. In another word, $\mathbf{u}(\mathbf{r})$ is a function of position vector $\mathbf{r}=(x, y, z)^{\top}$ and its output is a three-element distance vector, measuring the displacement of a point from its original position $\mathbf{r}$.

Remark The displacement of a point also depends on time. However, some work such as Robinson \& Benites (1996), Zhou et al. (2006) assume that the model for displacement is time-independent.

\section{Strain Tensor}

The displacements of points within a particle will change the particle in four basic forms:

- Translation;

- Rotation;

- Distortion;

- Dilation.

The translation of a particle is due to the parallel translations of points within it. The distortion and dilation is the change in shape and volume of the particle respectively.

In order to examine how points within a particle move relative to each other due to external loads, we consider a Cartesian coordinate in a continuum and a point $P$ at position $\mathbf{r}_{0}=\left(x_{0}, y_{0}, z_{0}\right)^{\top}$. Assume another point $Q$, a small distance (donated by the distance vector $\mathbf{d}=\left(d_{x}, d_{y}, d_{z}\right)^{\top}$ ) away from point $P$, at position $\mathbf{r}=(x, y, z)^{\top}$. The displacements of points $P$ and $Q$ due to external loads are donated by $\mathbf{u}\left(\mathbf{r}_{0}\right)$ and $\mathbf{u}(\mathbf{r})$ respectively. By applying Taylor expansion to $\mathbf{u}(\mathbf{r})$ at $\mathbf{r}_{0}$, we can obtain

$$
\mathbf{u}(\mathbf{r})=\mathbf{u}\left(\mathbf{r}_{0}\right)+\mathbf{J d}
$$

where

$$
\mathbf{J}=\left[\begin{array}{lll}
\frac{\partial u_{x}\left(\mathbf{r}_{0}\right)}{\partial x} & \frac{\partial u_{x}\left(\mathbf{r}_{0}\right)}{\partial y} & \frac{\partial u_{x}\left(\mathbf{r}_{0}\right)}{\partial z} \\
\frac{\partial u_{y}\left(\mathbf{r}_{0}\right)}{\partial x} & \frac{\partial u_{y}\left(\mathbf{r}_{0}\right)}{\partial y} & \frac{\partial u_{y}\left(\mathbf{r}_{0}\right)}{\partial z} \\
\frac{\partial u_{z}\left(\mathbf{r}_{0}\right)}{\partial x} & \frac{\partial u_{z}\left(\mathbf{r}_{0}\right)}{\partial y} & \frac{\partial u_{z}\left(\mathbf{r}_{0}\right)}{\partial z}
\end{array}\right]
$$

the Jacobian matrix of $\mathbf{u}(\mathbf{r})$ realised at $\mathbf{r}=\mathbf{r}_{0}$. According to Shearer (2009), higher order terms of partials are ignored during the expansion in the sense that Earth strains are so small that the approximation is valid. $\mathbf{J}$ can be further decomposed into a symmetric part $\epsilon$ and an anti-symmetric part $\Omega$ :

$$
\mathbf{J}=\boldsymbol{\epsilon}+\boldsymbol{\Omega},
$$

where $\epsilon$ is the strain tensor given by

$$
\boldsymbol{\epsilon}=\left[\begin{array}{ccc}
\frac{\partial u_{x}\left(\mathbf{r}_{0}\right)}{\partial x} & \frac{1}{2}\left(\frac{\partial u_{x}\left(\mathbf{r}_{0}\right)}{\partial y}+\frac{\partial u_{y}\left(\mathbf{r}_{0}\right)}{\partial x}\right) & \frac{1}{2}\left(\frac{\partial u_{x}\left(\mathbf{r}_{0}\right)}{\partial z}+\frac{\partial u_{z}\left(\mathbf{r}_{0}\right)}{\partial x}\right) \\
\frac{1}{2}\left(\frac{\partial u_{y}\left(\mathbf{r}_{0}\right)}{\partial x}+\frac{\partial u_{x}\left(\mathbf{r}_{0}\right)}{\partial y}\right) & \frac{\partial u_{y}\left(\mathbf{r}_{0}\right)}{\partial y} & \frac{1}{2}\left(\frac{\partial u_{y}\left(\mathbf{r}_{0}\right)}{\partial z}+\frac{\partial u_{z}\left(\mathbf{r}_{0}\right)}{\partial y}\right) \\
\frac{1}{2}\left(\frac{\partial u_{z}\left(\mathbf{r}_{0}\right.}{\partial x}+\frac{\partial u_{x}\left(\mathbf{r}_{0}\right)}{\partial z}\right) & \frac{1}{2}\left(\frac{\partial u_{z}\left(\mathbf{r}_{0}\right)}{\partial y}+\frac{\partial u_{y}\left(\mathbf{r}_{0}\right)}{\partial z}\right) &
\end{array}\right]
$$

and $\Omega$ is the rotation tensor given by

$$
\boldsymbol{\Omega}=\left[\begin{array}{ccc}
0 & \frac{1}{2}\left(\frac{\partial u_{x}\left(\mathbf{r}_{0}\right)}{\partial y}-\frac{\partial u_{y}\left(\mathbf{r}_{0}\right)}{\partial x}\right) & \frac{1}{2}\left(\frac{\partial u_{x}\left(\mathbf{r}_{0}\right)}{\partial z}-\frac{\partial u_{z}\left(\mathbf{r}_{0}\right)}{\partial x}\right) \\
\frac{1}{2}\left(\frac{\partial u_{y}\left(\mathbf{r}_{0}\right)}{\partial x}-\frac{\partial u_{x}\left(\mathbf{r}_{0}\right)}{\partial y}\right) & 0 & \frac{1}{2}\left(\frac{\partial u_{y}\left(\mathbf{r}_{0}\right)}{\partial z}-\frac{\partial u_{z}\left(\mathbf{r}_{0}\right)}{\partial y}\right) \\
\frac{1}{2}\left(\frac{\partial u_{z}\left(\mathbf{r}_{0}\right)}{\partial x}-\frac{\partial u_{x}\left(\mathbf{r}_{0}\right)}{\partial z}\right) & \frac{1}{2}\left(\frac{\partial u_{z}\left(\mathbf{r}_{0}\right)}{\partial y}-\frac{\partial u_{y}\left(\mathbf{r}_{0}\right)}{\partial z}\right) & 0
\end{array}\right] .
$$

The off-diagonal components of strain tensor $\epsilon$ reflect the angle that each side of a particle rotates and thus control the distortion of the particle; The sum of diagonal components of $\epsilon$ (i.e., the trace of $\epsilon$ ) reflects the relative volume change of a particle and thus control the dilation of the particle; the rotation tensor $\Omega$ causes rigid rotation of a particle and thus control the rotation of the particle. More information on the effects of strain tensor and rotation tensor can be found in Shearer (2009).

Since strain tensor deals with shape and volume change of a particle, it is dimensionless. 


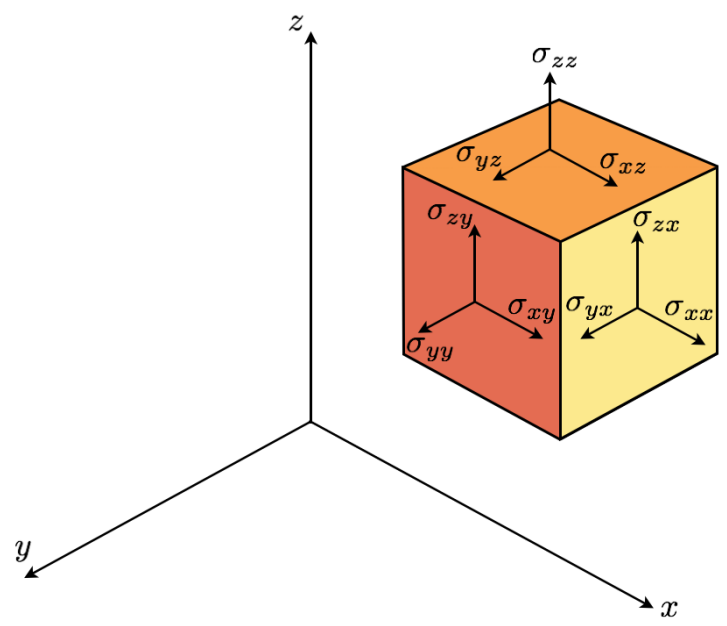

Figure 1: The geometry of coordinate stresses through a particle in a Cartesian coordinate.

\section{Stress}

Stress is the force per unit area and its standard unit is Newtons per square meter $\left(\mathrm{N} / \mathrm{m}^{2}\right)$ or Pascal $(\mathrm{Pa}: 1 \mathrm{~Pa}=$ $1 \mathrm{~N} / \mathrm{m}^{2}$ ). Assume there is a Cartesian coordinate system in a continuum and there is an infinitesimal plane of arbitrary orientation specified by its unit normal vector $\mathbf{n}=\left(n_{x}, n_{y}, n_{z}\right)^{\top}$. Then the stress vector across the plane specified by $\mathbf{n}$ is denoted by

$$
\mathbf{t}(\mathbf{n})=\left(t_{x}(\mathbf{n}), t_{y}(\mathbf{n}), t_{z}(\mathbf{n})\right)^{\top}=t_{x}(\mathbf{n}) \mathbf{e}^{1}+t_{y}(\mathbf{n}) \mathbf{e}^{2}+t_{z}(\mathbf{n}) \mathbf{e}^{3}
$$

where $\mathbf{e}^{1}, \mathbf{e}^{2}$ and $\mathbf{e}^{3}$ are unit vectors in $x, y$ and $z$ directions respectively. Gauchy's Stress Theorem (Irgens 2008) states that the stress vector $\mathbf{t}(\mathbf{n})$ is uniquely determined by $\mathbf{n}$ to the plane and the stress tensor $\boldsymbol{\sigma}$ :

$$
\mathbf{t}(\mathbf{n})=\boldsymbol{\sigma n}
$$

or in its complete form:

$$
\left[\begin{array}{c}
t_{x}(\mathbf{n}) \\
t_{y}(\mathbf{n}) \\
t_{z}(\mathbf{n})
\end{array}\right]=\left[\begin{array}{lll}
\sigma_{x x} & \sigma_{x y} & \sigma_{x z} \\
\sigma_{y x} & \sigma_{y y} & \sigma_{y z} \\
\sigma_{z x} & \sigma_{z y} & \sigma_{z z}
\end{array}\right]\left[\begin{array}{l}
n_{x} \\
n_{y} \\
n_{z}
\end{array}\right]
$$

There are 9 components in stress tensor $\sigma$ and each component represents a stress through different surface of a particle in different directions. These 9 components are called coordinate stresses (Irgens 2008) and are visualised in Figure 1 The coordinate stresses $\sigma_{x x}, \sigma_{y y}$ and $\sigma_{z z}$ are normal stresses while the rest 6 coordinate stresses are shear stresses. For each coordinate stress $\sigma_{i j}$ with $i, j \in\{x, y, z\}$, the first subscript $i$ identifies the direction of the stress and the second subscript $j$ refers to the direction of the unit normal vector to the surface on which the stress is exerted. The coordinate stresses can take both positive and negative values. We follow the sign rule given by Irgens (2008):

A coordinate stress is positive if

- it acts in the direction of a positive coordinate axis and the direction of the normal vector to the surface on which it exerted points to the positive direction of a coordinate axis.

- it acts in the direction of a negative coordinate axis and the direction of the normal vector to the surface on which it exerted points to the negative direction of a coordinate axis.

In other cases, a coordinate stress is negative. 
Since the continuum is in static equilibrium, coordinate shear stresses should be equal to prevent rotations of the particle on which they are exerted. Thus, $\sigma_{x y}=\sigma_{y x}, \sigma_{x z}=\sigma_{z x}$ and $\sigma_{y z}=\sigma_{z y}$, indicating the stress tensor is symmetric:

$$
\boldsymbol{\sigma}=\boldsymbol{\sigma}^{\top}=\left[\begin{array}{lll}
\sigma_{x x} & \sigma_{x y} & \sigma_{x z} \\
\sigma_{x y} & \sigma_{y y} & \sigma_{y z} \\
\sigma_{x z} & \sigma_{y z} & \sigma_{z z}
\end{array}\right] .
$$

In summary, there are only six independent elements in the stress tensor $\sigma$ and they are sufficient to fully describe the stress at a given position in the continuum. The normal stress $\tau$ exerted on a surface specified by the unit normal vector $\mathbf{n}$ can be calculated by

$$
\boldsymbol{\tau}=\left(\mathbf{n}^{\top} \boldsymbol{\sigma} \mathbf{n}\right) \mathbf{n} .
$$

The shear stress $\tilde{\boldsymbol{\tau}}$ in an arbitrary direction (specified by the unit direction vector $\mathbf{v}=\left(v_{x}, v_{y}, v_{z}\right)^{\top}$ ) on a surface specified by the unit normal vector $\mathbf{n}$ is given by

$$
\tilde{\boldsymbol{\tau}}=\left(\mathbf{v}^{\top} \boldsymbol{\sigma} \mathbf{n}\right) \mathbf{v} .
$$

\section{Transformation of Stress Tensor}

In the analysis of seismicity, changes of coordinate systems usually are involved. When the coordinate system differs, so does the stress tensor (see Figure 22. In the Cartesian coordinate system, if $x y z$-system is changed to $x^{\prime} y^{\prime} z^{\prime}$-system, then the stress tensor $\boldsymbol{\sigma}$ in the initial system is transformed into the stress tensor $\boldsymbol{\sigma}^{\prime}$ according to the transformation rule

$$
\boldsymbol{\sigma}^{\prime}=\mathbf{A} \boldsymbol{\sigma} \mathbf{A}^{\top}
$$

where

$$
\mathbf{A}=\left[\begin{array}{lll}
a_{x^{\prime} x} & a_{x^{\prime} y} & a_{x^{\prime} z} \\
a_{y^{\prime} x} & a_{y^{\prime} y} & a_{y^{\prime} z} \\
a_{z^{\prime} x} & a_{z^{\prime} y} & a_{z^{\prime} z}
\end{array}\right]
$$

is a rotation matrix representing the coordinate system changes. For each element $a_{i j}$ with $i \in\left\{x^{\prime}, y^{\prime}, z^{\prime}\right\}$ and $j \in\{x, y, z\}$, the first subscript $i$ identifies the axis in the $x^{\prime} y^{\prime} z^{\prime}$-system and the second subscript $j$ refers to the axis in the initial $x y z$-system. Each element $a_{i j}$ equals to the cosine of the angles between the $i$-axis in the $x^{\prime} y^{\prime} z^{\prime}$-system and the $j$ axis in the $x y z$-system.

\section{Geometric Law}

Given the position $\mathbf{r}_{0}$ of a particular point in a particle, the relationship between the displacement and strain tensor is found by equation (2). To simplify the notation, we rewrite the equation (2) in following form:

$$
\boldsymbol{\epsilon}=\frac{1}{2}\left(\nabla \mathbf{u}\left(\mathbf{r}_{0}\right)+\left(\nabla \mathbf{u}\left(\mathbf{r}_{0}\right)\right)^{\top}\right),
$$

where $\nabla \mathbf{u}\left(\mathbf{r}_{0}\right)$ is the gradient of displacement vector $\mathbf{u}\left(\mathbf{r}_{0}\right)$ operated by row and is equal to the Jacobian matrix $\mathbf{J}$.

\section{Constitutive Equation}

The relationship between stress and strain tensors in linear elastic media can be described by constitutive equation (generalised Hooke's law)

$$
\boldsymbol{\sigma}_{i j}=\sum_{k=1}^{3} \sum_{l=1}^{3} \mathbf{c}_{i j k l} \boldsymbol{\epsilon}_{k l},
$$

where $\boldsymbol{\sigma}_{i j}$ is the element on $i$-th row and $j$-th column of stress tensor; $\boldsymbol{\epsilon}_{k l}$ is the element on $k$-th row and $l$-th column of strain tensor; $\mathbf{c} \in \mathbb{R}^{9 \times 9}$ is the elastic tensor representing properties of the material. The subscript $i j k l$ determines locations of elements in elastic tensor $\mathbf{c}$ according to

$$
\text { row }=3(i-1)+j, \quad \text { column }=3(k-1)+l .
$$



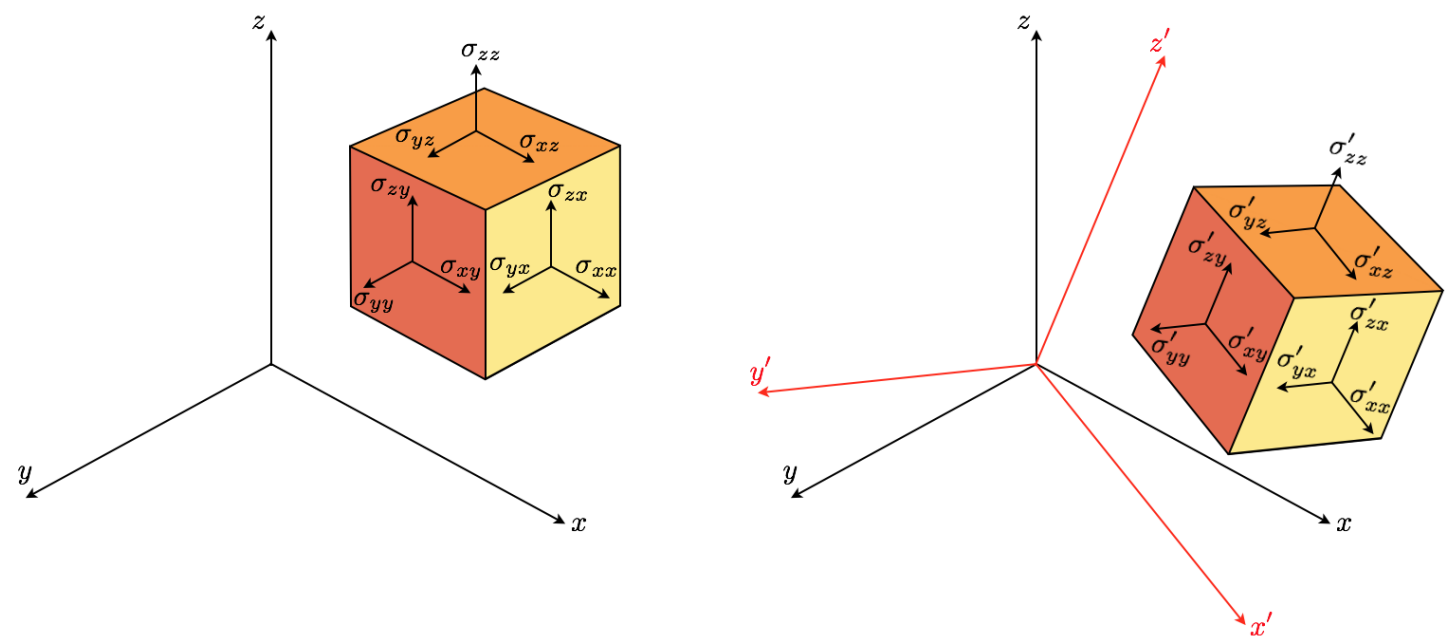

Figure 2: Transformation of the stress tensor in the Cartesian coordinate system $x y z$ to the one in the Cartesian coordinate system $x^{\prime} y^{\prime} z^{\prime}$.

Proposition 6.1 The elastic tensor c has the following symmetric properties:

$$
\boldsymbol{c}_{i j k l}=\boldsymbol{c}_{j i k l}, \quad \boldsymbol{c}_{i j k l}=\boldsymbol{c}_{i j l k} \quad \text { and } \quad \boldsymbol{c}_{i j k l}=\boldsymbol{c}_{k l i j} .
$$

Proof Due to the symmetry of stress tensor $\boldsymbol{\sigma}$, we have

$$
\boldsymbol{\sigma}_{i j}=\boldsymbol{\sigma}_{j i}
$$

Thus we have

$$
\begin{aligned}
\sum_{k=1}^{3} \sum_{l=1}^{3} \mathbf{c}_{i j k l} \boldsymbol{\epsilon}_{k l} & =\sum_{k=1}^{3} \sum_{l=1}^{3} \mathbf{c}_{j i k l} \boldsymbol{\epsilon}_{k l} \\
\sum_{k=1}^{3} \sum_{l=1}^{3}\left(\mathbf{c}_{i j k l}-\mathbf{c}_{j i k l}\right) \boldsymbol{\epsilon}_{k l} & =0 .
\end{aligned}
$$

Given that $\mathbf{e}$ is non-zero and arbitrary, we have

$$
\mathbf{c}_{i j k l}=\mathbf{c}_{j i k l} .
$$

Due to the symmetry of strain tensor e, we have

$$
\mathbf{e}_{i j}=\mathbf{e}_{j i}
$$

Thus, we obtain

$$
\begin{aligned}
\sum_{k=1}^{3} \sum_{l=1}^{3} \mathbf{c}_{i j k l} \boldsymbol{\epsilon}_{k l} & =\sum_{k=1}^{3} \sum_{l=1}^{3} \mathbf{c}_{i j l k} \boldsymbol{\epsilon}_{l k} \\
\sum_{k=1}^{3} \sum_{l=1}^{3} \mathbf{c}_{i j k l} \boldsymbol{\epsilon}_{k l} & =\sum_{k=1}^{3} \sum_{l=1}^{3} \mathbf{c}_{i j l k} \boldsymbol{\epsilon}_{k l} \\
\sum_{k=1}^{3} \sum_{l=1}^{3}\left(\mathbf{c}_{i j k l}-\mathbf{c}_{i j l k}\right) \boldsymbol{\epsilon}_{k l} & =0 .
\end{aligned}
$$

Given that $\mathbf{e}$ is non-zero and arbitrary, we get

$$
\mathbf{c}_{i j k l}=\mathbf{c}_{i j l k}
$$

To prove the last symmetry, we see the following equality:

$$
\sum_{i=1}^{3} \sum_{j=1}^{3} \sum_{k=1}^{3} \sum_{l=1}^{3} \mathbf{c}_{i j k l} \boldsymbol{\epsilon}_{i j} \boldsymbol{\epsilon}_{k l}=\sum_{k=1}^{3} \sum_{l=1}^{3} \sum_{i=1}^{3} \sum_{j=1}^{3} \mathbf{c}_{k l i j} \boldsymbol{\epsilon}_{k l} \boldsymbol{\epsilon}_{i j}
$$


The equality then gives:

$$
\sum_{i=1}^{3} \sum_{j=1}^{3} \sum_{k=1}^{3} \sum_{l=1}^{3}\left(\mathbf{c}_{i j k l}-\mathbf{c}_{k l i j}\right) \boldsymbol{\epsilon}_{i j} \boldsymbol{\epsilon}_{k l}=0 .
$$

Given that $\mathbf{e}$ is non-zero and arbitrary, we then have

$$
\mathbf{c}_{i j k l}=\mathbf{c}_{k l i j}
$$

Equation (6) says that the stress tensor only related to the strain tensor, indicating that the stress changes are due to changes of shape and volume of solids rather than particle translation and rotations. In seismology, the constitutive equation (6) is used to compute the stresses increased from the initial stress state with strain to be zero. We can also obtain the relation between the stress tensor $\boldsymbol{\sigma}$ and dispacement $\mathbf{u}(\mathbf{r})$ of a point at position $\mathbf{r}$ according to Equation (6):

Lemma 6.2 Assume $\mathbf{u}(\mathbf{r})$ is the displacement of any arbitrary point at position $\mathbf{r}=(x, y, z)^{\top}$ in an elastic medium, then the elements of stress tensor $\sigma$ can be expressed by:

$$
\boldsymbol{\sigma}_{i j}=\sum_{k=1}^{3} \sum_{l=1}^{3} \mathbf{c}_{i j k l} \frac{\partial \mathbf{u}_{k}(\mathbf{r})}{\partial \mathbf{r}_{l}}
$$

where $\mathbf{u}_{k}(\mathbf{r})$ is the $k$-th element of the displacement vector $\mathbf{u}(\mathbf{r})$ and $\mathbf{r}_{l}$ is the l-th element of the position vector $\mathbf{r}$.

Proof We can rewrite the Geometric Law (5) in element-wise form:

$$
\boldsymbol{\epsilon}_{k l}=\frac{1}{2}\left(\frac{\partial \mathbf{u}_{k}(\mathbf{r})}{\partial \mathbf{r}_{l}}+\frac{\partial \mathbf{u}_{l}(\mathbf{r})}{\partial \mathbf{r}_{k}}\right), \quad \forall k, l \in\{1,2,3\} .
$$

We then replace $\epsilon_{k l}$ in equation $(6)$ by equation $(8)$ and obtain:

$$
\begin{aligned}
\boldsymbol{\sigma}_{i j} & =\frac{1}{2} \sum_{k=1}^{3} \sum_{l=1}^{3} \mathbf{c}_{i j k l}\left(\frac{\partial \mathbf{u}_{k}(\mathbf{r})}{\partial \mathbf{r}_{l}}+\frac{\partial \mathbf{u}_{l}(\mathbf{r})}{\partial \mathbf{r}_{k}}\right) \\
& =\frac{1}{2}\left(\sum_{k=1}^{3} \sum_{l=1}^{3} \mathbf{c}_{i j k l} \frac{\partial \mathbf{u}_{k}(\mathbf{r})}{\partial \mathbf{r}_{l}}+\sum_{k=1}^{3} \sum_{l=1}^{3} \mathbf{c}_{i j l k} \frac{\partial \mathbf{u}_{l}(\mathbf{r})}{\partial \mathbf{r}_{k}}\right)
\end{aligned}
$$

where the last equality uses the symmetric property of $\mathbf{c}$ :

$$
\mathbf{c}_{i j k l}=\mathbf{c}_{i j l k}
$$

Also note that

$$
\sum_{k=1}^{3} \sum_{l=1}^{3} \mathbf{c}_{i j k l} \frac{\partial \mathbf{u}_{k}(\mathbf{r})}{\partial \mathbf{r}_{l}}=\sum_{k=1}^{3} \sum_{l=1}^{3} \mathbf{c}_{i j l k} \frac{\partial \mathbf{u}_{l}(\mathbf{r})}{\partial \mathbf{r}_{k}}
$$

Thus, we have

$$
\boldsymbol{\sigma}_{i j}=\sum_{k=1}^{3} \sum_{l=1}^{3} \mathbf{c}_{i j k l} \frac{\partial \mathbf{u}_{k}(\mathbf{r})}{\partial \mathbf{r}_{l}}
$$

Remark Lemma 6.2 also implies that the stress tensor $\boldsymbol{\sigma}$ depends on position $\mathbf{r}$. In fact, strain tensor $\boldsymbol{\epsilon}$, stress tensor $\boldsymbol{\sigma}$ and elastic tensor $\mathbf{c}$ are spatial dependent while we suspend $\mathbf{r}$ in the notation, i.e.,

$$
\boldsymbol{\sigma}=\boldsymbol{\sigma}(\mathbf{r}), \quad \boldsymbol{\epsilon}=\boldsymbol{\epsilon}(\mathbf{r}) \quad \text { and } \quad \mathbf{c}=\mathbf{c}(\mathbf{r})
$$

The elastic tensor $\mathbf{c}$ is spacially independent when the elastic medium is homogeneous. From now on, however, we will always express tensors with their associated position $\mathbf{r}$ to avoid losing in the jungle of notations.

It has been proved that much of the Earth interior can be approximated by an isotropic solid type (Shearer 2009). If isotropy is assumed, the components of elastic tensor $\mathbf{c}(\mathbf{r})$ at position $\mathbf{r}$ can be written as

$$
\mathbf{c}_{i j k l}(\mathbf{r})=\lambda(\mathbf{r}) \delta_{i j} \delta_{k l}+\mu(\mathbf{r})\left(\delta_{i l} \delta_{j k}+\delta_{i k} \delta_{j l}\right),
$$


where $\mu(\mathbf{r})$ and $\lambda(\mathbf{r})$ are scalar physical quantities (in unit of $N / m^{2}$ ) describing the properties of medium at position $\mathbf{r}$ and are called Lamé coefficients; $\mu(\mathbf{r})$ measures the resistance of the material to shearing; $\lambda(\mathbf{r})$, however, does not have a simple physical implication; $\delta_{i j}, \delta_{k l}$, etc. are Kronecker delta, e.g.,

$$
\delta_{i j}= \begin{cases}0, & i \neq j \\ 1, & i=j\end{cases}
$$

The elastic tensor $\mathbf{c}(\mathbf{r})$ in isotropic case is in the form of the general 4-th rank isotropic tensor. We refer Theorem 7.4 in Matthews (2012) for the proof of equation (9). By plugging equation (9) into equation (6) and using the symmetry of strain tensor $\boldsymbol{\epsilon}(\mathbf{r})$, we obtain the constitutive equation for isotropic solid:

$$
\boldsymbol{\sigma}_{i j}(\mathbf{r})=\lambda(\mathbf{r}) \delta_{i j} \sum_{k=1}^{3} \boldsymbol{\epsilon}_{k k}(\mathbf{r})+2 \mu \boldsymbol{\epsilon}_{i j}(\mathbf{r}) .
$$

The matrix form of equation 11 is

$$
\boldsymbol{\sigma}(\mathbf{r})=\left[\begin{array}{ccc}
\lambda t r[\boldsymbol{\epsilon}(\mathbf{r})]+2 \mu \boldsymbol{\epsilon}_{11}(\mathbf{r}) & 2 \mu \boldsymbol{\epsilon}_{12}(\mathbf{r}) & 2 \mu \boldsymbol{\epsilon}_{13}(\mathbf{r}) \\
2 \mu \boldsymbol{\epsilon}_{21}(\mathbf{r}) & \lambda \operatorname{tr}[\boldsymbol{\epsilon}(\mathbf{r})]+2 \mu \boldsymbol{\epsilon}_{22}(\mathbf{r}) & 2 \mu \boldsymbol{\epsilon}_{23}(\mathbf{r}) \\
2 \mu \boldsymbol{\epsilon}_{31}(\mathbf{r}) & 2 \mu \boldsymbol{\epsilon}_{32}(\mathbf{r}) & \lambda \operatorname{tr}[\boldsymbol{\epsilon}(\mathbf{r})]+2 \mu \boldsymbol{\epsilon}_{33}(\mathbf{r})
\end{array}\right],
$$

where $\operatorname{tr}[\boldsymbol{\epsilon}(\mathbf{r})]=\sum_{k=1}^{3} \boldsymbol{\epsilon}_{k k}(\mathbf{r})$. Note that the stress tensor $\boldsymbol{\sigma}$ is symmetry due to the symmetry of strain tensor (i.e., $\left.\boldsymbol{\epsilon}_{i j}(\mathbf{r})=\boldsymbol{\epsilon}_{j i}(\mathbf{r})\right)$.

Equation (11) can be also written in terms of displacements by using equation (8):

$$
\boldsymbol{\sigma}_{i j}(\mathbf{r})=\lambda(\mathbf{r}) \delta_{i j} \sum_{k=1}^{3} \frac{\partial \mathbf{u}_{k}(\mathbf{r})}{\partial \mathbf{r}_{k}}+\mu(\mathbf{r})\left(\frac{\partial \mathbf{u}_{i}(\mathbf{r})}{\partial \mathbf{r}_{j}}+\frac{\partial \mathbf{u}_{j}(\mathbf{r})}{\partial \mathbf{r}_{i}}\right) .
$$

Remark The Lamé coefficients together with the density $\rho(\mathbf{r})$ give the compressional velocity $\left(v_{p}(\mathbf{r})\right)$ and shear velocity $\left(v_{s}(\mathbf{r})\right)$ at position $\mathbf{r}$ :

$$
v_{p}(\mathbf{r})=\sqrt{\frac{\lambda(\mathbf{r})+2 \mu(\mathbf{r})}{\rho(\mathbf{r})}}, \quad v_{s}(\mathbf{r})=\sqrt{\frac{\mu(\mathbf{r})}{\rho(\mathbf{r})}} .
$$

Note that the Lamé coefficients completely describe the linear relationship between stress and strain in an isotropic material and Lamé coefficients together with density give a complete description of isotropic elastic properties.

\section{Equation of Motion}

In this section we generalise the displacement function so that it is also time-dependent. We denote

$$
\mathbf{u}(\mathbf{r}, t)=\left(u_{x}(\mathbf{r}, t), u_{y}(\mathbf{r}, t), u_{z}(\mathbf{r}, t)\right)^{\top}=u_{x}(\mathbf{r}, t) \mathbf{e}^{1}+u_{y}(\mathbf{r}, t) \mathbf{e}^{2}+u_{z}(\mathbf{r}, t) \mathbf{e}^{3}
$$

to be the displacement of the point originating from position $\mathbf{r}$ at time $t$. Assume there is a continuous medium (or body) with volume $V \subset \mathbb{R}^{3}$, which is compact and has a boundary surface $S \subset V$, then the motion of each particle forming the body is determined by the forces acting in the interior of the body and the stresses acting on the surface of the body. According to the Newton's second law, the balance of linear momentum along the direction of unit vector $\mathbf{e}^{i}$ can be expressed by

$$
\int_{S} \mathbf{t}_{i}(\mathbf{r}, \mathbf{n}(\mathbf{r}), t) d S+\int_{V} \mathbf{f}_{i}(\mathbf{r}, t) d V=\int_{V} \mathbf{a}_{i}(\mathbf{r}, t) \rho(\mathbf{r}) d V, \quad i \in\{1,2,3\},
$$

where $\mathbf{t}_{i}(\mathbf{r}, \mathbf{n}(\mathbf{r}), t)$ is the $i$-th component of the stress acting on the surface element $d S$ (specified by its unit normal vector $\mathbf{n}(\mathbf{r}))$ at position $\mathbf{r}$ and time $t ; \mathbf{f}_{i}(\mathbf{r}, t)$ is the $i$-th component of the force per unit volume $\left(N / m^{3}\right)$ acting on the volume element $d V$ at position $\mathbf{r}$ and time $t ; \mathbf{a}_{i}(\mathbf{r}, t)$ is the $i$-th component of the acceleration of particle at position $\mathbf{r}$ 
and time $t ; \rho(\mathbf{r})$ is the density of the body and is constant with time. The Gauchy's Stress Theorem (4) allows us to rewrite the equation 13 as

$$
\int_{S} \sum_{j=1}^{3} \boldsymbol{\sigma}_{i j}(\mathbf{r}, t) \mathbf{n}_{j}(\mathbf{r}) d S+\int_{V} \mathbf{f}_{i}(\mathbf{r}, t) d V=\int_{V} \mathbf{a}_{i}(\mathbf{r}, t) \rho(\mathbf{r}) d V,
$$

where $\boldsymbol{\sigma}_{i j}(\mathbf{r}, t)$ is the coordinate stress located on the $i$-th row and $j$-th column of the stress tensor $\boldsymbol{\sigma}(\mathbf{r}, t)$ at position $\mathbf{r}$ and time $t$. Note that the particle acceleration $\mathbf{a}_{i}(\mathbf{r}, t)$ can be expressed as

$$
\mathbf{a}_{i}(\mathbf{r}, t)=\frac{\partial^{2} \mathbf{u}_{i}(\mathbf{r}, t)}{\partial t^{2}}=\ddot{\mathbf{u}}_{i}(\mathbf{r}, t) .
$$

Thus, equation 14 becomes

$$
\int_{S} \sum_{j=1}^{3} \boldsymbol{\sigma}_{i j}(\mathbf{r}, t) \mathbf{n}_{j}(\mathbf{r}) d S+\int_{V} \mathbf{f}_{i}(\mathbf{r}, t) d V=\int_{V} \ddot{\mathbf{u}}_{i}(\mathbf{r}, t) \rho(\mathbf{r}) d V .
$$

The equation (15) is the equation of motion for a finite medium. Due to the Divergence Theorem (or Gauss's Theorem), the surface integral in equation 15 can be replaced by a volume integral:

$$
\int_{V} \sum_{j=1}^{3} \frac{\partial \boldsymbol{\sigma}_{i j}(\mathbf{r}, t)}{\partial \mathbf{r}_{j}} d V+\int_{V} \mathbf{f}_{i}(\mathbf{r}, t) d V=\int_{V} \ddot{\mathbf{u}}_{i}(\mathbf{r}, t) \rho(\mathbf{r}) d V .
$$

Thus, the equation of motion for an infinite medium can be written as the differential equation by cancelling the integrals on both sides of equation (16):

$$
\sum_{j=1}^{3} \frac{\partial \boldsymbol{\sigma}_{i j}(\mathbf{r}, t)}{\partial \mathbf{r}_{j}}+\mathbf{f}_{i}(\mathbf{r}, t)=\ddot{\mathbf{u}}_{i}(\mathbf{r}, t) \rho(\mathbf{r}) .
$$

For an elastic medium, Lemma 6.2 allows us to express the equation (17) in terms of displacements:

$$
\sum_{j=1}^{3} \frac{\partial}{\partial \mathbf{r}_{j}}\left(\sum_{k=1}^{3} \sum_{l=1}^{3} \mathbf{c}_{i j k l}(\mathbf{r}) \frac{\partial \mathbf{u}_{k}(\mathbf{r}, t)}{\partial \mathbf{r}_{l}}\right)+\mathbf{f}_{i}(\mathbf{r}, t)=\ddot{\mathbf{u}}_{i}(\mathbf{r}, t) \rho(\mathbf{r}) .
$$

For an isotropic elastic medium, using equation (12) the differential equation of motion (17) can be written as

$$
\sum_{j=1}^{3} \frac{\partial}{\partial \mathbf{r}_{j}}\left(\lambda(\mathbf{r}) \delta_{i j} \sum_{k=1}^{3} \frac{\partial \mathbf{u}_{k}(\mathbf{r}), t}{\partial \mathbf{r}_{k}}+\mu(\mathbf{r})\left(\frac{\partial \mathbf{u}_{i}(\mathbf{r})}{\partial \mathbf{r}_{j}}+\frac{\partial \mathbf{u}_{j}(\mathbf{r})}{\partial \mathbf{r}_{i}}\right)\right)+\mathbf{f}_{i}(\mathbf{r}, t)=\ddot{\mathbf{u}}_{i}(\mathbf{r}, t) \rho(\mathbf{r}) .
$$

Note that under the assumption of homogeneity (i.e., $\lambda(\mathbf{r})=\lambda, \mu(\mathbf{r})=\mu$ and $\rho(\mathbf{r})=\rho$ are constant in space) the first term of equation (19) can be rewritten as follow:

$$
\begin{aligned}
& \sum_{j=1}^{3} \frac{\partial}{\partial \mathbf{r}_{j}}\left(\lambda \delta_{i j} \sum_{k=1}^{3} \frac{\partial \mathbf{u}_{k}(\mathbf{r}, t)}{\partial \mathbf{r}_{k}}+\mu\left(\frac{\partial \mathbf{u}_{i}(\mathbf{r}, t)}{\partial \mathbf{r}_{j}}+\frac{\partial \mathbf{u}_{j}(\mathbf{r}, t)}{\partial \mathbf{r}_{i}}\right)\right) \\
= & \lambda \sum_{j=1}^{3} \delta_{i j} \sum_{k=1}^{3} \frac{\partial^{2} \mathbf{u}_{k}(\mathbf{r}, t)}{\partial \mathbf{r}_{j} \partial \mathbf{r}_{k}}+\mu \sum_{j=1}^{3} \frac{\partial^{2} \mathbf{u}_{i}(\mathbf{r}, t)}{\partial \mathbf{r}_{j}^{2}}+\mu \sum_{j=1}^{3} \frac{\partial^{2} \mathbf{u}_{j}(\mathbf{r}, t)}{\partial \mathbf{r}_{i} \partial \mathbf{r}_{j}} \\
= & \lambda \sum_{k=1}^{3} \frac{\partial^{2} \mathbf{u}_{k}(\mathbf{r}, t)}{\partial \mathbf{r}_{i} \partial \mathbf{r}_{k}}+\mu \sum_{j=1}^{3} \frac{\partial^{2} \mathbf{u}_{i}(\mathbf{r}, t)}{\partial \mathbf{r}_{j}^{2}}+\mu \sum_{j=1}^{3} \frac{\partial^{2} \mathbf{u}_{j}(\mathbf{r}, t)}{\partial \mathbf{r}_{i} \partial \mathbf{r}_{j}} \\
= & (\lambda+\mu) \sum_{k=1}^{3} \frac{\partial^{2} \mathbf{u}_{k}(\mathbf{r}, t)}{\partial \mathbf{r}_{i} \partial \mathbf{r}_{k}}+\mu \sum_{j=1}^{3} \frac{\partial^{2} \mathbf{u}_{i}(\mathbf{r}, t)}{\partial \mathbf{r}_{j}^{2}}
\end{aligned}
$$

We thus obtain

$$
(\lambda+\mu) \sum_{k=1}^{3} \frac{\partial^{2} \mathbf{u}_{k}(\mathbf{r}, t)}{\partial \mathbf{r}_{i} \partial \mathbf{r}_{k}}+\mu \sum_{j=1}^{3} \frac{\partial^{2} \mathbf{u}_{i}(\mathbf{r}, t)}{\partial \mathbf{r}_{j}^{2}}+\mathbf{f}_{i}(\mathbf{r}, t)=\ddot{\mathbf{u}}_{i}(\mathbf{r}, t) \rho
$$


The differential equation of motion 20 for an isotropic homogeneous elastic medium can also be expressed in a more compact form using vector notation:

$$
(\lambda+\mu) \nabla(\nabla \cdot \mathbf{u}(\mathbf{r}, t))+\mu \nabla^{2} \mathbf{u}(\mathbf{r}, t)+\mathbf{f}(\mathbf{r}, t)=\rho \ddot{\mathbf{u}}(\mathbf{r}, t),
$$

where

- $\ddot{\mathbf{u}}(\mathbf{r}, t)=\left(\ddot{\mathbf{u}}_{1}(\mathbf{r}, t), \ddot{\mathbf{u}}_{2}(\mathbf{r}, t), \ddot{\mathbf{u}}_{3}(\mathbf{r}, t)\right)^{\top}=\left(\frac{\partial^{2} \mathbf{u}_{1}(\mathbf{r}, t)}{\partial t^{2}}, \frac{\partial^{2} \mathbf{u}_{2}(\mathbf{r}, t)}{\partial t^{2}}, \frac{\partial^{2} \mathbf{u}_{3}(\mathbf{r}, t)}{\partial t^{2}}\right)^{\top} ;$

- $\nabla \cdot \mathbf{u}(\mathbf{r}, t)=\sum_{k=1}^{3} \frac{\partial \mathbf{u}_{k}(\mathbf{r}, t)}{\partial \mathbf{r}_{k}}$ is the divergence of the displacement vector $\mathbf{u}(\mathbf{r}, t)$;

- $\nabla(\nabla \cdot \mathbf{u}(\mathbf{r}, t))=\left(\sum_{k=1}^{3} \frac{\partial^{2} \mathbf{u}_{k}(\mathbf{r}, t)}{\partial \mathbf{r}_{1} \partial \mathbf{r}_{k}}, \sum_{k=1}^{3} \frac{\partial^{2} \mathbf{u}_{k}(\mathbf{r}, t)}{\partial \mathbf{r}_{2} \partial \mathbf{r}_{k}}, \sum_{k=1}^{3} \frac{\partial^{2} \mathbf{u}_{k}(\mathbf{r}, t)}{\partial \mathbf{r}_{3} \partial \mathbf{r}_{k}}\right)^{\top}$ is the gradient of the scalar $\nabla$ $\mathbf{u}(\mathbf{r}, t)$;

- $\nabla^{2} \mathbf{u}(\mathbf{r}, t)=\left(\nabla^{2} \mathbf{u}_{1}(\mathbf{r}, t), \nabla^{2} \mathbf{u}_{2}(\mathbf{r}, t), \nabla^{2} \mathbf{u}_{3}(\mathbf{r}, t)\right)^{\top}$ is the Laplacian of the displacement vector $\mathbf{u}(\mathbf{r}, t)$

- $\nabla^{2} \mathbf{u}_{i}(\mathbf{r}, t)=\nabla \cdot \nabla \mathbf{u}_{i}(\mathbf{r}, t)$ for all $i \in\{1,2,3\}$ is the Laplacian of the scalar $\mathbf{u}_{i}(\mathbf{r}, t)$.

\section{Green's Tensors}

Assume that the system (or field) of volume forces

$$
\mathbf{f}=\{\mathbf{f}(\mathbf{r}, t): \mathbf{r} \in V, t \in \mathbb{R}\}
$$

in a medium with volume $V$ and surface $S$ is formed by an impulsive force acting at position $\mathbf{r}=\boldsymbol{\xi}$ and time $t=\tau$ along the direction of unit vector $\mathbf{e}^{n}$ with some $n \in\{1,2,3\}$, i.e.,

$$
\mathbf{f}_{i}(\mathbf{r}, t)=A_{n} \delta(\mathbf{r}-\boldsymbol{\xi}) \delta(t-\tau) \delta_{i n} \quad \forall i \in\{1,2,3\},
$$

where $A_{n}$ (in unit of $N s$ ) is a measurement of the magnitude of the impulsive force $\mathbf{f}_{n}(\boldsymbol{\xi}, \tau) ; \delta(\mathbf{r}-\boldsymbol{\xi})$ with unit $m^{-3}$ and $\delta(t-\tau)$ with unit $s^{-1}$ are Dirac delta functions indicating that there is a volume force (impulsive force) applied at position $\mathbf{r}=\boldsymbol{\xi}$ and time $t=\tau$ and volume forces outside this position and time are zero; $\delta_{\text {in }}$ is the Kronecker delta (see equation (10) ) indicating that the direction of the impulsive force is along that of $\mathbf{e}^{n}$ for some $n \in\{1,2,3\}$. By substituting equation (22) into the equation of motion 15 for a finite medium, we have

$$
\int_{S} \sum_{j=1}^{3} \boldsymbol{\sigma}_{i j}(\mathbf{r}, t) \mathbf{n}_{j}(\mathbf{r}) d S+\int_{V} A_{n} \delta(\mathbf{r}-\boldsymbol{\xi}) \delta(t-\tau) \delta_{i n} d V=\int_{V} \frac{\partial^{2} \mathbf{u}_{i}(\mathbf{r}, t)}{\partial t^{2}} \rho(\mathbf{r}) d V .
$$

For an elastic medium, we then can express $\boldsymbol{\sigma}_{i j}(\mathbf{r}, t)$ in equation [23) in terms of displacements:

$$
\int_{S} \sum_{j=1}^{3} \mathbf{n}_{j}(\mathbf{r}) \sum_{k=1}^{3} \sum_{l=1}^{3} \mathbf{c}_{i j k l}(\mathbf{r}) \frac{\partial \mathbf{u}_{k}(\mathbf{r}, t)}{\partial \mathbf{r}_{l}} d S+\int_{V} A_{n} \delta(\mathbf{r}-\boldsymbol{\xi}) \delta(t-\tau) \delta_{i n} d V=\int_{V} \frac{\partial^{2} \mathbf{u}_{i}(\mathbf{r}, t)}{\partial t^{2}} \rho(\mathbf{r}) d V .
$$

We divide both side of equation (24) by $A_{n}$ and obtain

$$
\int_{S} \sum_{j=1}^{3} \mathbf{n}_{j}(\mathbf{r}) \sum_{k=1}^{3} \sum_{l=1}^{3} \mathbf{c}_{i j k l}(\mathbf{r}) \frac{\partial \frac{\underline{\mathbf{u}}_{k}(\mathbf{r}, t)}{A_{n}}}{\partial \mathbf{r}_{l}} d S+\int_{V} \delta(\mathbf{r}-\boldsymbol{\xi}) \delta(t-\tau) \delta_{i n} d V=\int_{V} \frac{\partial^{2} \frac{\mathbf{u}_{i}(\mathbf{r}, t)}{A_{n}}}{\partial t^{2}} \rho(\mathbf{r}) d V .
$$

Now define a second-order (or -rank) tensor $\mathbf{G}(\mathbf{r}, t ; \boldsymbol{\xi}, \tau) \in \mathbb{R}^{3 \times 3}$ with

$$
\mathbf{G}_{i j}(\mathbf{r}, t ; \boldsymbol{\xi}, \tau)=\frac{\mathbf{u}_{i}(\mathbf{r}, t)}{A_{j}}, \quad i, j \in\{1,2,3\}
$$

which reads as the value of $i$-th component of the displacement (i.e., the displacement along the direction of $\mathbf{e}^{i}$ ) at position $\mathbf{r}$ and time $t$ due to the unit impulsive force applied at position $\boldsymbol{\xi}$ and time $\tau$ along the direction of $\mathbf{e}^{j}$. Thus $\mathbf{G}(\mathbf{r}, t ; \boldsymbol{\xi}, \tau)$ gives the relationship between the elastic displacement and an unit impulsive force in space and time and 
we call $\mathbf{G}(\mathbf{r}, t ; \boldsymbol{\xi}, \tau)$ the Green's tensor of elastodynamics (Udías et al.2014). Putting the Green's tensor 26) into equation (25), we have the equation of motion for a finite elastic medium in terms of Green's tensor:

$$
\int_{S} \sum_{j=1}^{3} \mathbf{n}_{j}(\mathbf{r}) \sum_{k=1}^{3} \sum_{l=1}^{3} \mathbf{c}_{i j k l}(\mathbf{r}) \frac{\partial \mathbf{G}_{k n}(\mathbf{r}, t ; \boldsymbol{\xi}, \tau)}{\partial \mathbf{r}_{l}} d S+\int_{V} \delta(\mathbf{r}-\boldsymbol{\xi}) \delta(t-\tau) \delta_{i n} d V=\int_{V} \frac{\partial^{2} \mathbf{G}_{i n}(\mathbf{r}, t ; \boldsymbol{\xi}, \tau)}{\partial t^{2}} \rho(\mathbf{r}) d V .
$$

Using the Divergence theorem, we can also obtain the equation of motion for an infinite elastic medium in terms of Green's tensor:

$$
\sum_{j=1}^{3} \frac{\partial}{\partial \mathbf{r}_{j}}\left(\sum_{k=1}^{3} \sum_{l=1}^{3} \mathbf{c}_{i j k l}(\mathbf{r}) \frac{\partial \mathbf{G}_{k n}(\mathbf{r}, t ; \boldsymbol{\xi}, \tau)}{\partial \mathbf{r}_{l}}\right)+\delta(\mathbf{r}-\boldsymbol{\xi}) \delta(t-\tau) \delta_{i n}=\frac{\partial^{2} \mathbf{G}_{i n}(\mathbf{r}, t ; \boldsymbol{\xi}, \tau)}{\partial t^{2}} \rho(\mathbf{r}) .
$$

If we assume that there is no time dependence in equation of motion (15) for a finite medium, that is

$$
\begin{aligned}
\int_{S} \sum_{j=1}^{3} \boldsymbol{\sigma}_{i j}(\mathbf{r}) \mathbf{n}_{j}(\mathbf{r}) d S+\int_{V} \mathbf{f}_{i}(\mathbf{r}) d V & =\int_{V} \frac{\partial^{2} \mathbf{u}_{i}(\mathbf{r})}{\partial t^{2}} \rho(\mathbf{r}) d V \\
& =0
\end{aligned}
$$

with

$$
\mathbf{f}_{i}(\mathbf{r})=A_{n} \delta(\mathbf{r}-\boldsymbol{\xi}) \delta_{i n} \quad \forall i \in\{1,2,3\}
$$

the system of volume forces in a medium formed by an impulsive force acting at position $\mathbf{r}=\boldsymbol{\xi}$ along the direction of the unit vector $\mathbf{e}^{n}$ with some $n \in\{1,2,3\}$, then equation 27) and 28) give that

$$
\int_{S} \sum_{j=1}^{3} \boldsymbol{\sigma}_{i j}(\mathbf{r}) \mathbf{n}_{j}(\mathbf{r}) d S+\int_{V} A_{n} \delta(\mathbf{r}-\boldsymbol{\xi}) \delta_{i n} d V=0
$$

In an elastic medium, we then have

$$
\int_{S} \sum_{j=1}^{3} \mathbf{n}_{j}(\mathbf{r}) \sum_{k=1}^{3} \sum_{l=1}^{3} \mathbf{c}_{i j k l}(\mathbf{r}) \frac{\partial \mathbf{u}_{k}(\mathbf{r})}{\partial \mathbf{r}_{l}} d S+\int_{V} A_{n} \delta(\mathbf{r}-\boldsymbol{\xi}) \delta_{i n} d V=0 .
$$

Define a second-order tensor $\mathbf{S}(\mathbf{r} ; \boldsymbol{\xi}) \in \mathbb{R}^{3 \times 3}$ with

$$
\mathbf{S}_{i j}(\mathbf{r} ; \boldsymbol{\xi})=\frac{\mathbf{u}_{i}(\mathbf{r})}{A_{j}}, \quad i, j \in\{1,2,3\},
$$

the value of $i$-th component of the displacement at position $\mathbf{r}$ due to the unit impulsive force applied at position $\boldsymbol{\xi}$ along the direction of $\mathbf{e}^{j}$. Thus, the tensor $\mathbf{S}(\mathbf{r} ; \boldsymbol{\xi})$ represents the elastic displacement produced by an unit impulsive force in static case and we call it the Green's tensor of elastostatics. Finally, we obtain the equation of motion for a finite elastic medium:

$$
\int_{S} \sum_{j=1}^{3} \mathbf{n}_{j}(\mathbf{r}) \sum_{k=1}^{3} \sum_{l=1}^{3} \mathbf{c}_{i j k l}(\mathbf{r}) \frac{\partial \mathbf{S}_{k n}(\mathbf{r} ; \boldsymbol{\xi})}{\partial \mathbf{r}_{l}} d S+\int_{V} \delta(\mathbf{r}-\boldsymbol{\xi}) \delta_{i n} d V=0
$$

and equation of motion for an infinite elastic medium:

$$
\sum_{j=1}^{3} \frac{\partial}{\partial \mathbf{r}_{j}}\left(\sum_{k=1}^{3} \sum_{l=1}^{3} \mathbf{c}_{i j k l}(\mathbf{r}) \frac{\partial \mathbf{S}_{k n}(\mathbf{r} ; \boldsymbol{\xi})}{\partial \mathbf{r}_{l}}\right)+\delta(\mathbf{r}-\boldsymbol{\xi}) \delta_{i n}=0
$$

in static case.

\section{Initial and Boundary Conditions}

In elastodynamic case, the displacements $\mathbf{u}(\mathbf{r}, t)$ throughout a medium with volume $V$ and surface $S$ caused by a system of volume forces $\mathbf{f}$ during a time interval $\left[T_{0}, T_{1}\right]$ are found by given 


\section{- Initial conditions:}

$$
\begin{aligned}
& \mathbf{u}\left(\mathbf{r}, T_{0}\right)=\hat{\mathbf{u}}(\mathbf{r}), \\
& \dot{\mathbf{u}}\left(\mathbf{r}, T_{0}\right)=\hat{\dot{\mathbf{u}}}(\mathbf{r})
\end{aligned}
$$

for all $\mathbf{r} \in V$, where $\hat{\mathbf{u}}(\mathbf{r})$ and $\hat{\dot{\mathbf{u}}}(\mathbf{r})$ are respectively the given values of displacements and velocities at initial time $t=T_{0}$.

- Boundary conditions: The boundary conditions can be divided into three types:

1. Displacement (or Dirichlet) boundary condition:

$$
\mathbf{u}(\mathbf{r}, t)=\tilde{\mathbf{u}}(\mathbf{r}, t)
$$

for all $\mathbf{r} \in S$ and $t \in\left[T_{0}, T_{1}\right]$, where $\tilde{\mathbf{u}}(\mathbf{r}, t)$ is the given values of displacements on the body surface element $d S$ during $\left[T_{0}, T_{1}\right]$;

2. Traction (or Neumann) boundary condition:

$$
\mathbf{t}(\mathbf{r}, \mathbf{n}(\mathbf{r}), t)=\tilde{\mathbf{t}}(\mathbf{r}, \mathbf{n}(\mathbf{r}), t)
$$

for all $\mathbf{r} \in S$ and $t \in\left[T_{0}, T_{1}\right]$, where $\tilde{\mathbf{t}}(\mathbf{r}, \mathbf{n}(\mathbf{r}), t)$ is the given values of stresses on the body surface element $d S$ during $\left[T_{0}, T_{1}\right]$;

3. Mixed boundary condition:

$$
\mathbf{u}(\mathbf{r}, t)=\tilde{\mathbf{u}}(\mathbf{r}, t)
$$

for all $\mathbf{r} \in S_{1}$ and $t \in\left[T_{0}, T_{1}\right]$ and

$$
\mathbf{t}(\mathbf{r}, \mathbf{n}(\mathbf{r}), t)=\tilde{\mathbf{t}}(\mathbf{r}, \mathbf{n}(\mathbf{r}), t)
$$

for all $\mathbf{r} \in S_{2}$ and $t \in\left[T_{0}, T_{1}\right]$, where $S_{1}$ and $S_{2}$ are two disjoint subsets of $S$ :

$$
S_{1} \cup S_{2}=S \quad \text { and } \quad S_{1} \cap S_{2}=\emptyset
$$

Similarly, we can set three types of boundary conditions for elastostatic case where time element is neglected for displacements $\mathbf{u}(\mathbf{r})$ throughout a body with volume $V$ and surface $S$ caused by a system of time independent volume forces $\mathbf{f}(\mathbf{r})$ :

1. Displacement boundary condition:

$$
\mathbf{u}(\mathbf{r})=\tilde{\mathbf{u}}(\mathbf{r})
$$

for all $\mathbf{r} \in S$;

2. Traction boundary condition:

$$
\mathbf{t}(\mathbf{r}, \mathbf{n}(\mathbf{r}))=\tilde{\mathbf{t}}(\mathbf{r}, \mathbf{n}(\mathbf{r}))
$$

for all $\mathbf{r} \in S$;

3. Mixed boundary condition:

$$
\mathbf{u}(\mathbf{r})=\tilde{\mathbf{u}}(\mathbf{r})
$$

for all $\mathbf{r} \in S_{1}$ and

$$
\mathbf{t}(\mathbf{r}, \mathbf{n}(\mathbf{r}))=\tilde{\mathbf{t}}(\mathbf{r}, \mathbf{n}(\mathbf{r}))
$$

for all $\mathbf{r} \in S_{2}$, where $S_{1}$ and $S_{2}$ are two disjoint subsets of $S$ :

$$
S_{1} \cup S_{2}=S \quad \text { and } \quad S_{1} \cap S_{2}=\emptyset .
$$

Remark Note that if $S_{1}=\emptyset$, the mixed boundary condition becomes the traction boundary condition; if $S_{2}=\emptyset$, the mixed boundary condition becomes the displacement boundary condition. 


\section{Principle of Superposition}

Before we present the superposition principle, we recall and define some important concepts that will be used frequently in the rest of the notes. Assume that there is a continuous elastic medium with volume $V$ and surface $S$. Then we define following concepts:

- Density field, denoted by

$$
\varrho=\{\rho(\mathbf{r}): \mathbf{r} \in V\},
$$

is a set of densities $\rho(\mathbf{r})$ at all positions $\mathbf{r} \in V$;

- Elastic tensor field, denoted by

$$
\mathbf{c}=\{\mathbf{c}(\mathbf{r}): \mathbf{r} \in V\},
$$

is a set of elastic tensors $\mathbf{c}(\mathbf{r})$ at all positions $\mathbf{r} \in V$;

- Strain tensor field, denoted by

$$
\boldsymbol{\epsilon}=\left\{\boldsymbol{\epsilon}(\mathbf{r}, t): \mathbf{r} \in V, t \in\left[T_{0}, T_{1}\right]\right\},
$$

is a set of strain tensors $\boldsymbol{\epsilon}(\mathbf{r}, t)$ at all positions $\mathbf{r} \in V$ during the time interval $\left[T_{0}, T_{1}\right]$;

- Field of volume forces, denoted by

$$
\mathbf{f}=\left\{\mathbf{f}(\mathbf{r}, t): \mathbf{r} \in V, t \in\left[T_{0}, T_{1}\right]\right\},
$$

is a set of volume forces $\mathbf{f}(\mathbf{r}, t)$ at all positions $\mathbf{r} \in V$ during the time interval $\left[T_{0}, T_{1}\right]$;

- Displacement field, denoted by

$$
\mathbf{u}=\left\{\mathbf{u}(\mathbf{r}, t): \mathbf{r} \in V, t \in\left[T_{0}, T_{1}\right]\right\},
$$

is a set of displacements $\mathbf{u}(\mathbf{r}, t)$ at all positions $\mathbf{r} \in V$ during the time interval $\left[T_{0}, T_{1}\right]$;

- Displacement field set, denoted by

$$
\mathcal{E}\left(\varrho, \mathbf{c}, \mathbf{f}, S \times V \times\left[T_{0}, T_{1}\right]\right)
$$

is a set of all displacement fields that satisfy the equation of motion $(18)$ with density field $\varrho$, elastic tensor field $\mathbf{c}$ and volume force field $\mathbf{f}$ on the medium with volume $V$ and surface $S$ during the time interval $\left[T_{0}, T_{1}\right]$.

Proposition 10.1 (Principle of Superposition) Assume that there is a continuous elastic medium (with volume $V$ and surface $S=S_{1} \cup S_{2}$ ) specified by the density field @ and the elastic tensor field $\mathbf{c}$. Let $\mathbf{f}$ and $\mathbf{g}$ be two fields of volume forces acting to the medium during the time interval $\left[T_{0}, T_{1}\right]$, while let

$$
\begin{gathered}
\mathbf{u} \in \mathcal{E}\left(\varrho, \mathbf{c}, \mathbf{f}, V \times S \times\left[T_{0}, T_{1}\right]\right), \\
\mathbf{w} \in \mathcal{E}\left(\varrho, \mathbf{c}, \mathbf{g}, V \times S \times\left[T_{0}, T_{1}\right]\right)
\end{gathered}
$$

be the displacement fields caused respectively by $\mathbf{f}$ and $\mathbf{g}$ under the initial conditions:

$$
\begin{array}{lll}
\mathbf{u}\left(\mathbf{r}, T_{0}\right)=\hat{\mathbf{u}}(\mathbf{r}), & \mathbf{w}\left(\mathbf{r}, T_{0}\right)=\hat{\mathbf{w}}(\mathbf{r}) & \forall \mathbf{r} \in V \\
\dot{\mathbf{u}}\left(\mathbf{r}, T_{0}\right)=\hat{\dot{\mathbf{u}}}(\mathbf{r}), & \dot{\mathbf{w}}\left(\mathbf{r}, T_{0}\right)=\hat{\dot{\mathbf{w}}}(\mathbf{r}) & \forall \mathbf{r} \in V
\end{array}
$$

and boundary conditions

$$
\begin{aligned}
\mathbf{u}(\mathbf{r}, t) & =\tilde{\mathbf{u}}(\mathbf{r}, t) & & \forall \mathbf{r} \in S_{1} & \text { and } & \forall t \in\left[T_{0}, T_{1}\right] \\
\mathbf{w}(\mathbf{r}, t) & =\tilde{\mathbf{w}}(\mathbf{r}, t) & & \forall \mathbf{r} \in S_{1} & \text { and } & \forall t \in\left[T_{0}, T_{1}\right] \\
\mathbf{t}(\mathbf{r}, \mathbf{n}(\mathbf{r}), t, \mathbf{u}) & =\tilde{\mathbf{t}}(\mathbf{r}, \mathbf{n}(\mathbf{r}), t, \mathbf{u}) & & \forall \mathbf{r} \in S_{2} & \text { and } & \forall t \in\left[T_{0}, T_{1}\right] \\
\mathbf{t}(\mathbf{r}, \mathbf{n}(\mathbf{r}), t, \mathbf{w}) & =\tilde{\mathbf{t}}(\mathbf{r}, \mathbf{n}(\mathbf{r}), t, \mathbf{w}) & & \forall \mathbf{r} \in S_{2} & \text { and } & \forall t \in\left[T_{0}, T_{1}\right],
\end{aligned}
$$

where $\mathbf{t}(\mathbf{r}, \mathbf{n}(\mathbf{r}), t, \mathbf{u})$ and $\mathbf{t}(\mathbf{r}, \mathbf{n}(\mathbf{r}), t, \mathbf{w})$ are stresses (due to displacement fields $\mathbf{u}$ and $\mathbf{w}$ respectively) acting on the surface element $d S$ at position $\mathbf{r}$ and time $t$. 
The superposition principle then shows that for constants $\alpha$ and $\beta$ the displacement field

$$
\mathbf{v}=(\alpha \mathbf{u}+\beta \mathbf{w}) \in \mathcal{E}\left(\varrho, \mathbf{c}, \alpha \mathbf{f}+\beta \mathbf{g}, V \times S \times\left[T_{0}, T_{1}\right]\right)
$$

under the initial condition

$$
\mathbf{v}\left(\mathbf{r}, T_{0}\right)=\alpha \hat{\mathbf{u}}(\mathbf{r})+\beta \hat{\mathbf{w}}(\mathbf{r}), \quad \dot{\mathbf{v}}\left(\mathbf{r}, T_{0}\right)=\alpha \hat{\dot{\mathbf{u}}}(\mathbf{r})+\beta \hat{\dot{\mathbf{w}}}(\mathbf{r}) \quad \forall \mathbf{r} \in V
$$

and boundary conditions

$$
\begin{aligned}
& \mathbf{v}(\mathbf{r}, t)=\alpha \tilde{\mathbf{u}}(\mathbf{r}, t)+\beta \tilde{\mathbf{w}}(\mathbf{r}, t) \quad \forall \mathbf{r} \in S_{1} \quad \text { and } \quad \forall t \in\left[T_{0}, T_{1}\right] \\
& \mathbf{t}(\mathbf{r}, \mathbf{n}(\mathbf{r}), t, \mathbf{v})=\alpha \tilde{\mathbf{t}}(\mathbf{r}, \mathbf{n}(\mathbf{r}), t, \mathbf{u})+\beta \tilde{\mathbf{t}}(\mathbf{r}, \mathbf{n}(\mathbf{r}), t, \mathbf{w}) \quad \forall \mathbf{r} \in S_{2} \quad \text { and } \quad \forall t \in\left[T_{0}, T_{1}\right] .
\end{aligned}
$$

Proof The proof is an immediate result from the linearity of the equation of motion [18,.

The linearity property stated by the principle of superposition is an important result, which will be used to prove the uniqueness theorems in the next section.

\section{Uniqueness Theorems}

In this section we show the uniqueness of displacement fields given different boundary conditions in both elstodynamic and elastostatic cases.

Before presenting proofs, we define the positive-definiteness of elastic tensor field c and the rate of mechanical work:

Definition 11.1 (Positive-Definiteness of Elastic Tensor Field) The elastic tensor field $\mathbf{c}$ is positive-definite if it satisfies

$$
\sum_{i=1}^{3} \sum_{j=1}^{3} \sum_{k=1}^{3} \sum_{l=1}^{3} \mathbf{c}_{i j k l}(\mathbf{r}) \boldsymbol{\zeta}_{i j}(\mathbf{r}) \boldsymbol{\zeta}_{k l}(\mathbf{r})>0
$$

for arbitrary non-zero symmetric tensors $\zeta(\mathbf{r})$ at all positions $\mathbf{r} \in V$.

Definition 11.2 (Rate of Mechanical Work) The rate of of mechanical work $R_{W}(\mathbf{r}, t)$ at position $\mathbf{r}$ and time $t$ is defined as

$$
R_{W}(\mathbf{r}, t)=\int_{V} \mathbf{f}(\mathbf{r}, t) \cdot \dot{\mathbf{u}}(\mathbf{r}, t) d V+\int_{S} \mathbf{t}(\mathbf{r}, \mathbf{n}(\mathbf{r}), t, \mathbf{u}) \cdot \dot{\mathbf{u}}(\mathbf{r}, t) d S,
$$

where

- $\dot{\mathbf{u}}(\mathbf{r}, t)=\left(\frac{\partial \mathbf{u}_{1}(\mathbf{r}, t)}{\partial t}, \frac{\partial \mathbf{u}_{2}(\mathbf{r}, t)}{\partial t}, \frac{\partial \mathbf{u}_{3}(\mathbf{r}, t)}{\partial t}\right)^{\top}$

- $\mathbf{f}(\mathbf{r}, t) \cdot \dot{\mathbf{u}}(\mathbf{r}, t)$ is the dot product of $\mathbf{f}(\mathbf{r}, t)$ and $\dot{\mathbf{u}}(\mathbf{r}, t)$;

- $\mathbf{t}(\mathbf{r}, \mathbf{n}(\mathbf{r}), t, \mathbf{u}) \cdot \dot{\mathbf{u}}(\mathbf{r}, t)$ is the dot product of $\mathbf{t}(\mathbf{r}, \mathbf{n}(\mathbf{r}), t, \mathbf{u})$ and $\dot{\mathbf{u}}(\mathbf{r}, t)$. 
Note that the equation (29) for the rate of mechanical work can be further transformed as follow:

$$
\begin{aligned}
& R_{W}(\mathbf{r}, t)=\int_{V} \mathbf{f}(\mathbf{r}, t) \cdot \dot{\mathbf{u}}(\mathbf{r}, t) d V+\int_{S} \mathbf{t}(\mathbf{r}, \mathbf{n}(\mathbf{r}), t, \mathbf{u}) \cdot \dot{\mathbf{u}}(\mathbf{r}, t) d S \\
& =\int_{V} \sum_{i=1}^{3} \mathbf{f}_{i}(\mathbf{r}, t) \dot{\mathbf{u}}_{i}(\mathbf{r}, t) d V+\int_{S} \sum_{i=1}^{3} \mathbf{t}_{i}(\mathbf{r}, \mathbf{n}(\mathbf{r}), t, \mathbf{u}) \dot{\mathbf{u}}_{i}(\mathbf{r}, t) d S \\
& =\int_{V} \sum_{i=1}^{3} \mathbf{f}_{i}(\mathbf{r}, t) \dot{\mathbf{u}}_{i}(\mathbf{r}, t) d V+\int_{S} \sum_{i=1}^{3} \sum_{j=1}^{3} \boldsymbol{\sigma}_{i j}(\mathbf{r}, t) \mathbf{n}_{j}(\mathbf{r}) \dot{\mathbf{u}}_{i}(\mathbf{r}, t) d S \\
& =\int_{V} \sum_{i=1}^{3} \mathbf{f}_{i}(\mathbf{r}, t) \dot{\mathbf{u}}_{i}(\mathbf{r}, t) d V+\int_{V} \sum_{i=1}^{3} \sum_{j=1}^{3} \frac{\partial}{\partial \mathbf{r}_{j}}\left(\boldsymbol{\sigma}_{i j}(\mathbf{r}, t) \dot{\mathbf{u}}_{i}(\mathbf{r}, t)\right) d V \\
& =\int_{V}\left(\sum_{i=1}^{3} \mathbf{f}_{i}(\mathbf{r}, t) \dot{\mathbf{u}}_{i}(\mathbf{r}, t)+\sum_{i=1}^{3} \sum_{j=1}^{3} \frac{\partial \boldsymbol{\sigma}_{i j}(\mathbf{r}, t)}{\partial \mathbf{r}_{j}} \dot{\mathbf{u}}_{i}(\mathbf{r}, t)+\sum_{i=1}^{3} \sum_{j=1}^{3} \boldsymbol{\sigma}_{i j}(\mathbf{r}, t) \frac{\partial \dot{\mathbf{u}}_{i}(\mathbf{r}, t)}{\partial \mathbf{r}_{j}}\right) d V \\
& =\int_{V}\left(\sum_{i=1}^{3} \dot{\mathbf{u}}_{i}(\mathbf{r}, t)\left(\mathbf{f}_{i}(\mathbf{r}, t)+\sum_{j=1}^{3} \frac{\partial \boldsymbol{\sigma}_{i j}(\mathbf{r}, t)}{\partial \mathbf{r}_{j}}\right)+\sum_{i=1}^{3} \sum_{j=1}^{3} \boldsymbol{\sigma}_{i j}(\mathbf{r}, t) \frac{\partial \dot{\mathbf{u}}_{i}(\mathbf{r}, t)}{\partial \mathbf{r}_{j}}\right) d V \\
& =\int_{V}\left(\sum_{i=1}^{3} \dot{\mathbf{u}}_{i}(\mathbf{r}, t) \rho(\mathbf{r}) \ddot{\mathbf{u}}_{i}(\mathbf{r}, t)+\sum_{i=1}^{3} \sum_{j=1}^{3} \boldsymbol{\sigma}_{i j}(\mathbf{r}, t) \frac{\partial \dot{\mathbf{u}}_{i}(\mathbf{r}, t)}{\partial \mathbf{r}_{j}}\right) d V \\
& =\frac{\partial}{\partial t} \int_{V} \sum_{i=1}^{3} \frac{1}{2} \rho(\mathbf{r}) \dot{\mathbf{u}}_{i}^{2}(\mathbf{r}, t) d V+\int_{V} \sum_{i=1}^{3} \sum_{j=1}^{3} \boldsymbol{\sigma}_{i j}(\mathbf{r}, t) \frac{\partial \dot{\mathbf{u}}_{i}(\mathbf{r}, t)}{\partial \mathbf{r}_{j}} d V \\
& =\frac{\partial}{\partial t} \int_{V} \sum_{i=1}^{3} \frac{1}{2} \rho(\mathbf{r}) \dot{\mathbf{u}}_{i}^{2}(\mathbf{r}, t) d V+\int_{V} \sum_{i=1}^{3} \sum_{j=1}^{3} \boldsymbol{\sigma}_{i j}(\mathbf{r}, t) \dot{\boldsymbol{\epsilon}}_{i j}(\mathbf{r}, t) d V,
\end{aligned}
$$

where the step 3 is due to the Gauchy's Stress Theorem 44, step 4 is due to the Divergence Theorem, step 7 is due to the equation of motion (17) and last step is due to the following equalities:

$$
\begin{gathered}
\sum_{i=1}^{3} \sum_{j=1}^{3} \boldsymbol{\sigma}_{i j}(\mathbf{r}, t) \dot{\boldsymbol{\epsilon}}_{i j}(\mathbf{r}, t) \\
\text { Eq. (8) } \Rightarrow=\frac{1}{2} \sum_{i=1}^{3} \sum_{j=1}^{3} \boldsymbol{\sigma}_{i j}(\mathbf{r}, t)\left(\frac{\partial \dot{\mathbf{u}}_{i}(\mathbf{r}, t)}{\partial \mathbf{r}_{j}}+\frac{\partial \dot{\mathbf{u}}_{j}(\mathbf{r}, t)}{\partial \mathbf{r}_{i}}\right) \\
\boldsymbol{\sigma}_{i j}=\boldsymbol{\sigma}_{j i} \Rightarrow=\frac{1}{2}\left(\sum_{i=1}^{3} \sum_{j=1}^{3} \boldsymbol{\sigma}_{i j}(\mathbf{r}, t) \frac{\partial \dot{\mathbf{u}}_{i}(\mathbf{r}, t)}{\partial \mathbf{r}_{j}}+\sum_{i=1}^{3} \sum_{j=1}^{3} \boldsymbol{\sigma}_{j i}(\mathbf{r}, t) \frac{\partial \dot{\mathbf{u}}_{j}(\mathbf{r}, t)}{\partial \mathbf{r}_{i}}\right) \\
=\sum_{i=1}^{3} \sum_{j=1}^{3} \boldsymbol{\sigma}_{i j}(\mathbf{r}, t) \frac{\partial \dot{\mathbf{u}}_{i}(\mathbf{r}, t)}{\partial \mathbf{r}_{j}} .
\end{gathered}
$$

Theorem 11.3 (Uniqueness of Displacement Fields in Elastodynamics) Assume there is an elastic medium with volume $V$ and surface $S=S_{1} \cup S_{2}$ and let $\left[T_{0}, T_{1}\right]$ be a time interval. Suppose that

$$
\begin{aligned}
& \mathbf{u}^{(1)} \in \mathcal{E}\left(\varrho, \mathbf{c}, \mathbf{f}, V \times S \times\left[T_{0}, T_{1}\right]\right), \\
& \mathbf{u}^{(2)} \in \mathcal{E}\left(\varrho, \mathbf{c}, \mathbf{f}, V \times S \times\left[T_{0}, T_{1}\right]\right)
\end{aligned}
$$

are two displacement fields under the initial conditions

$$
\begin{array}{ll}
\mathbf{u}^{(1)}\left(\mathbf{r}, T_{0}\right)=\mathbf{u}^{(2)}\left(\mathbf{r}, T_{0}\right) & \forall \mathbf{r} \in V \\
\dot{\mathbf{u}}^{(1)}\left(\mathbf{r}, T_{0}\right)=\dot{\mathbf{u}}^{(2)}\left(\mathbf{r}, T_{0}\right) & \forall \mathbf{r} \in V
\end{array}
$$

and boundary conditions

$$
\begin{aligned}
& \mathbf{u}^{(1)}(\mathbf{r}, t)=\mathbf{u}^{(2)}(\mathbf{r}, t) \quad \forall \mathbf{r} \in S_{1} \quad \text { and } \quad \forall t \in\left[T_{0}, T_{1}\right], \\
& \mathbf{t}\left(\mathbf{r}, \mathbf{n}(\mathbf{r}), t, \mathbf{u}^{(1)}\right)=\mathbf{t}\left(\mathbf{r}, \mathbf{n}(\mathbf{r}), t, \mathbf{u}^{(2)}\right) \quad \forall \mathbf{r} \in S_{2} \quad \text { and } \quad \forall t \in\left[T_{0}, T_{1}\right] \text {. }
\end{aligned}
$$


Then we have

$$
\mathbf{u}^{(1)}=\mathbf{u}^{(2)}
$$

if the elastic tensor field $\mathbf{c}$ is positive-definite.

Proof Let displacement field $\mathbf{u}=\mathbf{u}^{(1)}-\mathbf{u}^{(2)}$. Then we have from the Principle of Superposition 10.1 that

$$
\mathbf{u} \in \mathcal{E}\left(\varrho, \mathbf{c}, \mathbf{0}, V \times S \times\left[T_{0}, T_{1}\right]\right)
$$

under the initial conditions

$$
\mathbf{u}\left(\mathbf{r}, T_{0}\right)=\dot{\mathbf{u}}\left(\mathbf{r}, T_{0}\right)=\mathbf{0} \quad \forall \mathbf{r} \in V
$$

and boundary conditions

$$
\begin{array}{rlll}
\mathbf{u}(\mathbf{r}, t)=\mathbf{0} & \forall \mathbf{r} \in S_{1} & \text { and } & \forall t \in\left[T_{0}, T_{1}\right] \\
\mathbf{t}(\mathbf{r}, \mathbf{n}(\mathbf{r}), t, \mathbf{u})=\mathbf{0} & \forall \mathbf{r} \in S_{2} & \text { and } & \forall t \in\left[T_{0}, T_{1}\right] .
\end{array}
$$

Now consider the rate of mechanical work done on the medium, we know from equation (29) that

$$
R_{W}(\mathbf{r}, t)=\int_{V} \mathbf{f}(\mathbf{r}, t) \cdot \dot{\mathbf{u}}(\mathbf{r}, t) d V+\int_{S} \mathbf{t}(\mathbf{r}, \mathbf{n}(\mathbf{r}), t, \mathbf{u}) \cdot \dot{\mathbf{u}}(\mathbf{r}, t) d S .
$$

Since the volume force field $\mathbf{f}$ causing the displacement field $\mathbf{u}$ is zero and due to the boundary conditions, we have zero rate of mechanical work at all positions $\mathbf{r} \in V$ and time $t \in\left[T_{0}, T_{1}\right]$, i.e.,

$$
R_{W}(\mathbf{r}, t)=0 \quad \forall \mathbf{r} \in V \quad \text { and } \quad \forall t \in\left[T_{0}, T_{1}\right]
$$

Following the equation $(30)$, we then have

$$
\frac{\partial}{\partial t} \int_{V} \sum_{i=1}^{3} \frac{1}{2} \rho(\mathbf{r}) \dot{\mathbf{u}}_{i}^{2}(\mathbf{r}, t) d V+\int_{V} \sum_{i=1}^{3} \sum_{j=1}^{3} \boldsymbol{\sigma}_{i j}(\mathbf{r}, t) \dot{\boldsymbol{\epsilon}}_{i j}(\mathbf{r}, t) d V=0 \quad \forall \mathbf{r} \in V \quad \text { and } \quad \forall t \in\left[T_{0}, T_{1}\right] .
$$

Integrating both sides of equation respect to $t$ from $T_{0}$ to $\tau \in\left[T_{0}, T_{1}\right]$, we obtain

$$
\int_{T_{0}}^{\tau}\left(\frac{\partial}{\partial t} \int_{V} \sum_{i=1}^{3} \frac{1}{2} \rho(\mathbf{r}) \dot{\mathbf{u}}_{i}^{2}(\mathbf{r}, t) d V\right) d t+\int_{T_{0}}^{\tau}\left(\int_{V} \sum_{i=1}^{3} \sum_{j=1}^{3} \boldsymbol{\sigma}_{i j}(\mathbf{r}, t) \dot{\boldsymbol{\epsilon}}_{i j}(\mathbf{r}, t) d V\right) d t=b,
$$

which is a constant for all $\mathbf{r} \in V$ and $t \in\left[T_{0}, T_{1}\right]$. Note that the first integral of equation (32) gives

$$
\begin{aligned}
& \int_{T_{0}}^{\tau}\left(\frac{\partial}{\partial t} \int_{V} \sum_{i=1}^{3} \frac{1}{2} \rho(\mathbf{r}) \dot{\mathbf{u}}_{i}^{2}(\mathbf{r}, t) d V\right) d t \\
= & \left.\int_{V} \sum_{i=1}^{3} \frac{1}{2} \rho(\mathbf{r}) \dot{\mathbf{u}}_{i}^{2}(\mathbf{r}, t) d V\right|_{t=T_{0}} ^{\tau} \\
= & \int_{V} \sum_{i=1}^{3} \frac{1}{2} \rho(\mathbf{r}) \dot{\mathbf{u}}_{i}^{2}(\mathbf{r}, \tau) d V-\int_{V} \sum_{i=1}^{3} \frac{1}{2} \rho(\mathbf{r}) \dot{\mathbf{u}}_{i}^{2}\left(\mathbf{r}, T_{0}\right) d V \\
= & \int_{V} \sum_{i=1}^{3} \frac{1}{2} \rho(\mathbf{r}) \dot{\mathbf{u}}_{i}^{2}(\mathbf{r}, \tau) d V,
\end{aligned}
$$

where the last equality follows that the initial velocity is zero anywhere.

We now simplify the second integral of the equation (32). We first interchange the order of integrals:

$$
\begin{aligned}
& \int_{T_{0}}^{\tau}\left(\int_{V} \sum_{i=1}^{3} \sum_{j=1}^{3} \boldsymbol{\sigma}_{i j}(\mathbf{r}, t) \dot{\boldsymbol{\epsilon}}_{i j}(\mathbf{r}, t) d V\right) d t \\
= & \int_{V}\left(\int_{T_{0}}^{\tau} \sum_{i=1}^{3} \sum_{j=1}^{3} \boldsymbol{\sigma}_{i j}(\mathbf{r}, t) \dot{\boldsymbol{\epsilon}}_{i j}(\mathbf{r}, t) d t\right) d V
\end{aligned}
$$


We then try to solve the integral with respect to time in the braclets:

$$
\begin{aligned}
& \int_{T_{0}}^{\tau} \sum_{i=1}^{3} \sum_{j=1}^{3} \boldsymbol{\sigma}_{i j}(\mathbf{r}, t) \dot{\boldsymbol{\epsilon}}_{i j}(\mathbf{r}, t) d t \\
= & \sum_{i=1}^{3} \sum_{j=1}^{3} \int_{T_{0}}^{\tau} \boldsymbol{\sigma}_{i j}(\mathbf{r}, t) \dot{\boldsymbol{\epsilon}}_{i j}(\mathbf{r}, t) d t \\
= & \sum_{i=1}^{3} \sum_{j=1}^{3}\left(\left.\boldsymbol{\sigma}_{i j}(\mathbf{r}, t) \boldsymbol{\epsilon}_{i j}(\mathbf{r}, t)\right|_{t=T_{0}} ^{\tau}-\int_{T_{0}}^{\tau} \dot{\boldsymbol{\sigma}}_{i j}(\mathbf{r}, t) \boldsymbol{\epsilon}_{i j}(\mathbf{r}, t) d t\right) \\
= & \sum_{i=1}^{3} \sum_{j=1}^{3}\left(\left.\sum_{k=1}^{3} \sum_{l=1}^{3} \mathbf{c}_{i j k l}(\mathbf{r}) \boldsymbol{\epsilon}_{k l}(\mathbf{r}, t) \boldsymbol{\epsilon}_{i j}(\mathbf{r}, t)\right|_{t=T_{0}} ^{\tau}-\int_{T_{0}}^{\tau} \sum_{k=1}^{3} \sum_{l=1}^{3} \mathbf{c}_{i j k l}(\mathbf{r}) \dot{\boldsymbol{\epsilon}}_{k l}(\mathbf{r}, t) \boldsymbol{\epsilon}_{i j}(\mathbf{r}, t) d t\right) \\
= & \left.\sum_{i=1}^{3} \sum_{j=1}^{3} \sum_{k=1}^{3} \sum_{l=1}^{3} \mathbf{c}_{i j k l}(\mathbf{r}) \boldsymbol{\epsilon}_{k l}(\mathbf{r}, t) \boldsymbol{\epsilon}_{i j}(\mathbf{r}, t)\right|_{t=T_{0}} ^{\tau}-\int_{T_{0}}^{\tau} \sum_{i=1}^{3} \sum_{j=1}^{3} \sum_{k=1}^{3} \sum_{l=1}^{3} \mathbf{c}_{k l i j}(\mathbf{r}) \dot{\boldsymbol{\epsilon}}_{k l}(\mathbf{r}, t) \boldsymbol{\epsilon}_{i j}(\mathbf{r}, t) d t \\
= & \left.\sum_{i=1}^{3} \sum_{j=1}^{3} \sum_{k=1}^{3} \sum_{l=1}^{3} \mathbf{c}_{i j k l}(\mathbf{r}) \boldsymbol{\epsilon}_{k l}(\mathbf{r}, t) \boldsymbol{\epsilon}_{i j}(\mathbf{r}, t)\right|_{t=T_{0}} ^{\tau}-\int_{T_{0}}^{\tau} \sum_{i=1}^{3} \sum_{j=1}^{3} \sum_{k=1}^{3} \sum_{l=1}^{3} \mathbf{c}_{i j k l}(\mathbf{r}) \dot{\boldsymbol{\epsilon}}_{i j}(\mathbf{r}, t) \boldsymbol{\epsilon}_{k l}(\mathbf{r}, t) d t \\
= & \left.\sum_{i=1}^{3} \sum_{j=1}^{3} \sum_{k=1}^{3} \sum_{l=1}^{3} \mathbf{c}_{i j k l}(\mathbf{r}) \boldsymbol{\epsilon}_{k l}(\mathbf{r}, t) \boldsymbol{\epsilon}_{i j}(\mathbf{r}, t)\right|_{t=T_{0}} ^{\tau}-\int_{T_{0}}^{\tau} \sum_{i=1}^{3} \sum_{j=1}^{3} \boldsymbol{\sigma}_{i j}(\mathbf{r}, t) \dot{\boldsymbol{\epsilon}}_{i j}(\mathbf{r}, t) d t,
\end{aligned}
$$

where step 4 uses the symmetric property of elastic tensor, i.e., $\mathbf{c}_{i j k l}=\mathbf{c}_{k l i j}$ and step 5 uses the fact that swapping the index $i \longleftrightarrow k$ and $j \longleftrightarrow l$ will not change the value of the total summation.

Note that the left hand-side of equation (34) is the same with the second term on the right hand-side of the equation. Then, we obtain the time integral

$$
\begin{aligned}
& \int_{T_{0}} \sum_{i=1}^{3} \sum_{j=1}^{3} \boldsymbol{\sigma}_{i j}(\mathbf{r}, t) \dot{\boldsymbol{\epsilon}}_{i j}(\mathbf{r}, t) d t \\
= & \left.\frac{1}{2} \sum_{i=1}^{3} \sum_{j=1}^{3} \sum_{k=1}^{3} \sum_{l=1}^{3} \mathbf{c}_{i j k l}(\mathbf{r}) \boldsymbol{\epsilon}_{k l}(\mathbf{r}, t) \boldsymbol{\epsilon}_{i j}(\mathbf{r}, t)\right|_{t=T_{0}} ^{\tau} \\
= & \frac{1}{2} \sum_{i=1}^{3} \sum_{j=1}^{3} \sum_{k=1}^{3} \sum_{l=1}^{3} \mathbf{c}_{i j k l}(\mathbf{r}) \boldsymbol{\epsilon}_{k l}(\mathbf{r}, \tau) \boldsymbol{\epsilon}_{i j}(\mathbf{r}, \tau)-\frac{1}{2} \sum_{i=1}^{3} \sum_{j=1}^{3} \sum_{k=1}^{3} \sum_{l=1}^{3} \mathbf{c}_{i j k l}(\mathbf{r}) \boldsymbol{\epsilon}_{k l}\left(\mathbf{r}, T_{0}\right) \boldsymbol{\epsilon}_{i j}\left(\mathbf{r}, T_{0}\right) \\
= & \frac{1}{2} \sum_{i=1}^{3} \sum_{j=1}^{3} \sum_{k=1}^{3} \sum_{l=1}^{3} \mathbf{c}_{i j k l}(\mathbf{r}) \boldsymbol{\epsilon}_{k l}(\mathbf{r}, \tau) \boldsymbol{\epsilon}_{i j}(\mathbf{r}, \tau)-\frac{1}{2} \sum_{i=1}^{3} \sum_{j=1}^{3} \sum_{k=1}^{3} \sum_{l=1}^{3} \mathbf{c}_{i j k l}(\mathbf{r}) \frac{\partial \mathbf{u}_{k}\left(\mathbf{r}, T_{0}\right)}{\partial \mathbf{r}_{l}} \frac{\partial \mathbf{u}_{i}\left(\mathbf{r}, T_{0}\right)}{\partial \mathbf{r}_{j}} \\
= & \frac{1}{2} \sum_{i=1}^{3} \sum_{j=1}^{3} \sum_{k=1}^{3} \sum_{l=1}^{3} \mathbf{c}_{i j k l}(\mathbf{r}) \boldsymbol{\epsilon}_{k l}(\mathbf{r}, \tau) \boldsymbol{\epsilon}_{i j}(\mathbf{r}, \tau),
\end{aligned}
$$


where the last step is due to the initial condition of displacement field and the third step is due to

$$
\begin{aligned}
& \sum_{i=1}^{3} \sum_{j=1}^{3} \sum_{k=1}^{3} \sum_{l=1}^{3} \mathbf{c}_{i j k l}(\mathbf{r}) \boldsymbol{\epsilon}_{k l}\left(\mathbf{r}, T_{0}\right) \boldsymbol{\epsilon}_{i j}\left(\mathbf{r}, T_{0}\right) \\
= & \sum_{i=1}^{3} \sum_{j=1}^{3} \boldsymbol{\sigma}_{i j}\left(\mathbf{r}, T_{0}\right) \boldsymbol{\epsilon}_{i j}\left(\mathbf{r}, T_{0}\right) \\
\text { Similar to Eq. [31] } \Rightarrow \quad= & \sum_{i=1}^{3} \sum_{j=1}^{3} \boldsymbol{\sigma}_{i j}\left(\mathbf{r}, T_{0}\right) \frac{\partial \mathbf{u}_{i}\left(\mathbf{r}, T_{0}\right)}{\partial \mathbf{r}_{j}} \\
\text { Lemma 6.2 } \Rightarrow \quad= & \sum_{i=1}^{3} \sum_{j=1}^{3} \sum_{k=1}^{3} \sum_{l=1}^{3} \mathbf{c}_{i j k l}(\mathbf{r}) \frac{\partial \mathbf{u}_{k}\left(\mathbf{r}, T_{0}\right)}{\partial \mathbf{r}_{l}} \frac{\partial \mathbf{u}_{i}\left(\mathbf{r}, T_{0}\right)}{\partial \mathbf{r}_{j}} .
\end{aligned}
$$

Putting equation (35) and equation (33) back to equation (32), we obtain

$$
\int_{V} \sum_{i=1}^{3} \rho(\mathbf{r}) \dot{\mathbf{u}}_{i}^{2}(\mathbf{r}, \tau) d V+\int_{V} \sum_{i=1}^{3} \sum_{j=1}^{3} \sum_{k=1}^{3} \sum_{l=1}^{3} \mathbf{c}_{i j k l}(\mathbf{r}) \boldsymbol{\epsilon}_{k l}(\mathbf{r}, \tau) \boldsymbol{\epsilon}_{i j}(\mathbf{r}, \tau) d V=b
$$

for all $\mathbf{r} \in V$ and $\tau \in\left[T_{0}, T_{1}\right]$. Let $\tau=T_{0}$ in equation (37) then we have

$$
b=\int_{V} \sum_{i=1}^{3} \rho(\mathbf{r}) \dot{\mathbf{u}}_{i}^{2}(\mathbf{r}, \tau) d V+\int_{V} \sum_{i=1}^{3} \sum_{j=1}^{3} \sum_{k=1}^{3} \sum_{l=1}^{3} \mathbf{c}_{i j k l}(\mathbf{r}) \boldsymbol{\epsilon}_{k l}(\mathbf{r}, \tau) \boldsymbol{\epsilon}_{i j}(\mathbf{r}, \tau) d V
$$

$$
\begin{aligned}
\text { Eq. (36) } \Rightarrow & =\int_{V} \sum_{i=1}^{3} \rho(\mathbf{r}) \dot{\mathbf{u}}_{i}^{2}\left(\mathbf{r}, T_{0}\right) d V+\int_{V} \sum_{i=1}^{3} \sum_{j=1}^{3} \sum_{k=1}^{3} \sum_{l=1}^{3} \mathbf{c}_{i j k l}(\mathbf{r}) \frac{\partial \mathbf{u}_{k}\left(\mathbf{r}, T_{0}\right)}{\partial \mathbf{r}_{l}} \frac{\partial \mathbf{u}_{i}\left(\mathbf{r}, T_{0}\right)}{\partial \mathbf{r}_{j}} d V \\
& =0
\end{aligned}
$$

Thus, we get

$$
\int_{V} \sum_{i=1}^{3} \rho(\mathbf{r}) \dot{\mathbf{u}}_{i}^{2}(\mathbf{r}, \tau) d V+\int_{V} \sum_{i=1}^{3} \sum_{j=1}^{3} \sum_{k=1}^{3} \sum_{l=1}^{3} \mathbf{c}_{i j k l}(\mathbf{r}) \boldsymbol{\epsilon}_{k l}(\mathbf{r}, \tau) \boldsymbol{\epsilon}_{i j}(\mathbf{r}, \tau) d V=0
$$

for all $\mathbf{r} \in V$ and $\tau \in\left[T_{0}, T_{1}\right]$. Observe that the first term of equation (38) is non-negative and the second term is positive as $\mathbf{c}$ is positive-definite and strain tenor $\boldsymbol{\epsilon}(\mathbf{r}, \tau)$ is symmetric. Therefore, equation (38) implies that

$$
\begin{aligned}
\dot{\mathbf{u}}(\mathbf{r}, \tau) & =\mathbf{0}, \\
\boldsymbol{\epsilon}(\mathbf{r}, \tau) & =\mathbf{0}
\end{aligned}
$$

for all $\mathbf{r} \in V$ and $\tau \in\left[T_{0}, T_{1}\right]$. Equation (39) indicates that $\mathbf{u}(\mathbf{r}, \tau)$ is a constant for all $\mathbf{r} \in V$ and $\tau \in\left[T_{0}, T_{1}\right]$. Due to the initial condition that $\mathbf{u}\left(\mathbf{r}, T_{0}\right)=0$ for all $\mathbf{r} \in V$, we conclude that

$$
\mathbf{u}(\mathbf{r}, \tau)=\mathbf{0} \quad \forall \mathbf{r} \in V \quad \text { and } \quad \forall \tau \in\left[T_{0}, T_{1}\right]
$$

which proves the theorem.

The proof shows that the displacement field in elastodynamic case is unique under displacement boundary condition, traction boundary condition as well as mixed boundary condition. In elastostatic case, however, this is not the case. In elastostatics, we denote the displacement field set by

$$
\mathcal{E}(\mathbf{c}, \mathbf{f}, S \times V)
$$

which implies that the elastostatic displacement fields do not depend on density field and time. This is evidenced by elastostatic equation of motion 27. 
Theorem 11.4 (Uniqueness of Displacement Fields in Elastostatics) Assume there is an elastic medium with volume $V$ and surface $S=S_{1} \cup S_{2}$. Suppose that

$$
\begin{aligned}
& \mathbf{u}^{(1)} \in \mathcal{E}(\mathbf{c}, \mathbf{f}, V \times S), \\
& \mathbf{u}^{(2)} \in \mathcal{E}(\mathbf{c}, \mathbf{f}, V \times S)
\end{aligned}
$$

are two displacement fields under three different types of boundary conditions:

- Displacement boundary condition, i.e.,

$$
\mathbf{u}^{(1)}(\mathbf{r})=\mathbf{u}^{(2)}(\mathbf{r}) \quad \forall \mathbf{r} \in S
$$

- Traction boundary condition, i.e.,

$$
\mathbf{t}\left(\mathbf{r}, \mathbf{n}(\mathbf{r}), \mathbf{u}^{(1)}\right)=\mathbf{t}\left(\mathbf{r}, \mathbf{n}(\mathbf{r}), \mathbf{u}^{(2)}\right) \quad \forall \mathbf{r} \in S
$$

- Strictly mixed boundary condition, i.e.,

$$
\begin{aligned}
\mathbf{u}^{(1)}(\mathbf{r}) & =\mathbf{u}^{(2)}(\mathbf{r}) & & \forall \mathbf{r} \in S_{1} \neq \emptyset \\
\mathbf{t}\left(\mathbf{r}, \mathbf{n}(\mathbf{r}), \mathbf{u}^{(1)}\right) & =\mathbf{t}\left(\mathbf{r}, \mathbf{n}(\mathbf{r}), \mathbf{u}^{(2)}\right) & & \forall \mathbf{r} \in S_{2} \neq \emptyset
\end{aligned}
$$

Then, if the elastic tensor field $\mathbf{c}$ is positive-definite, we have

- For displacement boundary condition,

$$
\mathbf{u}^{(1)}=\mathbf{u}^{(2)}
$$

- For traction boundary condition,

$$
\mathbf{u}^{(1)} \neq \mathbf{u}^{(2)}
$$

- For strictly mixed boundary condition,

$$
\mathbf{u}^{(1)}=\mathbf{u}^{(2)}
$$

Proof Let displacement field $\mathbf{u}=\mathbf{u}^{(1)}-\mathbf{u}^{(2)}$. Then we have from the Principle of Superposition 10.1 that

$$
\mathbf{u} \in \mathcal{E}(\mathbf{c}, \mathbf{0}, V \times S)
$$

with corresponding boundary conditions given by:

- Displacement boundary condition, i.e.,

$$
\mathbf{u}(\mathbf{r})=\mathbf{0} \quad \forall \mathbf{r} \in S
$$

- Traction boundary condition, i.e.,

$$
\mathbf{t}(\mathbf{r}, \mathbf{n}(\mathbf{r}), \mathbf{u})=\mathbf{0} \quad \forall \mathbf{r} \in S
$$

- Strictly mixed boundary condition, i.e.,

$$
\begin{aligned}
\mathbf{u}(\mathbf{r})=\mathbf{0} & \forall \mathbf{r} \in S_{1} \neq \emptyset \\
\mathbf{t}(\mathbf{r}, \mathbf{n}(\mathbf{r}), \mathbf{u})=\mathbf{0} & \forall \mathbf{r} \in S_{2} \neq \emptyset .
\end{aligned}
$$


Now consider the mechanical work $W(\mathbf{r})$ at position $\mathbf{r}$ :

$$
\begin{aligned}
& W(\mathbf{r})=\int_{V} \mathbf{f}(\mathbf{r}) \cdot \mathbf{u}(\mathbf{r}) d V+\int_{S} \mathbf{t}(\mathbf{r}, \mathbf{n}(\mathbf{r}), \mathbf{u}) \cdot \mathbf{u}(\mathbf{r}) d S \\
& =\int_{V} \sum_{i=1}^{3} \mathbf{f}_{i}(\mathbf{r}) \mathbf{u}_{i}(\mathbf{r}) d V+\int_{S} \sum_{i=1}^{3} \mathbf{t}_{i}(\mathbf{r}, \mathbf{n}(\mathbf{r}), \mathbf{u}) \mathbf{u}_{i}(\mathbf{r}) d S \\
& =\int_{V} \sum_{i=1}^{3} \mathbf{f}_{i}(\mathbf{r}) \mathbf{u}_{i}(\mathbf{r}) d V+\int_{S} \sum_{i=1}^{3} \sum_{j=1}^{3} \boldsymbol{\sigma}_{i j}(\mathbf{r}) \mathbf{n}_{j}(\mathbf{r}) \mathbf{u}_{i}(\mathbf{r}) d S \\
& =\int_{V} \sum_{i=1}^{3} \mathbf{f}_{i}(\mathbf{r}) \mathbf{u}_{i}(\mathbf{r}) d V+\int_{V} \sum_{i=1}^{3} \sum_{j=1}^{3} \frac{\partial}{\partial \mathbf{r}_{j}}\left(\boldsymbol{\sigma}_{i j}(\mathbf{r}, t) \mathbf{u}_{i}(\mathbf{r})\right) d V \\
& =\int_{V}\left(\sum_{i=1}^{3} \mathbf{f}_{i}(\mathbf{r}) \mathbf{u}_{i}(\mathbf{r})+\sum_{i=1}^{3} \sum_{j=1}^{3} \frac{\partial \boldsymbol{\sigma}_{i j}(\mathbf{r})}{\partial \mathbf{r}_{j}} \mathbf{u}_{i}(\mathbf{r})+\sum_{i=1}^{3} \sum_{j=1}^{3} \boldsymbol{\sigma}_{i j}(\mathbf{r}) \frac{\partial \mathbf{u}_{i}(\mathbf{r})}{\partial \mathbf{r}_{j}}\right) d V \\
& =\int_{V}\left(\sum_{i=1}^{3} \dot{\mathbf{u}}_{i}(\mathbf{r})\left(\mathbf{f}_{i}(\mathbf{r}, t)+\sum_{j=1}^{3} \frac{\partial \boldsymbol{\sigma}_{i j}(\mathbf{r})}{\partial \mathbf{r}_{j}}\right)+\sum_{i=1}^{3} \sum_{j=1}^{3} \boldsymbol{\sigma}_{i j}(\mathbf{r}) \frac{\partial \mathbf{u}_{i}(\mathbf{r})}{\partial \mathbf{r}_{j}}\right) d V \\
& =\int_{V}\left(0+\sum_{i=1}^{3} \sum_{j=1}^{3} \boldsymbol{\sigma}_{i j}(\mathbf{r}) \frac{\partial \mathbf{u}_{i}(\mathbf{r})}{\partial \mathbf{r}_{j}}\right) d V \\
& =\int_{V} \sum_{i=1}^{3} \sum_{j=1}^{3} \sum_{k=1}^{3} \sum_{l=1}^{3} \mathbf{c}_{i j k l}(\mathbf{r}) \frac{\partial \mathbf{u}_{k}(\mathbf{r})}{\partial \mathbf{r}_{l}} \frac{\partial \mathbf{u}_{i}(\mathbf{r})}{\partial \mathbf{r}_{j}} d V \\
& =\int_{V} \sum_{i=1}^{3} \sum_{j=1}^{3} \sum_{k=1}^{3} \sum_{l=1}^{3} \mathbf{c}_{i j k l}(\mathbf{r}) \boldsymbol{\epsilon}_{k l}(\mathbf{r}) \boldsymbol{\epsilon}_{i j}(\mathbf{r}) d V
\end{aligned}
$$

where the third equality uses the Gauchy's Stress Theorem (4), the forth equality uses the Divergence Theorem, the seventh equality uses the equation of motion 17 by taking $\ddot{\mathbf{u}}(\mathbf{r}, t)=\mathbf{0}$ due to time-independence and the last step uses equation (36).

Since the volume force field $\mathbf{f}$ causing the displacement field $\mathbf{u}$ is zero and due to the boundary conditions, we have from equation (44) that the mechanical work is zero at all positions $\mathbf{r} \in V$, i.e.,

$$
W(\mathbf{r})=0 \quad \forall \mathbf{r} \in V .
$$

Thus, we have from equation (45) that

$$
\int_{V} \sum_{i=1}^{3} \sum_{j=1}^{3} \sum_{k=1}^{3} \sum_{l=1}^{3} \mathbf{c}_{i j k l}(\mathbf{r}) \boldsymbol{\epsilon}_{k l}(\mathbf{r}) \boldsymbol{\epsilon}_{i j}(\mathbf{r}) d V=0 \quad \forall \mathbf{r} \in V .
$$

Since the elastic tensor field $\mathbf{c}$ is positive-definite, equation 46 implies that

$$
\boldsymbol{\epsilon}(\mathbf{r})=\mathbf{0} \quad \forall \mathbf{r} \in V \text {. }
$$

It can be verified that the strain tensor $\epsilon(\mathbf{r})$ in expression (2) and rotation tensor $\Omega(\mathbf{r})$ in expression (3) have the following identity:

$$
\nabla \times \boldsymbol{\epsilon}(\mathbf{r})^{\top}=\nabla \boldsymbol{\omega}(\mathbf{r}) \quad \forall \mathbf{r} \in V
$$

where

- $\nabla \times \boldsymbol{\epsilon}(\mathbf{r})^{\top}$ is the curl of $\boldsymbol{\epsilon}(\mathbf{r})^{\top}$;

- $\nabla \boldsymbol{\omega}(\mathbf{r})$ is the gradient of vector $\boldsymbol{\omega}(\mathbf{r})$;

- $\boldsymbol{\omega}(\mathbf{r})=\left(\boldsymbol{\Omega}_{32}(\mathbf{r}), \boldsymbol{\Omega}_{13}(\mathbf{r}), \boldsymbol{\Omega}_{21}(\mathbf{r})\right)^{\top}$. 
As the strain tensor $\epsilon(\mathbf{r})$ is zero at all $\mathbf{r} \in V$ (see equation (47)), we have from indentity (48) that

$$
\nabla \boldsymbol{\omega}(\mathbf{r})=\mathbf{0} \quad \forall \mathbf{r} \in V
$$

Then the equality (49) implies that $\boldsymbol{\omega}(\mathbf{r})$ is constant for all $\mathbf{r} \in V$. Note that vector $\boldsymbol{\omega}(\mathbf{r})$ uniquely determines the rotation tensor $\Omega(\mathbf{r})$, we obtain that

$$
\Omega(\mathbf{r})=\mathbf{D},
$$

a $3 \times 3$ constant matrix for all $\mathbf{r} \in V$. We learned from Section 2 that the Jacobian matrix $\mathbf{J}(\mathbf{r})$ of displacement $\mathbf{u}(\mathbf{r})$ has the following relation with strain tensor and rotation tensor:

$$
\mathbf{J}(\mathbf{r})=\nabla \mathbf{u}(\mathbf{r})=\boldsymbol{\epsilon}(\mathbf{r})+\mathbf{\Omega}(\mathbf{r}) \quad \forall \mathbf{r} \in V
$$

Using zero strain field (47), equation (51) gives

$$
\mathbf{\Omega}(\mathbf{r})=\nabla \mathbf{u}(\mathbf{r}) \quad \forall \mathbf{r} \in V .
$$

Together with equation (50), equation (52) implies that

$$
\nabla \mathbf{u}(\mathbf{r})=\mathbf{D} \quad \forall \mathbf{r} \in V
$$

which consequently gives the displacement field $\mathbf{u}$ of the medium when the strain field is zero:

$$
\mathbf{u}(\mathbf{r})=\mathbf{D} \mathbf{r}+\mathbf{L} \quad \forall \mathbf{r} \in V,
$$

where $\mathbf{L} \in \mathbb{R}^{3}$ is a constant vector for all $\mathbf{r} \in V$.

Now we examine the displacement field $\mathbf{u}$ under different boundary conditions.

- Displacement boundary condition: Due to displacement boundary condition (40), equation (53) requires

$$
\mathbf{D}=\mathbf{0} \quad \text { and } \quad \mathbf{L}=\mathbf{0} \quad \forall \mathbf{r} \in S .
$$

As $\mathbf{D}$ and $\mathbf{L}$ are spatially homogeneous (i.e., constant for all $\mathbf{r} \in V$ ), we then have

$$
\mathbf{D}=\mathbf{0} \quad \text { and } \quad \mathbf{L}=\mathbf{0} \quad \forall \mathbf{r} \in V .
$$

Thus,

$$
\mathbf{u}(\mathbf{r})=\mathbf{0} \quad \forall \mathbf{r} \in V,
$$

which proves that $\mathbf{u}^{(1)}=\mathbf{u}^{(2)}$ under the displacement boundary condition.

- Traction boundary condition: Since the traction boundary condition 41 does not require the displacements to vanish on the boundary, $\mathbf{D}$ and $\mathbf{L}$ in equation (53) are not zero in general. Thus,

$$
\mathbf{u}(\mathbf{r}) \neq \mathbf{0} \quad \forall \mathbf{r} \in V
$$

which proves that $\mathbf{u}^{(1)} \neq \mathbf{u}^{(2)}$ under the traction boundary condition.

- Strictly mixed boundary condition: Although the strictly mixed boundary conditions 42, and 43, only require displacements to vanish on part of the boundary, i.e.,

$$
\mathbf{D}=\mathbf{0} \quad \text { and } \quad \mathbf{L}=\mathbf{0} \quad \forall \mathbf{r} \in S_{1},
$$

the spatial homogeneity of $\mathbf{D}$ and $\mathbf{L}$ still requires

$$
\mathbf{D}=\mathbf{0} \quad \text { and } \quad \mathbf{L}=\mathbf{0} \quad \forall \mathbf{r} \in V,
$$

which proves that $\mathbf{u}^{(1)}=\mathbf{u}^{(2)}$ under the strictly mixed boundary condition.

The uniqueness theorem 11.4 for elastostatics tells us that the displacement field is unique only under displacement and strictly mixed boundary conditions while under traction boundary condition this is generally not the case. 


\section{Reciprocity Theorem}

Theorem 12.1 (Betti's theorem) Assume there is a continuous elastic medium with volume $V$ and surface $S$. Let $\mathbf{f}(\mathbf{r}, t)$ and $\mathbf{g}(\mathbf{r}, t)$ be two volume forces acting on the volume element $d V$ at position $\mathbf{r}$ and time $t$. The corresponding displacements at position $\mathbf{r}$ and time $t$ are denoted by $\mathbf{u}(\mathbf{r}, t)$ and $\mathbf{w}(\mathbf{r}, t)$ respectively. In addition, assume that $\mathbf{t}(\mathbf{r}, \mathbf{n}(\mathbf{r}), t, \mathbf{u})$ and $\mathbf{t}(\mathbf{r}, \mathbf{n}(\mathbf{r}), t, \mathbf{w})$ are stresses (due to displacement fields $\mathbf{u}$ and $\mathbf{w}$ respectively) acting on the surface element $d S$ (with unit normal vector $\mathbf{n}(\mathbf{r})$ ) at position $\mathbf{r}$ and time $t$. The initial condition and boundary conditions of $\mathbf{u}(\mathbf{r}, t)$ are generally different from those of $\mathbf{w}(\mathbf{r}, t)$. Then the following equality holds:

$$
\begin{aligned}
\int_{V} \sum_{i=1}^{3}\left(\mathbf{f}_{i}(\mathbf{r}, t)-\right. & \left.\rho(\mathbf{r}) \ddot{\mathbf{u}}_{i}(\mathbf{r}, t)\right) \mathbf{w}_{i}(\mathbf{r}, t) d V+\int_{S} \sum_{i=1}^{3} \mathbf{t}_{i}(\mathbf{r}, \mathbf{n}(\mathbf{r}), t, \mathbf{u}) \mathbf{w}_{i}(\mathbf{r}, t) d S \\
& =\int_{V} \sum_{i=1}^{3}\left(\mathbf{g}_{i}(\mathbf{r}, t)-\rho(\mathbf{r}) \ddot{\mathbf{w}}_{i}(\mathbf{r}, t)\right) \mathbf{u}_{i}(\mathbf{r}, t) d V+\int_{S} \sum_{i=1}^{3} \mathbf{t}_{i}(\mathbf{r}, \mathbf{n}(\mathbf{r}), t, \mathbf{w}) \mathbf{u}_{i}(\mathbf{r}, t) d S .
\end{aligned}
$$

Proof We prove the theorem from the left-hand side of the equality. According to the equation of motion 17 for an infinite medium, the first term on the left of the equality becomes:

$$
\int_{V} \sum_{i=1}^{3}\left(\mathbf{f}_{i}(\mathbf{r}, t)-\rho(\mathbf{r}) \ddot{\mathbf{u}}_{i}(\mathbf{r}, t)\right) \mathbf{w}_{i}(\mathbf{r}, t) d V=-\int_{V} \sum_{i=1}^{3} \sum_{j=1}^{3} \frac{\partial \boldsymbol{\sigma}_{i j}(\mathbf{r}, t)}{\partial \mathbf{r}_{j}} \mathbf{w}_{i}(\mathbf{r}, t) d V .
$$

Due to the Gauchy's Stress Theorem 4 and Divergence Theorem, we have for the second term on the left-hand side of the equality:

$$
\begin{aligned}
& \int_{S} \sum_{i=1}^{3} \mathbf{t}_{i}(\mathbf{r}, \mathbf{n}(\mathbf{r}), t, \mathbf{u}) \mathbf{w}_{i}(\mathbf{r}, t) d S \\
= & \int_{S} \sum_{i=1}^{3} \sum_{j=1}^{3} \boldsymbol{\sigma}_{i j}(\mathbf{r}, t, \mathbf{u}) \mathbf{n}_{j}(\mathbf{r}) \mathbf{w}_{i}(\mathbf{r}, t) d S \\
= & \int_{V} \sum_{i=1}^{3} \sum_{j=1}^{3} \frac{\partial}{\partial \mathbf{r}_{j}}\left(\boldsymbol{\sigma}_{i j}(\mathbf{r}, t, \mathbf{u}) \mathbf{w}_{i}(\mathbf{r}, t)\right) d V \\
= & \int_{V} \sum_{i=1}^{3} \sum_{j=1}^{3} \frac{\partial \boldsymbol{\sigma}_{i j}(\mathbf{r}, t, \mathbf{u})}{\partial \mathbf{r}_{j}} \mathbf{w}_{i}(\mathbf{r}, t) d V+\int_{V} \sum_{i=1}^{3} \sum_{j=1}^{3} \boldsymbol{\sigma}_{i j}(\mathbf{r}, t, \mathbf{u}) \frac{\partial \mathbf{w}_{i}(\mathbf{r}, t)}{\partial \mathbf{r}_{j}} d V
\end{aligned}
$$

Replacing the left-hand side of equation (54) by equation (55) and (56), we have

$$
\begin{aligned}
L H S & =\int_{V} \sum_{i=1}^{3} \sum_{j=1}^{3} \boldsymbol{\sigma}_{i j}(\mathbf{r}, t, \mathbf{u}) \frac{\partial \mathbf{w}_{i}(\mathbf{r}, t)}{\partial \mathbf{r}_{j}} d V \\
& =\int_{V} \sum_{i=1}^{3} \sum_{j=1}^{3} \sum_{k=1}^{3} \sum_{l=1}^{3} \mathbf{c}_{i j k l}(\mathbf{r}) \frac{\partial \mathbf{u}_{k}(\mathbf{r}, t)}{\partial \mathbf{r}_{l}} \frac{\partial \mathbf{w}_{i}(\mathbf{r}, t)}{\partial \mathbf{r}_{j}} d V
\end{aligned}
$$

Similarly, it can be shown that the right-hand side of the equation (54) is given by

$$
\begin{aligned}
R H S & =\int_{V} \sum_{i=1}^{3} \sum_{j=1}^{3} \sum_{k=1}^{3} \sum_{l=1}^{3} \mathbf{c}_{i j k l}(\mathbf{r}) \frac{\partial \mathbf{w}_{k}(\mathbf{r}, t)}{\partial \mathbf{r}_{l}} \frac{\partial \mathbf{u}_{i}(\mathbf{r}, t)}{\partial \mathbf{r}_{j}} d V \\
& =\int_{V} \sum_{i=1}^{3} \sum_{j=1}^{3} \sum_{k=1}^{3} \sum_{l=1}^{3} \mathbf{c}_{k l i j}(\mathbf{r}) \frac{\partial \mathbf{w}_{k}(\mathbf{r}, t)}{\partial \mathbf{r}_{l}} \frac{\partial \mathbf{u}_{i}(\mathbf{r}, t)}{\partial \mathbf{r}_{j}} d V,
\end{aligned}
$$

where we use the symmetric properties of the elastic tensor (see Proposition 6.1) in the last step. Note that equation (57) is in fact the same with equation [58, thus we have

$$
L H S=R H S,
$$

which proves the theorem. 
It is important to note that Betti's Theorem does not depend on the initial conditions of displacement. It is valid for all $t \in \mathbb{R}$. In addition, it can be shown that the theorem is still valid when $\mathbf{f}(\mathbf{r}, t), \ddot{\mathbf{u}}_{i}(\mathbf{r}, t), \mathbf{t}(\mathbf{r}, \mathbf{n}(\mathbf{r}), t, \mathbf{u})$ and $\mathbf{u}(\mathbf{r}, t)$ are evaluated at $t=t_{1}$ while $\mathbf{g}(\mathbf{r}, t), \ddot{\mathbf{w}}_{i}(\mathbf{r}, t), \mathbf{t}(\mathbf{r}, \mathbf{n}(\mathbf{r}), t, \mathbf{w})$ and $\mathbf{w}(\mathbf{r}, t)$ are evaluated at $t=t_{2} \neq t_{1}$, i.e.,

$$
\begin{gathered}
\int_{V} \sum_{i=1}^{3}\left(\mathbf{f}_{i}\left(\mathbf{r}, t_{1}\right)-\rho(\mathbf{r}) \ddot{\mathbf{u}}_{i}\left(\mathbf{r}, t_{1}\right)\right) \mathbf{w}_{i}\left(\mathbf{r}, t_{2}\right) d V+\int_{S} \sum_{i=1}^{3} \mathbf{t}_{i}\left(\mathbf{r}, \mathbf{n}(\mathbf{r}), t_{1}, \mathbf{u}\right) \mathbf{w}_{i}\left(\mathbf{r}, t_{2}\right) d S \\
=\int_{V} \sum_{i=1}^{3}\left(\mathbf{g}_{i}\left(\mathbf{r}, t_{2}\right)-\rho(\mathbf{r}) \ddot{\mathbf{w}}_{i}\left(\mathbf{r}, t_{2}\right)\right) \mathbf{u}_{i}\left(\mathbf{r}, t_{1}\right) d V+\int_{S} \sum_{i=1}^{3} \mathbf{t}_{i}\left(\mathbf{r}, \mathbf{n}(\mathbf{r}), t_{2}, \mathbf{w}\right) \mathbf{u}_{i}\left(\mathbf{r}, t_{1}\right) d S
\end{gathered}
$$

Theorem 12.2 (Reciprocity Theorem) Assume that the Causality Principle (Udias et al. 2014) that the medium keeps rested until a given time $t=\tau_{0}$ when the motion starts is satisfied:

$$
\begin{aligned}
\mathbf{u}_{i}(\mathbf{r}, t) & =\dot{\mathbf{u}}_{i}(\mathbf{r}, t)=0, \quad t \leq \tau_{0} \\
\mathbf{w}_{i}(\mathbf{r}, t) & =\dot{\mathbf{w}}_{i}(\mathbf{r}, t)=0, \quad t \leq \tau_{0}
\end{aligned}
$$

for all $i \in\{1,2,3\}$ and $\mathbf{r}$ in the continuous elastic medium with volume $V$ and surface $S$. Then, we have

$$
\begin{aligned}
\int_{-\infty}^{\infty} d t \int_{V} \sum_{i=1}^{3}\left(\mathbf{u}_{i}(\mathbf{r}, t) \mathbf{g}_{i}(\mathbf{r}, \tau-t)-\mathbf{w}_{i}(\mathbf{r}, \tau-t) \mathbf{f}_{i}(\mathbf{r}, t)\right) d V \\
\quad=\int_{-\infty}^{\infty} d t \int_{S} \sum_{i=1}^{3}\left(\mathbf{w}_{i}(\mathbf{r}, \tau-t) \mathbf{t}_{i}(\mathbf{r}, \mathbf{n}(\mathbf{r}), t, \mathbf{u})-\mathbf{u}_{i}(\mathbf{r}, t) \mathbf{t}_{i}(\mathbf{r}, \mathbf{n}(\mathbf{r}), \tau-t, \mathbf{w})\right) d S
\end{aligned}
$$

for some $\tau \in \mathbb{R}$.

Proof Rearranging equation (59), we obtain

$$
\begin{array}{r}
\int_{V} \sum_{i=1}^{3} \rho(\mathbf{r})\left(\ddot{\mathbf{u}}_{i}\left(\mathbf{r}, t_{1}\right) \mathbf{w}_{i}\left(\mathbf{r}, t_{2}\right)-\ddot{\mathbf{w}}_{i}\left(\mathbf{r}, t_{2}\right) \mathbf{u}_{i}\left(\mathbf{r}, t_{1}\right)\right) d V+\int_{V} \sum_{i=1}^{3}\left(\mathbf{u}_{i}\left(\mathbf{r}, t_{1}\right) \mathbf{g}_{i}\left(\mathbf{r}, t_{2}\right)-\mathbf{w}_{i}\left(\mathbf{r}, t_{2}\right) \mathbf{f}_{i}\left(\mathbf{r}, t_{1}\right)\right) d V \\
=\int_{S} \sum_{i=1}^{3}\left(\mathbf{w}_{i}\left(\mathbf{r}, t_{2}\right) \mathbf{t}_{i}\left(\mathbf{r}, \mathbf{n}(\mathbf{r}), t_{1}, \mathbf{u}\right)-\mathbf{u}_{i}\left(\mathbf{r}, t_{1}\right) \mathbf{t}_{i}\left(\mathbf{r}, \mathbf{n}(\mathbf{r}), t_{2}, \mathbf{w}\right)\right) d S
\end{array}
$$

Let $t_{1}=t$ and $t_{2}=\tau-t$ and integrate each term in equation 63 with respect to $t$ through all time (i.e., from $-\infty$ to $\infty)$ :

$$
\begin{aligned}
\int_{-\infty}^{\infty} d t \int_{V} \sum_{i=1}^{3} \rho(\mathbf{r}) & \left(\ddot{\mathbf{u}}_{i}(\mathbf{r}, t) \mathbf{w}_{i}(\mathbf{r}, \tau-t)-\ddot{\mathbf{w}}_{i}(\mathbf{r}, \tau-t) \mathbf{u}_{i}(\mathbf{r}, t)\right) d V \\
& +\int_{-\infty}^{\infty} d t \int_{V} \sum_{i=1}^{3}\left(\mathbf{u}_{i}(\mathbf{r}, t) \mathbf{g}_{i}(\mathbf{r}, \tau-t)-\mathbf{w}_{i}(\mathbf{r}, \tau-t) \mathbf{f}_{i}(\mathbf{r}, t)\right) d V \\
= & \int_{-\infty}^{\infty} d t \int_{S} \sum_{i=1}^{3}\left(\mathbf{w}_{i}(\mathbf{r}, \tau-t) \mathbf{t}_{i}(\mathbf{r}, \mathbf{n}(\mathbf{r}), t, \mathbf{u})-\mathbf{u}_{i}(\mathbf{r}, t) \mathbf{t}_{i}(\mathbf{r}, \mathbf{n}(\mathbf{r}), \tau-t, \mathbf{w})\right) d S
\end{aligned}
$$

Then it remains to prove that the first term in equation 64 is equal to zero. We interchange the order of integrals in the first term of equation (64), which gives

$$
\begin{aligned}
& \int_{-\infty}^{\infty} d t \int_{V} \sum_{i=1}^{3} \rho(\mathbf{r})\left(\ddot{\mathbf{u}}_{i}(\mathbf{r}, t) \mathbf{w}_{i}(\mathbf{r}, \tau-t)-\ddot{\mathbf{w}}_{i}(\mathbf{r}, \tau-t) \mathbf{u}_{i}(\mathbf{r}, t)\right) d V \\
= & \int_{V} d V \int_{-\infty}^{\infty} \sum_{i=1}^{3} \rho(\mathbf{r})\left(\ddot{\mathbf{u}}_{i}(\mathbf{r}, t) \mathbf{w}_{i}(\mathbf{r}, \tau-t)-\ddot{\mathbf{w}}_{i}(\mathbf{r}, \tau-t) \mathbf{u}_{i}(\mathbf{r}, t)\right) d t
\end{aligned}
$$


Due to the causality conditions 60 and 61 , the time integral in equation 65 becomes

$$
\begin{aligned}
& \int_{-\infty}^{\infty} \sum_{i=1}^{3} \rho(\mathbf{r})\left(\ddot{\mathbf{u}}_{i}(\mathbf{r}, t) \mathbf{w}_{i}(\mathbf{r}, \tau-t)-\ddot{\mathbf{w}}_{i}(\mathbf{r}, \tau-t) \mathbf{u}_{i}(\mathbf{r}, t)\right) d t \\
= & \int_{\tau_{0}}^{\tau-\tau_{0}} \sum_{i=1}^{3} \rho(\mathbf{r}) \frac{\partial}{\partial t}\left(\dot{\mathbf{u}}_{i}(\mathbf{r}, t) \mathbf{w}_{i}(\mathbf{r}, \tau-t)+\dot{\mathbf{w}}_{i}(\mathbf{r}, \tau-t) \mathbf{u}_{i}(\mathbf{r}, t)\right) d t \\
= & \sum_{i=1}^{3} \rho(\mathbf{r}) \int_{\tau_{0}}^{\tau-\tau_{0}} \frac{\partial}{\partial t}\left(\dot{\mathbf{u}}_{i}(\mathbf{r}, t) \mathbf{w}_{i}(\mathbf{r}, \tau-t)+\dot{\mathbf{w}}_{i}(\mathbf{r}, \tau-t) \mathbf{u}_{i}(\mathbf{r}, t)\right) d t \\
= & \left.\sum_{i=1}^{3} \rho(\mathbf{r})\left(\dot{\mathbf{u}}_{i}(\mathbf{r}, t) \mathbf{w}_{i}(\mathbf{r}, \tau-t)+\dot{\mathbf{w}}_{i}(\mathbf{r}, \tau-t) \mathbf{u}_{i}(\mathbf{r}, t)\right)\right|_{t=\tau_{0}} ^{\tau-\tau_{0}} \\
= & \sum_{i=1}^{3} \rho(\mathbf{r})\left(\dot{\mathbf{u}}_{i}\left(\mathbf{r}, \tau-\tau_{0}\right) \mathbf{w}_{i}\left(\mathbf{r}, \tau_{0}\right)+\dot{\mathbf{w}}_{i}\left(\mathbf{r}, \tau_{0}\right) \mathbf{u}_{i}\left(\mathbf{r}, \tau-\tau_{0}\right)-\dot{\mathbf{u}}_{i}\left(\mathbf{r}, \tau_{0}\right) \mathbf{w}_{i}\left(\mathbf{r}, \tau-\tau_{0}\right)-\dot{\mathbf{w}}_{i}\left(\mathbf{r}, \tau-\tau_{0}\right) \mathbf{u}_{i}\left(\mathbf{r}, \tau_{0}\right)\right) \\
= & 0
\end{aligned}
$$

where in the last step we use the causality conditions that

$$
\begin{aligned}
\mathbf{u}_{i}\left(\mathbf{r}, \tau_{0}\right) & =\dot{\mathbf{u}}_{i}\left(\mathbf{r}, \tau_{0}\right)=0, \\
\mathbf{w}_{i}\left(\mathbf{r}, \tau_{0}\right) & =\dot{\mathbf{w}}_{i}\left(\mathbf{r}, \tau_{0}\right)=0 .
\end{aligned}
$$

Thus, we have in equation 64

$$
\int_{-\infty}^{\infty} d t \int_{V} \sum_{i=1}^{3} \rho(\mathbf{r})\left(\ddot{\mathbf{u}}_{i}(\mathbf{r}, t) \mathbf{w}_{i}(\mathbf{r}, \tau-t)-\ddot{\mathbf{w}}_{i}(\mathbf{r}, \tau-t) \mathbf{u}_{i}(\mathbf{r}, t)\right) d V=0,
$$

which proves the reciprocity theorem.

The reciprocity theorem is an important result as it gives the relationship between displacements produced by two different systems of forces. One important application of the theorem is the reciprocity of Green's tensor (see Section 8 for the definition of Green's tensor).

Lemma 12.3 (The Reciprocity of Green's Tensor) Under the homogeneous boundary conditions, the Green's tensor $\mathrm{G}$ has the following reciprocities:

$$
\begin{aligned}
\mathbf{G}_{m n}\left(\boldsymbol{\xi}_{2}, \tau ; \boldsymbol{\xi}_{1}, 0\right) & =\mathbf{G}_{n m}\left(\boldsymbol{\xi}_{1}, \tau ; \boldsymbol{\xi}_{2}, 0\right), \\
\mathbf{G}_{m n}\left(\boldsymbol{\xi}_{2}, \tau_{2} ; \boldsymbol{\xi}_{1}, \tau_{1}\right) & =\mathbf{G}_{n m}\left(\boldsymbol{\xi}_{1},-\tau_{1} ; \boldsymbol{\xi}_{2},-\tau_{2}\right),
\end{aligned}
$$

where $\boldsymbol{\xi}_{1}$ and $\boldsymbol{\xi}_{2}$ are two different position vectors; $\tau, \tau_{1}$ and $\tau_{2}$ are points of times.

Proof Assume that there are two volume force fields $\mathbf{f}$ and $\mathbf{g}$ formed by two different impulsive forces respectively:

$$
\begin{aligned}
& \mathbf{f}_{i}(\mathbf{r}, t)=A_{n} \delta\left(\mathbf{r}-\boldsymbol{\xi}_{1}\right) \delta\left(t-\tau_{1}\right) \delta_{i n} \quad n \in\{1,2,3\}, \\
& \mathbf{g}_{i}(\mathbf{r}, t)=A_{m} \delta\left(\mathbf{r}-\boldsymbol{\xi}_{2}\right) \delta\left(t+\tau_{2}\right) \delta_{i m} \quad m \in\{1,2,3\}
\end{aligned}
$$

for all $i \in\{1,2,3\}$ and all $\mathbf{r} \in V$. Their corresponding displacements $\mathbf{u}(\mathbf{r}, t)$ and $\mathbf{w}(\mathbf{r}, t)$ under the same boundary conditions can be expressed in terms of a same Green's tensor G:

$$
\begin{aligned}
\mathbf{u}_{i}(\mathbf{r}, t) & =A_{n} \mathbf{G}_{i n}\left(\mathbf{r}, t ; \boldsymbol{\xi}_{1}, \tau_{1}\right), \\
\mathbf{w}_{i}(\mathbf{r}, t) & =A_{m} \mathbf{G}_{i m}\left(\mathbf{r}, t ; \boldsymbol{\xi}_{2},-\tau_{2}\right) .
\end{aligned}
$$

Equation 67 and 69 can be rewritten after changing their input time variable $t$ :

$$
\begin{aligned}
\mathbf{g}_{i}(\mathbf{r}, \tau-t) & =A_{m} \delta\left(\mathbf{r}-\boldsymbol{\xi}_{2}\right) \delta\left(\tau-t+\tau_{2}\right) \delta_{i m} \\
\mathbf{w}_{i}(\mathbf{r}, \tau-t) & =A_{m} \mathbf{G}_{i m}\left(\mathbf{r}, \tau-t ; \boldsymbol{\xi}_{2},-\tau_{2}\right)
\end{aligned}
$$


Remind that the Reciprocity Theorem 12.2 gives:

$$
\begin{aligned}
& \int_{-\infty}^{\infty} d t \int_{V} \sum_{i=1}^{3}(\left.\mathbf{u}_{i}(\mathbf{r}, t) \mathbf{g}_{i}(\mathbf{r}, \tau-t)-\mathbf{w}_{i}(\mathbf{r}, \tau-t) \mathbf{f}_{i}(\mathbf{r}, t)\right) d V \\
& \quad=\int_{-\infty}^{\infty} d t \int_{S} \sum_{i=1}^{3}\left(\mathbf{w}_{i}(\mathbf{r}, \tau-t) \mathbf{t}_{i}(\mathbf{r}, \mathbf{n}(\mathbf{r}), t, \mathbf{u})-\mathbf{u}_{i}(\mathbf{r}, t) \mathbf{t}_{i}(\mathbf{r}, \mathbf{n}(\mathbf{r}), \tau-t, \mathbf{w})\right) d S
\end{aligned}
$$

Since both displacement fields have the same homogeneous boundary conditions satisfying

$$
\begin{aligned}
\mathbf{u}(\mathbf{r}, t)=\mathbf{w}(\mathbf{r}, t) & =\mathbf{0} \quad o r \\
\mathbf{t}(\mathbf{r}, \mathbf{n}(\mathbf{r}), t, \mathbf{u})=\mathbf{t}(\mathbf{r}, \mathbf{n}(\mathbf{r}), t, \mathbf{w}) & =\mathbf{0}
\end{aligned}
$$

at every $\mathbf{r}$ on surface $S$ throughout all time, we have the integral over $S$ in the reciprocity equation (72) vanished and obtain:

$$
\int_{-\infty}^{\infty} d t \int_{V} \sum_{i=1}^{3}\left(\mathbf{u}_{i}(\mathbf{r}, t) \mathbf{g}_{i}(\mathbf{r}, \tau-t)-\mathbf{w}_{i}(\mathbf{r}, \tau-t) \mathbf{f}_{i}(\mathbf{r}, t)\right) d V=0 .
$$

Plugging equations (66, 68, (70) and (71) into 273 gives:

$$
\begin{aligned}
\int_{-\infty}^{\infty} d t \int_{V} \sum_{i=1}^{3}\left(A_{n} \mathbf{G}_{i n}\left(\mathbf{r}, t ; \boldsymbol{\xi}_{1}, \tau_{1}\right)\right. & A_{m} \delta\left(\mathbf{r}-\boldsymbol{\xi}_{2}\right) \delta\left(\tau-t+\tau_{2}\right) \delta_{i m} \\
& \left.-A_{m} \mathbf{G}_{i m}\left(\mathbf{r}, \tau-t ; \boldsymbol{\xi}_{2},-\tau_{2}\right) A_{n} \delta\left(\mathbf{r}-\boldsymbol{\xi}_{1}\right) \delta\left(t-\tau_{1}\right) \delta_{i n}\right) d V=0 .
\end{aligned}
$$

Due to the definitions of Dirac delta function and Kronecker delta, we have equation (74) reduced to:

$$
\begin{gathered}
A_{n} A_{m} \mathbf{G}_{m n}\left(\boldsymbol{\xi}_{2}, \tau+\tau_{2} ; \boldsymbol{\xi}_{1}, \tau_{1}\right)-A_{n} A_{m} \mathbf{G}_{n m}\left(\boldsymbol{\xi}_{1}, \tau-\tau_{1} ; \boldsymbol{\xi}_{2},-\tau_{2}\right)=0 \\
\mathbf{G}_{m n}\left(\boldsymbol{\xi}_{2}, \tau+\tau_{2} ; \boldsymbol{\xi}_{1}, \tau_{1}\right)=\mathbf{G}_{n m}\left(\boldsymbol{\xi}_{1}, \tau-\tau_{1} ; \boldsymbol{\xi}_{2},-\tau_{2}\right) .
\end{gathered}
$$

Let $\tau_{1}=\tau_{2}=0$ in equation $(75)$, we then have

$$
\mathbf{G}_{m n}\left(\boldsymbol{\xi}_{2}, \tau ; \boldsymbol{\xi}_{1}, 0\right)=\mathbf{G}_{n m}\left(\boldsymbol{\xi}_{1}, \tau ; \boldsymbol{\xi}_{2}, 0\right),
$$

which proves the first relation that gives the spatial reciprocity of Green's tensor. Let $\tau=0$, equation (75) implies

$$
\mathbf{G}_{m n}\left(\boldsymbol{\xi}_{2}, \tau_{2} ; \boldsymbol{\xi}_{1}, \tau_{1}\right)=\mathbf{G}_{n m}\left(\boldsymbol{\xi}_{1},-\tau_{1} ; \boldsymbol{\xi}_{2},-\tau_{2}\right) \text {, }
$$

which proves the second relation that gives the spatial-temporal reciprocity of Green's tensor.

\subsection{Reciprocities in Elastostatics}

In the case of elastostatics, we can also obtain the following reciprocal relations:

- Betti's theorem for elastostatics: By neglecting time element $t$ and taking $\ddot{\mathbf{u}}_{i}(\mathbf{r}, t)$ and $\ddot{\mathbf{w}}_{i}(\mathbf{r}, t)$ to be zero in (54), we obtain the Betti's theorem for elastostatics:

$$
\begin{aligned}
\int_{V} \sum_{i=1}^{3} \mathbf{f}_{i}(\mathbf{r}) \mathbf{w}_{i}(\mathbf{r}) d V+\int_{S} \sum_{i=1}^{3} \mathbf{t}_{i}(\mathbf{r}, & \mathbf{n}(\mathbf{r}), \mathbf{u}) \mathbf{w}_{i}(\mathbf{r}) d S \\
& =\int_{V} \sum_{i=1}^{3} \mathbf{g}_{i}(\mathbf{r}) \mathbf{u}_{i}(\mathbf{r}) d V+\int_{S} \sum_{i=1}^{3} \mathbf{t}_{i}(\mathbf{r}, \mathbf{n}(\mathbf{r}), \mathbf{w}) \mathbf{u}_{i}(\mathbf{r}) d S ;
\end{aligned}
$$

- Reciprocity theorem for elastostatics: The reciprocity theorem for elastostatics is an immediate result by arranging (76):

$$
\int_{V} \sum_{i=1}^{3}\left(\mathbf{u}_{i}(\mathbf{r}) \mathbf{g}_{i}(\mathbf{r})-\mathbf{w}_{i}(\mathbf{r}) \mathbf{f}_{i}(\mathbf{r})\right) d V=\int_{S} \sum_{i=1}^{3}\left(\mathbf{w}_{i}(\mathbf{r}) \mathbf{t}_{i}(\mathbf{r}, \mathbf{n}(\mathbf{r}), \mathbf{u})-\mathbf{u}_{i}(\mathbf{r}) \mathbf{t}_{i}(\mathbf{r}, \mathbf{n}(\mathbf{r}), \mathbf{w})\right) d S
$$


- Reciprocity of Green's tensor of elastostatics: Let

$$
\begin{aligned}
\mathbf{f}_{i}(\mathbf{r}) & =A_{n} \delta\left(\mathbf{r}-\boldsymbol{\xi}_{1}\right) \delta_{i n}, \\
\mathbf{g}_{i}(\mathbf{r}) & =A_{m} \delta\left(\mathbf{r}-\boldsymbol{\xi}_{2}\right) \delta_{i m}, \\
\mathbf{u}_{i}(\mathbf{r}) & =A_{n} \mathbf{G}_{i n}\left(\mathbf{r} ; \boldsymbol{\xi}_{1}\right), \\
\mathbf{w}_{i}(\mathbf{r}) & =A_{m} \mathbf{G}_{i m}\left(\mathbf{r} ; \boldsymbol{\xi}_{2}\right)
\end{aligned}
$$

for $n \in\{1,2,3\}$ and $m \in\{1,2,3\}$. Then, under the homogeneous boundary conditions that

$$
\begin{aligned}
\mathbf{u}(\mathbf{r})=\mathbf{w}(\mathbf{r}) & =0 \quad \text { or } \\
\mathbf{t}(\mathbf{r}, \mathbf{n}(\mathbf{r}), \mathbf{u})=\mathbf{t}(\mathbf{r}, \mathbf{n}(\mathbf{r}), \mathbf{w})=0 &
\end{aligned}
$$

at every $\mathbf{r}$ on surface $S$, we can prove in a similar way to Lemma 12.3 that

$$
\mathbf{S}_{m n}\left(\boldsymbol{\xi}_{2} ; \boldsymbol{\xi}_{1}\right)=\mathbf{S}_{n m}\left(\boldsymbol{\xi}_{1} ; \boldsymbol{\xi}_{2}\right) \text {. }
$$

\section{Kelvin's Problem}

In 1848, Lord Kelvin (1848) gave the static displacement field due to a single point force in an infinite homogeneous isotropic elastic medium. The problem solved for the displacement field in this case is sometimes called the Kelvin's Problem and can be formulated mathematically as follow:

Problem 13.1 (Kelvin's problem) Find the displacement field

$$
\mathbf{u} \in \mathcal{E}(\lambda, \mu, \mathbf{f}, \Pi)
$$

with

such that

$$
\mathbf{f}_{i}(\mathbf{r})=\delta(\mathbf{r}-\boldsymbol{\xi}) \delta_{i n}
$$

a) $\int_{S} \sum_{j=1}^{3} \boldsymbol{\sigma}_{i j}(\mathbf{r}) \mathbf{n}_{j}(\mathbf{r}) d S+\int_{V} \mathbf{f}_{i}(\mathbf{r}) d V=0 \quad \forall V \subset \Pi$;

b) $\mathbf{u}_{i}(\mathbf{r})=\mathcal{O}\left(d^{-1}\right), \boldsymbol{\sigma}_{i j}(\mathbf{r})=\mathcal{O}\left(d^{-2}\right)$ for all $i, j \in\{1,2,3\}$ as $d \rightarrow \infty$;

c) $\mathbf{u}_{i}(\mathbf{r})=\mathcal{O}\left(d^{-1}\right), \boldsymbol{\sigma}_{i j}(\mathbf{r})=\mathcal{O}\left(d^{-2}\right)$ for all $i, j \in\{1,2,3\}$ as $d \rightarrow 0$,

where $\lambda$ and $\mu$ in (78) are Lamé coefficients; $\Pi$ represents the infinite medium; expression (79) shows that the volume force field is formed by an impulsive force acting at $\boldsymbol{\xi}$ along the direction of unit vector $\mathbf{e}^{n}$; condition $\boldsymbol{a}$ ) is the elastostatic equation of motion that displacement field $\mathbf{u}$ needs to fulfil; $S$ and $V$ denote the surface and volume of any region in the infinite medium that encloses position $\boldsymbol{\xi}$; condition $\boldsymbol{b}$ ) gives the boundary conditions of the problem requiring the displacements and stresses to vanish at infinity; condition c) requires displacement and stresses to be unbounded at the force-acting position; $d=\|\mathbf{r}-\boldsymbol{\xi}\|$ is the Euclidean distance between the displacement-evaluating position $\mathbf{r}$ and the force-acting position $\boldsymbol{\xi}$.

The conditions a), b) and c) are sufficient to uniquely determine the solutions of the Kelvin's problem (Sternberg 1980). We present the solution of the Kelvin's problem [13.1] in the form of the Green's tensor of elastostatics defined in Section 8

$$
\mathbf{S}_{i n}(\mathbf{r} ; \boldsymbol{\xi})=\frac{1}{8 \pi \mu}\left((2-\nu) \frac{\delta_{i n}}{d}+\nu \frac{\left(\mathbf{r}_{i}-\boldsymbol{\xi}_{i}\right)\left(\mathbf{r}_{n}-\boldsymbol{\xi}_{n}\right)}{d^{3}}\right)
$$

for all $i, n \in\{1,2,3\}$, where

- $\nu=\frac{\lambda+\mu}{\lambda+2 \mu}$;

- $d=\|\mathbf{r}-\boldsymbol{\xi}\|=\sqrt{\sum_{k=1}^{3}\left(\mathbf{r}_{k}-\boldsymbol{\xi}_{k}\right)^{2}}$.

The Green's tensor [80] to the Kelvin's problem is called the Somigliana's tensor and its derivation can be found in Lay \& Wallace (1995) and Bower (2005). 


\section{Mindlin's Problem}

The solution of the Kelvin's problem is a fundamental result for linear elastic theory. However, in seismology, we are more interested in displacement fields due to a single point force exerted in a half space as this mimics the Earth and the boundary of the half space can be treated as the surface of the Earth. For this reason, in this section we present the displacement solution for a semi-infinite homogeneous isotropic medium in elastostatic case. The problem on finding such a solution is sometimes called the Mindlin's Problem as it is solved by Mindlin (1936) in 1936 by the method of images and derived again by Mindlin (1953) in 1953 using potential theory. The following is the mathematical formulation of the problem.

Problem 14.1 (Mindlin's problem) Find the displacement field

$$
\mathbf{u} \in \mathcal{E}(\lambda, \mu, \mathbf{f}, P \times \tilde{\Pi})
$$

with

such that

$$
\mathbf{f}_{i}(\mathbf{r})=\delta(\mathbf{r}-\boldsymbol{\xi}) \delta_{i n}
$$

a) $\int_{S} \sum_{j=1}^{3} \boldsymbol{\sigma}_{i j}(\mathbf{r}) \mathbf{n}_{j}(\mathbf{r}) d S+\int_{V} \mathbf{f}_{i}(\mathbf{r}) d V=0 \quad \forall V \subset \tilde{\Pi}$;

b) $\boldsymbol{\sigma}(\mathbf{r}) \mathbf{e}^{3}=\mathbf{0} \quad \forall \mathbf{r} \in P$;

c) $\mathbf{u}_{i}(\mathbf{r})=\mathcal{O}\left(d^{-1}\right), \boldsymbol{\sigma}_{i j}(\mathbf{r})=\mathcal{O}\left(d^{-2}\right)$ for all $i, j \in\{1,2,3\}$ as $d \rightarrow \infty$;

d) $\mathbf{u}_{i}(\mathbf{r})=\mathcal{O}\left(d^{-1}\right), \boldsymbol{\sigma}_{i j}(\mathbf{r})=\mathcal{O}\left(d^{-2}\right)$ for all $i, j \in\{1,2,3\}$ as $d \rightarrow 0$,

where $\lambda$ and $\mu$ in (81) are Lamé coefficients; $\tilde{\Pi}$ represents the semi-infinite medium and P(xy-plane) represents the boundary of the medium; expression (82) shows that the volume force field is formed by an impulsive force acting at $\boldsymbol{\xi}$ along the direction of unit vector $\mathbf{e}^{n}$; condition $\left.\mathbf{a}\right)$ is the elastostatic equation of motion that displacement field $\mathbf{u}$ needs to fulfil; $S$ and $V$ denote the surface and volume of any region in the semi-infinite medium that encloses position $\boldsymbol{\xi}$; condition $\boldsymbol{b})$ and $\boldsymbol{c}$ ) give the boundary conditions of the problem requiring the surface $P$ to be stress-free and the displacements and stresses to vanish at infinity; condition d) requires displacement and stresses to be unbounded at the force-acting position; $d=\|\mathbf{r}-\boldsymbol{\xi}\|$ is the Euclidean distance between the displacement-evaluating position $\mathbf{r}$ and the force-acting position $\boldsymbol{\xi}$.

The conditions a), b), c) and d) ensures the uniqueness of the solution of the Mindlin's problem. In Mindlin \& Cheng (1950) the displacement field is given by assuming that the single point force is exerted on the $z$-axis beneath the free surface $P$, while in Press (1965) the displacement field is given with the single point force acted on $x z$-plane beneath the free surface $P$. More generally, Kachanov et al. (2013) presents the displacement field with the single point force exerted at an arbitrary position in the semi-infinite medium. In this note, we adopt the form used by Okada (1992):

$$
\mathbf{S}_{i n}(\mathbf{r} ; \boldsymbol{\xi})=f_{1}\left(i, n, \mathbf{r}^{\prime}, \boldsymbol{\xi}\right)-f_{1}(i, n, \mathbf{r}, \boldsymbol{\xi})+f_{2}(i, n, \mathbf{r}, \boldsymbol{\xi})+\mathbf{r}_{3} f_{3}(i, n, \mathbf{r}, \boldsymbol{\xi}),
$$

for all $i, n \in\{1,2,3\}$, where

$$
\begin{aligned}
\bullet f_{1}(i, n, \mathbf{r}, \boldsymbol{\xi})= & \frac{1}{8 \pi \mu}\left((2-\nu) \frac{\delta_{i n}}{R(\mathbf{r}, \boldsymbol{\xi})}+\nu \frac{R_{i}(\mathbf{r}, \boldsymbol{\xi}) R_{n}(\mathbf{r}, \boldsymbol{\xi})}{R^{3}(\mathbf{r}, \boldsymbol{\xi})}\right) \\
\bullet f_{2}(i, n, \mathbf{r}, \boldsymbol{\xi})= & \frac{1}{4 \pi \mu}\left(\frac{\delta_{i n}}{R(\mathbf{r}, \boldsymbol{\xi})}+\frac{R_{i}(\mathbf{r}, \boldsymbol{\xi}) R_{n}(\mathbf{r}, \boldsymbol{\xi})}{R^{3}(\mathbf{r}, \boldsymbol{\xi})}+\frac{1-\nu}{\nu}\left[\frac{\delta_{i n}}{R(\mathbf{r}, \boldsymbol{\xi})+R_{3}(\mathbf{r}, \boldsymbol{\xi})}\right.\right. \\
& +\frac{R_{i}(\mathbf{r}, \boldsymbol{\xi}) \delta_{n 3}-R_{n}(\mathbf{r}, \boldsymbol{\xi}) \delta_{i 3}\left(1-\delta_{n 3}\right)}{R(\mathbf{r}, \boldsymbol{\xi})\left(R(\mathbf{r}, \boldsymbol{\xi})+R_{3}(\mathbf{r}, \boldsymbol{\xi})\right)} \\
& \left.\left.-\frac{R_{i}(\mathbf{r}, \boldsymbol{\xi}) R_{n}(\mathbf{r}, \boldsymbol{\xi})}{R(\mathbf{r}, \boldsymbol{\xi})\left(R(\mathbf{r}, \boldsymbol{\xi})+R_{3}(\mathbf{r}, \boldsymbol{\xi})\right)^{2}}\left(1-\delta_{i 3}\right)\left(1-\delta_{n 3}\right)\right]\right) ; \\
\bullet f_{3}(i, n, \mathbf{r}, \boldsymbol{\xi})= & \frac{1}{4 \pi \mu}\left(1-2 \delta_{i 3}\right)\left((2-\nu) \frac{R_{i}(\mathbf{r}, \boldsymbol{\xi}) \delta_{n 3}-R_{n}(\mathbf{r}, \boldsymbol{\xi}) \delta_{i 3}}{R^{3}(\mathbf{r}, \boldsymbol{\xi})}+\nu \boldsymbol{\xi}_{3}\left[\frac{\delta_{i n}}{R^{3}(\mathbf{r}, \boldsymbol{\xi})}-\frac{3 R_{i}(\mathbf{r}, \boldsymbol{\xi}) R_{n}(\mathbf{r}, \boldsymbol{\xi})}{R^{5}(\mathbf{r}, \boldsymbol{\xi})}\right]\right)
\end{aligned}
$$


- $\mathbf{r}^{\prime}=\left[\begin{array}{ccc}1 & 0 & 0 \\ 0 & 1 & 0 \\ 0 & 0 & -1\end{array}\right] \mathbf{r}$ is the reflection of $\mathbf{r}$ with respect to the free surface;

- $\nu=\frac{\lambda+\mu}{\lambda+2 \mu}$;

- $R_{1}(\mathbf{r}, \boldsymbol{\xi})=\mathbf{r}_{1}-\boldsymbol{\xi}_{1}, R_{2}(\mathbf{r}, \boldsymbol{\xi})=\mathbf{r}_{2}-\boldsymbol{\xi}_{2}$ and $R_{3}(\mathbf{r}, \boldsymbol{\xi})=-\mathbf{r}_{3}-\boldsymbol{\xi}_{3}$;

- $R(\mathbf{r}, \boldsymbol{\xi})=\sqrt{{R_{1}}^{2}(\mathbf{r}, \boldsymbol{\xi})+{R_{2}}^{2}(\mathbf{r}, \boldsymbol{\xi})+{R_{3}}^{2}(\mathbf{r}, \boldsymbol{\xi})}$.

Note that the first term $f_{1}\left(i, n, \mathbf{r}^{\prime}, \boldsymbol{\xi}\right)$ in $(83)$ is the Somigliana's tensor, representing the displacement contributed by a single point force exerted at $\boldsymbol{\xi}$ in an infinite medium; the second term $-f_{1}(i, n, \mathbf{r}, \boldsymbol{\xi})$ in $(83)$ represents the displacement changes due to an image single point force acted at $\left(\boldsymbol{\xi}_{1}, \boldsymbol{\xi}_{2},-\boldsymbol{\xi}_{3}\right)$ in an infinite medium; $f_{2}(i, n, \mathbf{r}, \boldsymbol{\xi})$ in 83 is a surface deformation related term as when $\mathbf{r}_{3}=0$ the term represents the surface displacement due to a single point force in a half space; the last term $f_{3}(i, n, \mathbf{r}, \boldsymbol{\xi})$ in 83 is simply a multiplier of the depth of the observation point $\mathbf{r}$.

The proof for the solution to the Mindlin's problem has been illustrated by Chau (2012) using potential theory.

\section{Representation Theorem}

Theorem 15.1 (Representation theorem) Assume there is a continuous elastic medium with volume $V$ and surface $S$. Suppose that

$$
\begin{array}{r}
\mathbf{u} \in \mathcal{E}(\varrho, \mathbf{c}, \mathbf{f}, V \times S \times[-\infty, \infty]), \\
\mathbf{w} \in \mathcal{E}(\varrho, \mathbf{c}, \mathbf{g}, V \times S \times[-\infty, \infty])
\end{array}
$$

are two displacement fields caused respectively by $\mathbf{f}$ and $\mathbf{g}$ under generally different initial and boundary conditions. Then, if the volume force field $\mathbf{g}$ is formed by an impulsive force, i.e.,

$$
\mathbf{g}_{i}(\mathbf{r}, t)=A_{n} \delta(\mathbf{r}-\boldsymbol{\xi}) \delta(t) \delta_{i n} \quad n \in\{1,2,3\}
$$

for all $i \in\{1,2,3\}$ and all $\mathbf{r} \in V$, with its corresponding displacement field

$$
\mathbf{w}_{i}(\mathbf{r}, t)=A_{n} \mathbf{G}_{i n}^{\mathbf{w}}(\mathbf{r}, t ; \boldsymbol{\xi}, 0),
$$

the following representation of the displacement field $\mathbf{u}$ can be obtained under the causality principle:

$$
\begin{aligned}
\mathbf{u}_{n}(\mathbf{r}, t)=\int_{-\infty}^{\infty} d \tau \int_{V} \sum_{i=1}^{3} \mathbf{G}_{i n}^{\mathbf{w}}(\boldsymbol{\xi}, t-\tau ; \mathbf{r}, 0) \mathbf{f}_{i}(\boldsymbol{\xi}, \tau) d V \\
+\int_{-\infty}^{\infty} d \tau \int_{S} \sum_{i=1}^{3}\left(\mathbf{G}_{i n}^{\mathbf{w}}(\boldsymbol{\xi}, t-\tau ; \mathbf{r}, 0) \mathbf{t}_{i}(\boldsymbol{\xi}, \mathbf{n}(\boldsymbol{\xi}), \tau, \mathbf{u})\right. \\
\left.\quad-\mathbf{u}_{i}(\boldsymbol{\xi}, \tau) \sum_{j=1}^{3} \sum_{k=1}^{3} \sum_{l=1}^{3} \mathbf{c}_{i j k l}(\boldsymbol{\xi}) \frac{\partial \mathbf{G}_{k n}^{\mathbf{w}}(\boldsymbol{\xi}, t-\tau ; \mathbf{r}, 0)}{\partial \boldsymbol{\xi}_{l}} \mathbf{n}_{j}(\boldsymbol{\xi})\right) d S .
\end{aligned}
$$

Proof Remind that the Reciprocity Theorem 12.2 states that

$$
\begin{aligned}
\int_{-\infty}^{\infty} d t \int_{V} \sum_{i=1}^{3}\left(\mathbf{u}_{i}(\mathbf{r}, t) \mathbf{g}_{i}(\mathbf{r}, \tau-t)-\mathbf{w}_{i}(\mathbf{r}, \tau-t) \mathbf{f}_{i}(\mathbf{r}, t)\right) d V \\
=\int_{-\infty}^{\infty} d t \int_{S} \sum_{i=1}^{3}\left(\mathbf{w}_{i}(\mathbf{r}, \tau-t) \mathbf{t}_{i}(\mathbf{r}, \mathbf{n}(\mathbf{r}), t, \mathbf{u})-\mathbf{u}_{i}(\mathbf{r}, t) \mathbf{t}_{i}(\mathbf{r}, \mathbf{n}(\mathbf{r}), \tau-t, \mathbf{w})\right) d S
\end{aligned}
$$


for some $\tau \in \mathbb{R}$. According to Gauchy's Stress Theorem 4, Lemma 6.2 and expression (85), we have

$$
\begin{aligned}
\mathbf{t}_{i}(\mathbf{r}, \mathbf{n}(\mathbf{r}), \tau-t, \mathbf{w}) & =\sum_{j=1}^{3} \boldsymbol{\sigma}_{i j}(\mathbf{r}, \tau-t, \mathbf{w}) \mathbf{n}_{j}(\mathbf{r}) \\
& =\sum_{j=1}^{3} \sum_{k=1}^{3} \sum_{l=1}^{3} \mathbf{c}_{i j k l}(\mathbf{r}) \frac{\partial \mathbf{w}_{k}(\mathbf{r}, \tau-t)}{\partial \mathbf{r}_{l}} \mathbf{n}_{j}(\mathbf{r}) \\
& =A_{n} \sum_{j=1}^{3} \sum_{k=1}^{3} \sum_{l=1}^{3} \mathbf{c}_{i j k l}(\mathbf{r}) \frac{\partial \mathbf{G}_{k n}^{\mathbf{w}}(\mathbf{r}, \tau-t ; \boldsymbol{\xi}, 0)}{\partial \mathbf{r}_{l}} \mathbf{n}_{j}(\mathbf{r})
\end{aligned}
$$

Inserting expressions (84), (85) and 87 into equation 86 gives

$$
\begin{aligned}
& \int_{-\infty}^{\infty} d t \int_{V} \sum_{i=1}^{3}\left(\mathbf{u}_{i}(\mathbf{r}, t)\right.\left.A_{n} \delta(\mathbf{r}-\boldsymbol{\xi}) \delta(\tau-t) \delta_{i n}-A_{n} \mathbf{G}_{i n}^{\mathbf{w}}(\mathbf{r}, \tau-t ; \boldsymbol{\xi}, 0) \mathbf{f}_{i}(\mathbf{r}, t)\right) d V \\
&=\int_{-\infty}^{\infty} d t \int_{S} \sum_{i=1}^{3}\left(A_{n} \mathbf{G}_{i n}^{\mathbf{w}}(\mathbf{r}, \tau-t ; \boldsymbol{\xi}, 0) \mathbf{t}_{i}(\mathbf{r}, \mathbf{n}(\mathbf{r}), t, \mathbf{u})\right. \\
&\left.\quad-\mathbf{u}_{i}(\mathbf{r}, t) A_{n} \sum_{j=1}^{3} \sum_{k=1}^{3} \sum_{l=1}^{3} \mathbf{c}_{i j k l}(\mathbf{r}) \frac{\partial \mathbf{G}_{k n}^{\mathbf{w}}(\mathbf{r}, \tau-t ; \boldsymbol{\xi}, 0)}{\partial \mathbf{r}_{l}} \mathbf{n}_{j}(\mathbf{r})\right) d S
\end{aligned}
$$

Note that

$$
\int_{-\infty}^{\infty} d t \int_{V} \sum_{i=1}^{3} \mathbf{u}_{i}(\mathbf{r}, t) A_{n} \delta(\mathbf{r}-\boldsymbol{\xi}) \delta(\tau-t) \delta_{i n} d V=A_{n} \mathbf{u}_{n}(\boldsymbol{\xi}, \tau) .
$$

Thus, equation 88 becomes

$$
\begin{aligned}
& \mathbf{u}_{n}(\boldsymbol{\xi}, \tau)=\int_{-\infty}^{\infty} d t \int_{V} \sum_{i=1}^{3} \mathbf{G}_{i n}^{\mathbf{w}}(\mathbf{r}, \tau-t ; \boldsymbol{\xi}, 0) \mathbf{f}_{i}(\mathbf{r}, t) d V \\
&+\int_{-\infty}^{\infty} d t \int_{S} \sum_{i=1}^{3}\left(\mathbf{G}_{i n}^{\mathbf{w}}(\mathbf{r}, \tau-t ; \boldsymbol{\xi}, 0) \mathbf{t}_{i}(\mathbf{r}, \mathbf{n}(\mathbf{r}), t, \mathbf{u})\right. \\
&\left.\quad-\mathbf{u}_{i}(\mathbf{r}, t) \sum_{j=1}^{3} \sum_{k=1}^{3} \sum_{l=1}^{3} \mathbf{c}_{i j k l}(\mathbf{r}) \frac{\partial \mathbf{G}_{k n}^{\mathbf{w}}(\mathbf{r}, \tau-t ; \boldsymbol{\xi}, 0)}{\partial \mathbf{r}_{l}} \mathbf{n}_{j}(\mathbf{r})\right) d S
\end{aligned}
$$

For the notational convenience, we would like to make the displacement to be evaluated at position $\mathbf{r}$ and time $t$. Therefore, by interchanging $\boldsymbol{\xi}$ with $\mathbf{r}$ and $\tau$ with $t$ in equation 89 ) and noting that the symbol-interchange does not affect the equality of (89), we have

$$
\begin{aligned}
& \mathbf{u}_{n}(\mathbf{r}, t)=\int_{-\infty}^{\infty} d \tau \int_{V} \sum_{i=1}^{3} \mathbf{G}_{i n}^{\mathbf{w}}(\boldsymbol{\xi}, t-\tau ; \mathbf{r}, 0) \mathbf{f}_{i}(\boldsymbol{\xi}, \tau) d V \\
& \quad+\int_{-\infty}^{\infty} d \tau \int_{S} \sum_{i=1}^{3}\left(\mathbf{G}_{i n}^{\mathbf{w}}(\boldsymbol{\xi}, t-\tau ; \mathbf{r}, 0) \mathbf{t}_{i}(\boldsymbol{\xi}, \mathbf{n}(\boldsymbol{\xi}), \tau, \mathbf{u})\right. \\
&\left.\quad-\mathbf{u}_{i}(\boldsymbol{\xi}, \tau) \sum_{j=1}^{3} \sum_{k=1}^{3} \sum_{l=1}^{3} \mathbf{c}_{i j k l}(\boldsymbol{\xi}) \frac{\partial \mathbf{G}_{k n}^{\mathbf{w}}(\boldsymbol{\xi}, t-\tau ; \mathbf{r}, 0)}{\partial \boldsymbol{\xi}_{l}} \mathbf{n}_{j}(\boldsymbol{\xi})\right) d S,
\end{aligned}
$$

which proves the theorem.

Remark From this section onwards, we will use notation $\mathbf{G}^{\mathbf{w}}$ to represent the Green's tensor corresponding to the displacement field $\mathbf{w}$. Since different boundary conditions will lead to different displacement fields and thus different Green's tensors, it is necessary to make different notations to differentiate Green's tensors subject to distinct boundary conditions. 
It should be noted that after symbol-interchanges from 89 , to 90 , the integrals over volume $V$ and surface $S$ are evaluated with respect to $\boldsymbol{\xi}$ instead of $\mathbf{r}$. The Representation Theorem 15.1 states that the displacement at a certain position is contributed by the volume force field, the tractions (i.e., stresses on surface) as well as displacements on surface. However, the weights of these three contributions are not satisfactory as the Green's tensor components they involve indicate that the volume force field $\mathbf{g}$ is formed by an impulsive force placed at $\mathbf{r}$ and the observation point is at $\boldsymbol{\xi}$. We would like to have the force to be acted at $\boldsymbol{\xi}$ and the observation at $\mathbf{r}$ so that $\mathbf{u}(\mathbf{r}, t)$ can be treated as the sum of displacements on the surface and those caused by tractions and volume forces. To achieve this, we need the reciprocity of Green's tensor in Lemma 12.3 The application of the lemma, however, requires homogeneous boundary conditions, which state that at every position on the surface either the displacement or the traction is zero. In the rest of this section, we present two forms of the representation theorem under two special cases of the homogeneous boundary conditions.

- Rigid boundary: A rigid boundary on $\mathbf{w}$ (or $\mathbf{G}^{\mathbf{w}}$ ) indicates that the displacement field w (or the Green's tensor $\mathbf{G}^{\mathbf{w}}$ ) is constrained by the boundary condition that displacement at each position on the surface $S$ is zero. Mathematically, we have

$$
\mathbf{G}^{\mathbf{w}}(\boldsymbol{\xi}, t-\tau ; \mathbf{r}, 0)=\mathbf{0} \quad \forall \boldsymbol{\xi} \in S .
$$

Plugging (91) into 90, and invoking Lemma (12.3) then give the representation theorem under a rigid boundary:

$$
\begin{aligned}
\mathbf{u}_{n}(\mathbf{r}, t)=\int_{-\infty}^{\infty} & d \tau \int_{V} \sum_{i=1}^{3} \mathbf{G}_{n i}^{\mathbf{w}}(\mathbf{r}, t-\tau ; \boldsymbol{\xi}, 0) \mathbf{f}_{i}(\boldsymbol{\xi}, \tau) d V \\
& -\int_{-\infty}^{\infty} d \tau \int_{S} \sum_{i=1}^{3} \mathbf{u}_{i}(\boldsymbol{\xi}, \tau) \sum_{j=1}^{3} \sum_{k=1}^{3} \sum_{l=1}^{3} \mathbf{c}_{i j k l}(\boldsymbol{\xi}) \frac{\partial \mathbf{G}_{n k}^{\mathbf{w}}(\mathbf{r}, t-\tau ; \boldsymbol{\xi}, 0)}{\partial \boldsymbol{\xi}_{l}} \mathbf{n}_{j}(\boldsymbol{\xi}) d S
\end{aligned}
$$

- Traction-free boundary: A traction-free boundary gives the boundary condition that the tractions (i.e., stresses on the surface $S$ ) due to $\mathbf{w}$ (or $\mathbf{G}^{\mathbf{w}}$ ) are zero. Thus, we have

$$
\mathbf{t}_{i}(\boldsymbol{\xi}, \mathbf{n}(\boldsymbol{\xi}), \tau-t, \mathbf{w})=A_{n} \sum_{j=1}^{3} \sum_{k=1}^{3} \sum_{l=1}^{3} \mathbf{c}_{i j k l}(\boldsymbol{\xi}) \frac{\partial \mathbf{G}_{k n}^{\mathbf{w}}(\boldsymbol{\xi}, t-\tau ; \mathbf{r}, 0)}{\partial \boldsymbol{\xi}_{l}} \mathbf{n}_{j}(\boldsymbol{\xi})=0
$$

for all $\boldsymbol{\xi} \in S$ and all $i \in\{1,2,3\}$. Inserting (93) into (90) and invoking Lemma (12.3) then give the representation theorem under a traction-free boundary:

$$
\begin{aligned}
\mathbf{u}_{n}(\mathbf{r}, t)=\int_{-\infty}^{\infty} d \tau \int_{V} \sum_{i=1}^{3} \mathbf{G}_{n i}^{\mathbf{w}}(\mathbf{r}, t & -\tau ; \boldsymbol{\xi}, 0) \mathbf{f}_{i}(\boldsymbol{\xi}, \tau) d V \\
& +\int_{-\infty}^{\infty} d \tau \int_{S} \sum_{i=1}^{3} \mathbf{G}_{n i}^{\mathbf{w}}(\mathbf{r}, t-\tau ; \boldsymbol{\xi}, 0) \mathbf{t}_{i}(\boldsymbol{\xi}, \mathbf{n}(\boldsymbol{\xi}), \tau, \mathbf{u}) d S
\end{aligned}
$$

Note that the Green's tensors $\mathbf{G}^{\mathbf{w}}$ in 92 and $(94)$ are different as they are under different boundary conditions.

\subsection{Representation Theorem in Elastostatics}

The deviation of the representation theorem in elastostatics can be done in a similar fashion to that of the Representation Theorem 15.1 in elastodynamics. From the Reciprocity Theorem (77) for elastostatics, we obtain the elastostatic representation theorem:

$$
\begin{array}{r}
\mathbf{u}_{n}(\mathbf{r})=\int_{V} \sum_{i=1}^{3} \mathbf{S}_{i n}^{\mathbf{w}}(\boldsymbol{\xi} ; \mathbf{r}) \mathbf{f}_{i}(\boldsymbol{\xi}) d V+\int_{S} \sum_{i=1}^{3}\left(\mathbf{S}_{i n}^{\mathbf{w}}(\boldsymbol{\xi} ; \mathbf{r}) \mathbf{t}_{i}(\boldsymbol{\xi}, \mathbf{n}(\boldsymbol{\xi}), \mathbf{u})\right. \\
\left.-\mathbf{u}_{i}(\boldsymbol{\xi}) \sum_{j=1}^{3} \sum_{k=1}^{3} \sum_{l=1}^{3} \mathbf{c}_{i j k l}(\boldsymbol{\xi}) \frac{\partial \mathbf{S}_{k n}^{\mathbf{w}}(\boldsymbol{\xi} ; \mathbf{r})}{\partial \boldsymbol{\xi}_{l}} \mathbf{n}_{j}(\boldsymbol{\xi})\right) d S .
\end{array}
$$




\section{Representation Theorem with an Internal Surface}

In the last section, we presented the representation theorem in an elastic medium with volume $V$ and surface $S$. In this section, we give out the representation theorem with an internal surface $\varsigma$, which has two adjacent surfaces $\varsigma^{+}$and $\varsigma^{-}$ representing the opposite faces of a fault plane.

When an earthquake happens, dislocation (slip) will occur across $\varsigma$, causing discontinuities on displacements across $\varsigma$. In another word, the displacements on $\varsigma^{-}$may be different from those on $\varsigma^{+}$. To derive the representation theorem in this section, we first denote the displacement discontinuity (or dislocation) at position $\boldsymbol{\eta} \in \varsigma$ at time $t$ by

$$
\Delta \mathbf{u}(\boldsymbol{\eta}, t)=\left.\mathbf{u}(\boldsymbol{\eta}, t)\right|_{\varsigma^{+}}-\left.\mathbf{u}(\boldsymbol{\eta}, t)\right|_{\varsigma^{-}},
$$

where $\left.\mathbf{u}(\boldsymbol{\eta}, t)\right|_{\varsigma^{+}}$and $\left.\mathbf{u}(\boldsymbol{\eta}, t)\right|_{\varsigma^{-}}$are respectively the displacement at position $\boldsymbol{\eta}$ on side $\varsigma^{+}$and $\varsigma^{-}$at time $t$. In addition, we denote the unit normal vector to the surface element $d \varsigma^{-}$at position $\boldsymbol{\eta}$ as $\mathbf{v}(\boldsymbol{\eta})$, which points from $\varsigma^{-}$to $\varsigma^{+}$. Since $\varsigma^{-}$and $\varsigma^{+}$are opposite, the normal vector to the surface element $d \varsigma^{+}$at position $\boldsymbol{\eta}$ is simply $-\mathbf{v}(\boldsymbol{\eta})$, which points from $\varsigma^{+}$to $\varsigma^{-}$. Although $\varsigma^{+}$and $\varsigma^{-}$have different orientations (specified by opposite unit normal vectors), they contain the same set of positions as $\varsigma$ :

$$
\varsigma=\varsigma^{+}=\varsigma^{-}
$$

As there are displacement discontinuities in the medium, the equation of motion is invalid in the interior of $S$ (see Section 7 for the assumptions on the equation of motion). However, it is valid in the interior of $S+\varsigma^{+}+\varsigma^{-}$.

Remark Despite of the discontinuities of displacements on $\varsigma$, there can be traction discontinuities, which are defined by

$$
\left.\mathbf{t}(\boldsymbol{\eta},-\mathbf{v}(\boldsymbol{\eta}), t, \mathbf{u})\right|_{\varsigma^{+}}+\left.\mathbf{t}(\boldsymbol{\eta}, \mathbf{v}(\boldsymbol{\eta}), t, \mathbf{u})\right|_{\varsigma^{-}}
$$

for all $\boldsymbol{\eta} \in \varsigma$ at time $t$.

Theorem 16.1 (Representation theorem with an internal surface) Assume that there is a elastic medium with volume $V$ and surface $S$ and consists of an internal surface $\varsigma$ with opposite faces $\varsigma^{-}$and $\varsigma^{+}$. Then, if displacement fields $\mathbf{u}$ and $\mathbf{w}$ satisfy the same homogeneous boundary conditions on $S$ and the Green's tensor $\mathbf{G}^{\mathbf{w}}$ satisfies equation of motion on $\varsigma$, the representation for $\mathbf{u}$ is given by

$$
\begin{aligned}
\mathbf{u}_{n}(\mathbf{r}, t)= & \int_{-\infty}^{\infty} d \tau \int_{V} \sum_{i=1}^{3} \mathbf{G}_{n i}^{\mathbf{w}}(\mathbf{r}, t-\tau ; \boldsymbol{\xi}, 0) \mathbf{f}_{i}(\boldsymbol{\xi}, \tau) d V \\
& +\int_{-\infty}^{\infty} d \tau\left\{\int_{\varsigma} \sum_{i=1}^{3} \mathbf{G}_{n i}^{\mathbf{w}}(\mathbf{r}, t-\tau ; \boldsymbol{\eta}, 0)\left(\left.\mathbf{t}_{i}(\boldsymbol{\eta},-\mathbf{v}(\boldsymbol{\eta}), \tau, \mathbf{u})\right|_{\varsigma^{+}}+\left.\mathbf{t}_{i}(\boldsymbol{\eta}, \mathbf{v}(\boldsymbol{\eta}), \tau, \mathbf{u})\right|_{\varsigma^{-}}\right) d \varsigma\right. \\
& \left.+\int_{\varsigma} \sum_{i=1}^{3} \Delta \mathbf{u}_{i}(\boldsymbol{\eta}, \tau) \sum_{j=1}^{3} \sum_{k=1}^{3} \sum_{l=1}^{3} \mathbf{c}_{i j k l}(\boldsymbol{\eta}) \frac{\partial \mathbf{G}_{n k}^{\mathbf{w}}(\mathbf{r}, t-\tau ; \boldsymbol{\eta}, 0)}{\partial \boldsymbol{\eta}_{l}} \mathbf{v}_{j}(\boldsymbol{\eta}) d \varsigma\right\}
\end{aligned}
$$


Proof Since the equation of motion is valid in the interior of $S+\varsigma^{+}+\varsigma^{+}$, we have from the Representation Theorem 15.1 that

$$
\begin{aligned}
& \mathbf{u}_{n}(\mathbf{r}, t)=\int_{-\infty}^{\infty} d \tau \int_{V} \sum_{i=1}^{3} \mathbf{G}_{i n}^{\mathbf{w}}(\boldsymbol{\xi}, t-\tau ; \mathbf{r}, 0) \mathbf{f}_{i}(\boldsymbol{\xi}, \tau) d V \\
& +\int_{-\infty}^{\infty} d \tau\left\{\int_{\varsigma^{+}} \sum_{i=1}^{3} \mathbf{G}_{i n}^{\mathbf{w}}(\boldsymbol{\eta}, t-\tau ; \mathbf{r}, 0) \mathbf{t}_{i}(\boldsymbol{\eta},-\mathbf{v}(\boldsymbol{\eta}), \tau, \mathbf{u}) d \varsigma^{+}\right. \\
& +\int_{\varsigma^{-}} \sum_{i=1}^{3} \mathbf{G}_{i n}^{\mathbf{w}}(\boldsymbol{\eta}, t-\tau ; \mathbf{r}, 0) \mathbf{t}_{i}(\boldsymbol{\eta}, \mathbf{v}(\boldsymbol{\eta}), \tau, \mathbf{u}) d \varsigma^{-} \\
& -\int_{\varsigma^{+}} \sum_{i=1}^{3} \mathbf{u}_{i}(\boldsymbol{\eta}, \tau) \sum_{j=1}^{3} \sum_{k=1}^{3} \sum_{l=1}^{3} \mathbf{c}_{i j k l}(\boldsymbol{\eta}) \frac{\partial \mathbf{G}_{k n}^{\mathbf{w}}(\boldsymbol{\eta}, t-\tau ; \mathbf{r}, 0)}{\partial \boldsymbol{\eta}_{l}}\left(-\mathbf{v}_{j}(\boldsymbol{\eta})\right) d \varsigma^{+} \\
& \left.-\int_{\varsigma^{-}} \sum_{i=1}^{3} \mathbf{u}_{i}(\boldsymbol{\eta}, \tau) \sum_{j=1}^{3} \sum_{k=1}^{3} \sum_{l=1}^{3} \mathbf{c}_{i j k l}(\boldsymbol{\eta}) \frac{\partial \mathbf{G}_{k n}^{\mathbf{w}}(\boldsymbol{\eta}, t-\tau ; \mathbf{r}, 0)}{\partial \boldsymbol{\eta}_{l}} \mathbf{v}_{j}(\boldsymbol{\eta}) d \varsigma^{-}\right\} \\
& +\int_{-\infty}^{\infty} d \tau \int_{S} \sum_{i=1}^{3}\left(\mathbf{G}_{i n}^{\mathbf{w}}(\boldsymbol{\xi}, t-\tau ; \mathbf{r}, 0) \mathbf{t}_{i}(\boldsymbol{\xi}, \mathbf{n}(\boldsymbol{\xi}), \tau, \mathbf{u})\right. \\
& \left.-\mathbf{u}_{i}(\boldsymbol{\xi}, \tau) \sum_{j=1}^{3} \sum_{k=1}^{3} \sum_{l=1}^{3} \mathbf{c}_{i j k l}(\boldsymbol{\xi}) \frac{\partial \mathbf{G}_{k n}^{\mathbf{w}}(\boldsymbol{\xi}, t-\tau ; \mathbf{r}, 0)}{\partial \boldsymbol{\xi}_{l}} \mathbf{n}_{j}(\boldsymbol{\xi})\right) d S .
\end{aligned}
$$

As displacement fields $\mathbf{u}$ and $\mathbf{w}$ satisfy the same homogeneous boundary conditions on $S$, i.e.,

$$
\begin{aligned}
\mathbf{u}(\boldsymbol{\xi}, \tau)=\mathbf{w}(\boldsymbol{\xi}, \tau) & =\mathbf{0} \quad \text { or } \\
\mathbf{t}(\boldsymbol{\xi}, \mathbf{n}(\boldsymbol{\xi}), \tau, \mathbf{u})=\mathbf{t}(\boldsymbol{\xi}, \mathbf{n}(\boldsymbol{\xi}), \tau, \mathbf{w})=\mathbf{0} &
\end{aligned}
$$

at every $\boldsymbol{\xi}$ on surface $S$ throughout all time $\tau \in \mathbb{R}$, we have

$$
\begin{array}{r}
\int_{-\infty}^{\infty} d \tau \int_{S} \sum_{i=1}^{3}\left(\mathbf{G}_{i n}^{\mathbf{w}}(\boldsymbol{\xi}, t-\tau ; \mathbf{r}, 0) \mathbf{t}_{i}(\boldsymbol{\xi}, \mathbf{n}(\boldsymbol{\xi}), \tau, \mathbf{u})\right. \\
\left.-\mathbf{u}_{i}(\boldsymbol{\xi}, \tau) \sum_{j=1}^{3} \sum_{k=1}^{3} \sum_{l=1}^{3} \mathbf{c}_{i j k l}(\boldsymbol{\xi}) \frac{\partial \mathbf{G}_{k n}^{\mathbf{w}}(\boldsymbol{\xi}, t-\tau ; \mathbf{r}, 0)}{\partial \boldsymbol{\xi}_{l}} \mathbf{n}_{j}(\boldsymbol{\xi})\right) d S=0
\end{array}
$$

As Green's tensor $\mathbf{G}^{\mathbf{w}}$ satisfies the equation of motion even on $\varsigma$ (i.e., it and its derivatives are continuous on $\varsigma$ ), we have

$$
\left.\mathbf{G}^{\mathbf{w}}(\boldsymbol{\eta}, t-\tau ; \mathbf{r}, 0)\right|_{\varsigma^{+}}=\left.\mathbf{G}^{\mathbf{w}}(\boldsymbol{\eta}, t-\tau ; \mathbf{r}, 0)\right|_{\varsigma^{-}}
$$

and

$$
\left.\frac{\partial \mathbf{G}_{k n}^{\mathbf{w}}(\boldsymbol{\eta}, t-\tau ; \mathbf{r}, 0)}{\partial \boldsymbol{\eta}_{l}}\right|_{\varsigma^{+}}=\left.\frac{\partial \mathbf{G}_{k n}^{\mathbf{w}}(\boldsymbol{\eta}, t-\tau ; \mathbf{r}, 0)}{\partial \boldsymbol{\eta}_{l}}\right|_{\varsigma^{-}}
$$

for all $k, l, n \in\{1,2,3\}$ and all $\boldsymbol{\eta} \in \varsigma$. The continuity of the Green's tensor $\mathbf{G}^{\mathbf{w}}$ on $\varsigma$ implies that $\mathbf{G}^{\mathbf{w}}$ is continuous throughout the whole medium. Thus, due to the homogeneous boundary conditions of w on $S$, Lemma 12.3 can be 
invoked. With (98), (99), (100) and Lemma 12.3, representation 97) can be rewritten as:

$$
\begin{aligned}
& \mathbf{u}_{n}(\mathbf{r}, t)=\int_{-\infty}^{\infty} d \tau \int_{V} \sum_{i=1}^{3} \mathbf{G}_{i n}^{\mathbf{w}}(\boldsymbol{\xi}, t-\tau ; \mathbf{r}, 0) \mathbf{f}_{i}(\boldsymbol{\xi}, \tau) d V \\
& +\int_{-\infty}^{\infty} d \tau\left\{\int_{\varsigma^{+}} \sum_{i=1}^{3} \mathbf{G}_{i n}^{\mathbf{w}}(\boldsymbol{\eta}, t-\tau ; \mathbf{r}, 0) \mathbf{t}_{i}(\boldsymbol{\eta},-\mathbf{v}(\boldsymbol{\eta}), \tau, \mathbf{u}) d \varsigma^{+}\right. \\
& +\int_{\varsigma^{-}} \sum_{i=1}^{3} \sum_{i=1}^{3} \mathbf{G}_{i n}^{\mathbf{w}}(\boldsymbol{\eta}, t-\tau ; \mathbf{r}, 0) \mathbf{t}_{i}(\boldsymbol{\eta}, \mathbf{v}(\boldsymbol{\eta}), \tau, \mathbf{u}) d \varsigma^{-} \\
& -\int_{\varsigma^{+}} \sum_{i=1}^{3} \mathbf{u}_{i}(\boldsymbol{\eta}, \tau) \sum_{j=1}^{3} \sum_{k=1}^{3} \sum_{l=1}^{3} \mathbf{c}_{i j k l}(\boldsymbol{\eta}) \frac{\partial \mathbf{G}_{k n}^{\mathbf{w}}(\boldsymbol{\eta}, t-\tau ; \mathbf{r}, 0)}{\partial \boldsymbol{\eta}_{l}}\left(-\mathbf{v}_{j}(\boldsymbol{\eta})\right) d \varsigma^{+} \\
& \left.-\int_{\varsigma^{-}} \mathbf{u}_{i}(\boldsymbol{\eta}, \tau) \sum_{j=1}^{3} \sum_{k=1}^{3} \sum_{l=1}^{3} \mathbf{c}_{i j k l}(\boldsymbol{\eta}) \frac{\partial \mathbf{G}_{k n}^{\mathbf{w}}(\boldsymbol{\eta}, t-\tau ; \mathbf{r}, 0)}{\partial \boldsymbol{\eta}_{l}} \mathbf{v}_{j}(\boldsymbol{\eta}) d \varsigma^{-}\right\} \\
& =\int_{-\infty}^{\infty} d \tau \int_{V} \sum_{i=1}^{3} \mathbf{G}_{i n}^{\mathbf{w}}(\boldsymbol{\xi}, t-\tau ; \mathbf{r}, 0) \mathbf{f}_{i}(\boldsymbol{\xi}, \tau) d V \\
& +\int_{-\infty}^{\infty} d \tau\left\{\int_{\varsigma} \sum_{i=1}^{3} \mathbf{G}_{i n}^{\mathbf{w}}(\boldsymbol{\eta}, t-\tau ; \mathbf{r}, 0)\left(\left.\mathbf{t}_{i}(\boldsymbol{\eta},-\mathbf{v}(\boldsymbol{\eta}), \tau, \mathbf{u})\right|_{\varsigma^{+}}+\left.\mathbf{t}_{i}(\boldsymbol{\eta}, \mathbf{v}(\boldsymbol{\eta}), \tau, \mathbf{u})\right|_{\varsigma^{-}}\right) d \varsigma\right. \\
& \left.+\int_{\varsigma} \sum_{i=1}^{3} \Delta \mathbf{u}_{i}(\boldsymbol{\eta}, \tau) \sum_{j=1}^{3} \sum_{k=1}^{3} \sum_{l=1}^{3} \mathbf{c}_{i j k l}(\boldsymbol{\eta}) \frac{\partial \mathbf{G}_{k n}^{\mathbf{w}}(\boldsymbol{\eta}, t-\tau ; \mathbf{r}, 0)}{\partial \boldsymbol{\eta}_{l}} \mathbf{v}_{j}(\boldsymbol{\eta}) d \varsigma\right\} \\
& =\int_{-\infty}^{\infty} d \tau \int_{V} \sum_{i=1}^{3} \mathbf{G}_{n i}^{\mathbf{w}}(\mathbf{r}, t-\tau ; \boldsymbol{\xi}, 0) \mathbf{f}_{i}(\boldsymbol{\xi}, \tau) d V \\
& +\int_{-\infty}^{\infty} d \tau\left\{\int_{\varsigma} \sum_{i=1}^{3} \mathbf{G}_{n i}^{\mathbf{w}}(\mathbf{r}, t-\tau ; \boldsymbol{\eta}, 0)\left(\left.\mathbf{t}_{i}(\boldsymbol{\eta},-\mathbf{v}(\boldsymbol{\eta}), \tau, \mathbf{u})\right|_{\varsigma^{+}}+\left.\mathbf{t}_{i}(\boldsymbol{\eta}, \mathbf{v}(\boldsymbol{\eta}), \tau, \mathbf{u})\right|_{\varsigma^{-}}\right) d \varsigma\right. \\
& \left.+\int_{\varsigma} \sum_{i=1}^{3} \Delta \mathbf{u}_{i}(\boldsymbol{\eta}, \tau) \sum_{j=1}^{3} \sum_{k=1}^{3} \sum_{l=1}^{3} \mathbf{c}_{i j k l}(\boldsymbol{\eta}) \frac{\partial \mathbf{G}_{n k}^{\mathbf{w}}(\mathbf{r}, t-\tau ; \boldsymbol{\eta}, 0)}{\partial \boldsymbol{\eta}_{l}} \mathbf{v}_{j}(\boldsymbol{\eta}) d \varsigma\right\}
\end{aligned}
$$

which proves the theorem.

The theorem 16.1 states that the displacement field $\mathbf{u}$ consists of three components:

- The first term

$$
\int_{-\infty}^{\infty} d \tau \int_{V} \sum_{i=1}^{3} \mathbf{G}_{n i}^{\mathbf{w}}(\mathbf{r}, t-\tau ; \boldsymbol{\xi}, 0) \mathbf{f}_{i}(\boldsymbol{\xi}, \tau) d V
$$

indicates that the displacement field $\mathbf{u}$ is caused by the volume force field $\mathbf{f}$;

- The second term

$$
\int_{-\infty}^{\infty} d \tau \int_{\varsigma} \sum_{i=1}^{3} \mathbf{G}_{n i}^{\mathbf{w}}(\mathbf{r}, t-\tau ; \boldsymbol{\eta}, 0)\left(\left.\mathbf{t}_{i}(\boldsymbol{\eta},-\mathbf{v}(\boldsymbol{\eta}), \tau, \mathbf{u})\right|_{\varsigma^{+}}+\left.\mathbf{t}_{i}(\boldsymbol{\eta}, \mathbf{v}(\boldsymbol{\eta}), \tau, \mathbf{u})\right|_{\varsigma^{-}}\right) d \varsigma
$$

implies that the tractions on $\varsigma$ are the causes for the displacements. The discontinuities of tractions on $\varsigma$,

$$
\left.\mathbf{t}(\boldsymbol{\eta},-\mathbf{v}(\boldsymbol{\eta}), \tau, \mathbf{u})\right|_{\varsigma^{+}}+\left.\mathbf{t}(\boldsymbol{\eta}, \mathbf{v}(\boldsymbol{\eta}), \tau, \mathbf{u})\right|_{\varsigma^{-}},
$$

is also called the stress glut in seismology and is considered as the driver for fault slips;

- The third term

$$
\int_{-\infty}^{\infty} d \tau \int_{\varsigma} \sum_{i=1}^{3} \Delta \mathbf{u}_{i}(\boldsymbol{\eta}, \tau) \sum_{j=1}^{3} \sum_{k=1}^{3} \sum_{l=1}^{3} \mathbf{c}_{i j k l}(\boldsymbol{\eta}) \frac{\partial \mathbf{G}_{n k}^{\mathbf{w}}(\mathbf{r}, t-\tau ; \boldsymbol{\eta}, 0)}{\partial \boldsymbol{\eta}_{l}} \mathbf{v}_{j}(\boldsymbol{\eta}) d \varsigma
$$


says that the displacements are the results of the displacement dislocations (discontinuities) across $\varsigma$. In seismology, this term is a kinematic model as it describes the earthquake displacements as the direct consequences of the relative movements between the two faces of a fault plane. This term is a very important representation in seismology as it presents a straightforward relation between the displacement field and the fault slips without any reference to the stresses that cause the slips.

In this tutorial we focus on the kinematic model, thus in the remainder of this section we show how the representation 96 can be reduced to (101). To achieve this, we need to make two assumptions:

- Assumption 1: The tractions due to displacement $\mathbf{u}$ are continuous across $\varsigma$, i.e.,

$$
\left.\mathbf{t}(\boldsymbol{\eta},-\mathbf{v}(\boldsymbol{\eta}), t, \mathbf{u})\right|_{\varsigma^{+}}+\left.\mathbf{t}(\boldsymbol{\eta}, \mathbf{v}(\boldsymbol{\eta}), t, \mathbf{u})\right|_{\varsigma^{-}}=\mathbf{0}
$$

for all $\boldsymbol{\eta} \in \varsigma$ and all $t \in \mathbb{R}$;

- Assumption 2: The volume force for the displacement field $\mathbf{u}$ is zero throughout the medium and time, i.e.,

$$
\mathbf{f}(\boldsymbol{\xi}, t)=\mathbf{0} \quad \forall \boldsymbol{\xi} \in V \quad \text { and } \quad \forall t \in \mathbb{R} .
$$

Under these two assumptions, the representation $(96)$ is then reduced to the kinematic model (101).

Remark It should be noted that in deriving the theorem 16.1 we choose boundary conditions for $\mathbf{u}$ and $\mathbf{w}$ on $\varsigma$ independently. This is permitted by the Reciprocity Theorem 12.2, which allows for different boundary conditions for $\mathbf{u}$ and $\mathbf{w}$ on $\varsigma$.

\subsection{Representation Theorem with an Internal Surface in Elastostatics}

It is straightforward to show that under the same assumptions made in theorem 16.1 for elastodynamics the representation theorem with an internal surface for elastostatics is given by

$$
\begin{aligned}
\mathbf{u}_{n}(\mathbf{r}) & =\int_{V} \sum_{i=1}^{3} \mathbf{S}_{n i}^{\mathbf{w}}(\mathbf{r} ; \boldsymbol{\xi}) \mathbf{f}_{i}(\boldsymbol{\xi}) d V \\
& +\int_{\varsigma} \sum_{i=1}^{3} \mathbf{S}_{n i}^{\mathbf{w}}(\mathbf{r} ; \boldsymbol{\eta})\left(\left.\mathbf{t}_{i}(\boldsymbol{\eta},-\mathbf{v}(\boldsymbol{\eta}), \mathbf{u})\right|_{\varsigma^{+}}+\left.\mathbf{t}_{i}(\boldsymbol{\eta}, \mathbf{v}(\boldsymbol{\eta}), \mathbf{u})\right|_{\varsigma^{-}}\right) d \varsigma \\
& +\int_{\varsigma} \sum_{i=1}^{3} \Delta \mathbf{u}_{i}(\boldsymbol{\eta}) \sum_{j=1}^{3} \sum_{k=1}^{3} \sum_{l=1}^{3} \mathbf{c}_{i j k l}(\boldsymbol{\eta}) \frac{\partial \mathbf{S}_{n k}^{\mathbf{w}}(\mathbf{r} ; \boldsymbol{\eta})}{\partial \boldsymbol{\eta}_{l}} \mathbf{v}_{j}(\boldsymbol{\eta}) d \varsigma
\end{aligned}
$$

Similarly, we can make assumptions on the traction continuity and zero volume force to obtain the kinematic model in elastostatics:

$$
\mathbf{u}_{n}(\mathbf{r})=\int_{\varsigma} \sum_{i=1}^{3} \Delta \mathbf{u}_{i}(\boldsymbol{\eta}) \sum_{j=1}^{3} \sum_{k=1}^{3} \sum_{l=1}^{3} \mathbf{c}_{i j k l}(\boldsymbol{\eta}) \frac{\partial \mathbf{S}_{n k}^{\mathbf{w}}(\mathbf{r} ; \boldsymbol{\eta})}{\partial \boldsymbol{\eta}_{l}} \mathbf{v}_{j}(\boldsymbol{\eta}) d \varsigma
$$

Particularly, if the medium is isotropic we can plug equation (9) into (103) and obtain

$$
\mathbf{u}_{n}(\mathbf{r})=\int \sum_{\varsigma}^{3} \Delta \mathbf{u}_{i}(\boldsymbol{\eta}) \sum_{j=1}^{3}\left\{\lambda(\boldsymbol{\eta}) \delta_{i j} \sum_{k=1}^{3} \frac{\partial \mathbf{S}_{n k}^{\mathbf{w}}(\mathbf{r} ; \boldsymbol{\eta})}{\partial \boldsymbol{\eta}_{k}}+\mu(\boldsymbol{\eta})\left(\frac{\partial \mathbf{S}_{n i}^{\mathbf{w}}(\mathbf{r} ; \boldsymbol{\eta})}{\partial \boldsymbol{\eta}_{j}}+\frac{\partial \mathbf{S}_{n j}^{\mathbf{w}}(\mathbf{r} ; \boldsymbol{\eta})}{\partial \boldsymbol{\eta}_{i}}\right)\right\} \mathbf{v}_{j}(\boldsymbol{\eta}) d \varsigma
$$

Furthermore, if the medium is homogeneous we have from (104):

$$
\mathbf{u}_{n}(\mathbf{r})=\int_{\varsigma} \sum_{i=1}^{3} \Delta \mathbf{u}_{i}(\boldsymbol{\eta}) \sum_{j=1}^{3}\left\{\lambda \delta_{i j} \sum_{k=1}^{3} \frac{\partial \mathbf{S}_{n k}^{\mathbf{w}}(\mathbf{r} ; \boldsymbol{\eta})}{\partial \boldsymbol{\eta}_{k}}+\mu\left(\frac{\partial \mathbf{S}_{n i}^{\mathbf{w}}(\mathbf{r} ; \boldsymbol{\eta})}{\partial \boldsymbol{\eta}_{j}}+\frac{\partial \mathbf{S}_{n j}^{\mathbf{w}}(\mathbf{r} ; \boldsymbol{\eta})}{\partial \boldsymbol{\eta}_{i}}\right)\right\} \mathbf{v}_{j}(\boldsymbol{\eta}) d \varsigma
$$

which is the famous Steketee's formula (Steketee 1958). 


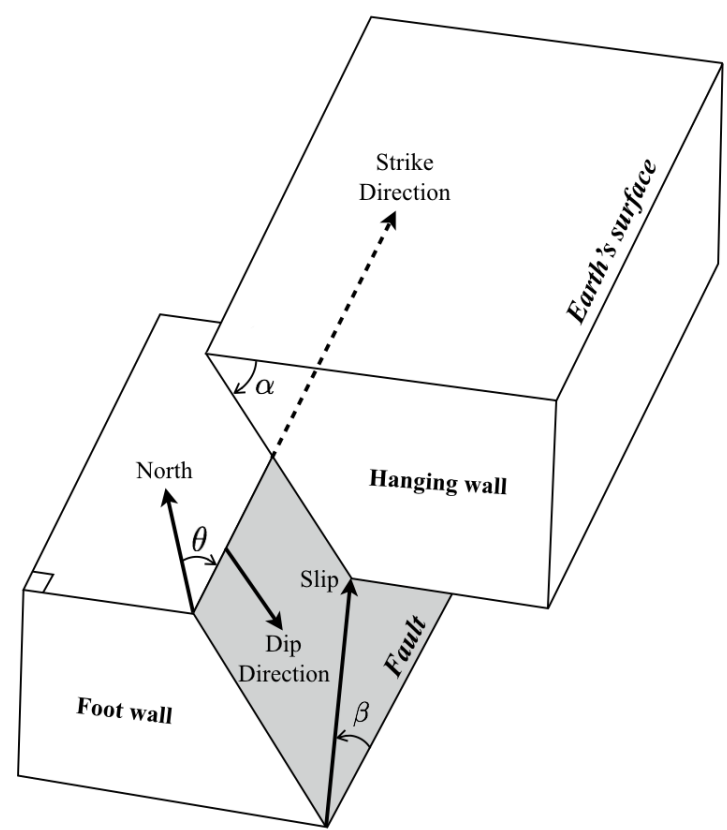

Figure 3: The geometry of a faulting: The grey area is the fault surface; $\alpha$ is the dip; $\beta$ is the strike; $\theta$ is the rake.

\section{Faulting Geometry}

The geometry of an earthquake faulting (Figure 3) can be described by three angular measurements (strike, dip and rake) and the magnitude of the slip.

Dip and dip direction. Dip ( $\alpha$ in Figure 3 ) is the angle used to describe the steepness of a fault surface. The angle is between $0^{\circ}$ and $90^{\circ}$ and is measured from Earth's surface, or a plane parallel to Earth's surface, to the fault surface. The dip direction is the direction toward which the fault surface is inclined.A fault with dip of $0^{\circ}$ is called horizontal fault while a fault with dip of $90^{\circ}$ is called vertical fault.

Foot wall and hanging wall. For non-vertical faults, the foot wall is the lower fault block beneath Earth's surface and the fault surface (grey area in Figure 33, while the hanging wall is the upper fault block that is beneath Earth's surface and above the fault surface. For vertical fault, the foot wall is assumed to be on the left of an observer looking in the strike direction.

Strike and strike direction. The strike $\left(\theta\right.$ in Figure 3 is the angle between $0^{\circ}$ and $360^{\circ}$ used to specify the orientation of a fault. To determine the strike, strike direction needs to be decided first. The strike direction is the direction an observer looks along the fault line (i.e., the intersection of Earth's surface and the fault surface) when he stands on the Earth's surface with the foot wall on his left while the hanging wall on his right. The strike is then measured clockwise from North direction to the strike direction.

Slip and rake. The slip is a parameter used to describe the motion of a fault. The slip is a vector, meaning that it has magnitude and direction. The magnitude of a slip is simply the distance that a hanging wall moves relative to the foot wall. The direction of slip is the direction that hanging wall moves relative to the foot wall. The rake ( $\beta$ in Figure 3 is the angle between $0^{\circ}$ and $360^{\circ}$ measured anticlockwise from the strike direction to the slip direction. According to the slip direction, the faulting can be classified into two types termed strike-slip and dip-slip. The faulting is strike-slip if the slip direction is parallel to the strike direction (i.e., the rake $\beta=0^{\circ}$ or $\beta=180^{\circ}$ ); the fault is dip-slip if the 

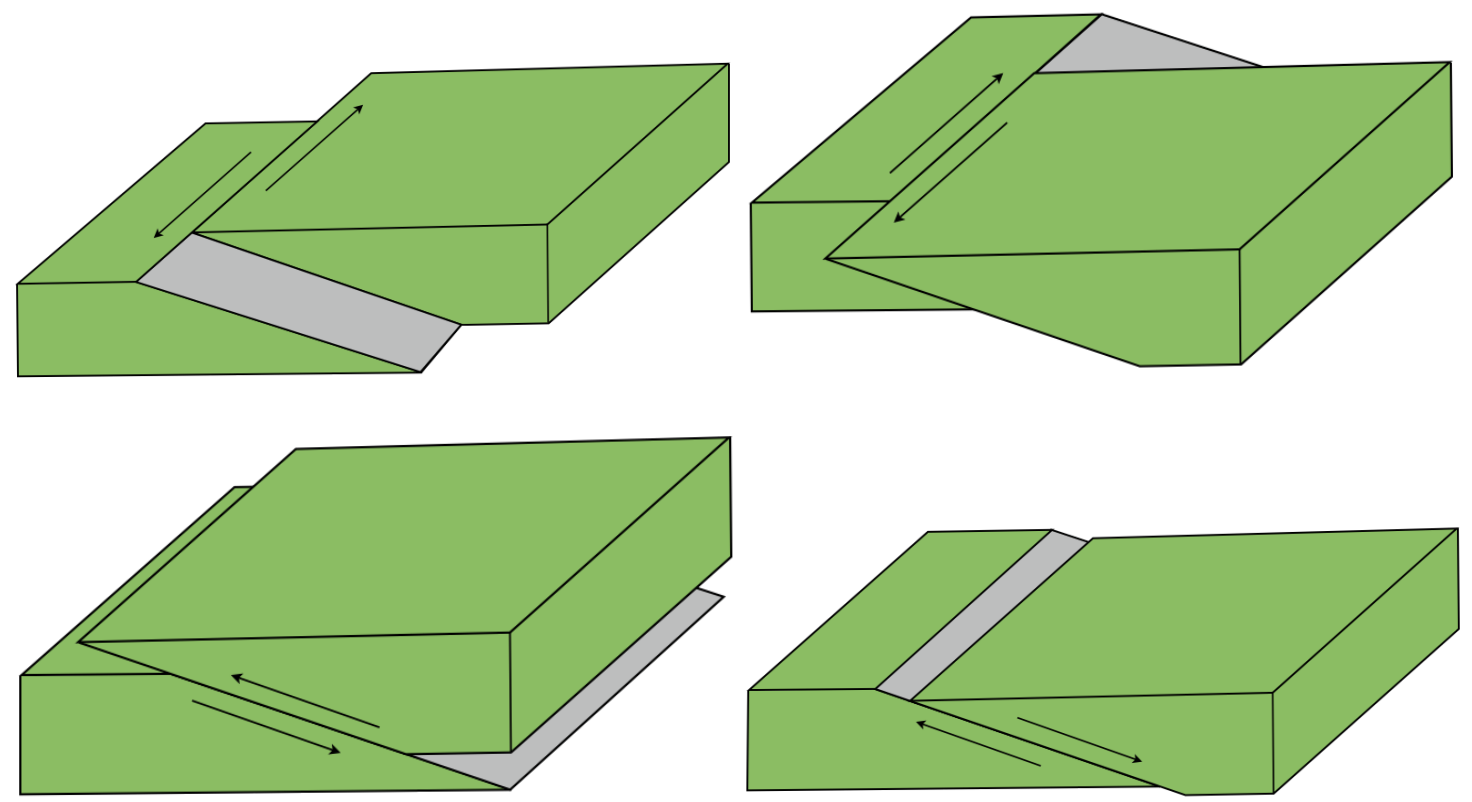

Figure 4: (Top-left) The left-lateral strike-slip faulting. (Top-right) The right-lateral strike-slip faulting. (Bottom-left) The reverse dip-slip faulting. (Bottom-right) The normal dip-slip faulting.

slip direction is perpendicular to the strike direction (i.e., the rake $\beta=90^{\circ}$ or $\beta=270^{\circ}$ ). The strike-slip and dip-slip faulting can be further categorised:

- Strike-slip: If an observer, standing on one side of a fault, finds that the adjunct side moves to the left, then the faulting is left-lateral strike-slip (i.e., slip has the same direction with the strike direction or the rake $\beta=0^{\circ}$ ). If the adjunct side moves to the right, then the faulting is right-lateral strike-slip (i.e., slip has the opposite direction with the strike direction or the rake $\beta=180^{\circ}$ ).

- Dip-slip: If the hanging wall moves upward relative to the foot wall (i.e., $\beta=90^{\circ}$ ), the faulting is termed reverse, whereas the hanging wall moves downward relative to the foot wall (i.e., $\beta=270^{\circ}$ ), the faulting is called normal.

Figure 4 illustrates the faulting types explained. There are some unusual faulting types such as tensile faulting that not only includes strike- and dip-slips but also have expansion and compression of faults.

\section{References}

Aki, K. (2009), Quantitative Seismology, 2nd edn, University Science Books, Sausalito, CA.

Bower, A. (2005), '3.2 Singular Solutions for an Infinite Solid', Lecture notes for EN224-Linear Elasticity. Available at: https://www.brown.edu/Departments/Engineering/Courses/EN224/notes.htm

Chau, K. (2012), Analytic Methods in Geomechanics, CRC Press, pp. 122-128.

Irgens, F. (2008), Continuum Mechanics, Springer Science \& Business Media.

Kachanov, M. L., Shafiro, B. \& Tsukrov, I. (2013), Handbook of Elasticity Solutions, Springer Science \& Business Media, pp. 36-37.

Kelvin, L. (1848), 'Note on the integration of the equations of equilibrium of an elastic solid', Cambridge and Dublin Mathematical Journal 3, 87-89. 
Lay, T. \& Wallace, T. C. (1995), Modern Global Seismology, Vol. 58, Academic press, pp. 323-325.

Matthews, P. C. (2012), Vector Calculus, Springer Science \& Business Media.

Mindlin, R. (1953), Force at a point in the interior of a semi-infinite solid, Technical report, DTIC Document.

Mindlin, R. D. (1936), 'Force at a point in the interior of a semi-infinite solid', Physics 7(5), 195-202.

Mindlin, R. D. \& Cheng, D. H. (1950), 'Nuclei of strain in the semi-infinite solid', Journal of Applied Physics 21(9), 926-930.

Moczo, P. (2006), 'Lecture Notes: Introduction to Theoretical Seismology'. Available at: http://www . earthphysics. sk/mainpage/stud_mat/Introduction_to_Theoretical_Seismology.pdf

Okada, Y. (1992), 'Internal deformation due to shear and tensile faults in a half-space', Bulletin of the Seismological Society of America 82(2), 1018-1040.

Press, F. (1965), 'Displacements, strains, and tilts at teleseismic distances', Journal of Geophysical Research 70(10), 2395-2412.

Robinson, R. \& Benites, R. (1996), 'Synthetic seismicity models for the Wellington region, New Zealand: Implications for the temporal distribution of large events', Journal of Geophysical Research: Solid Earth 101(B12), $27833-27844$.

Shearer, P. M. (2009), Introduction to Seismology, Cambridge University Press.

Steketee, J. (1958), 'On Volterra's dislocations in a semi-infinite elastic medium', Canadian Journal of Physics 36(2), 192-205.

Sternberg, E. (1980), On singular problems in linearized and finite elastostatics., Technical report, DTIC Document.

Udías, A., Madariaga, R. \& Buforn, E. (2014), Source Mechanisms of Earthquakes: Theory and Practice, Cambridge University Press.

Zhou, S., Johnston, S., Robinson, R. \& Vere-Jones, D. (2006), 'Tests of the precursory accelerating moment release model using a synthetic seismicity model for Wellington, New Zealand', Journal of Geophysical Research: Solid Earth 111(B5). 